\title{
DAVID WINDRIDGE
}

\author{
Department of Electronic Engineering, \\ University of Surrey, Guildford, \\ Surrey, GU2 7XH, \\ United Kingdom. \\ D.Windridge@surrey .ac.uk
}

\begin{abstract}
We set out in this review article to construct a generalized theory of classifier combination for classifiers that, at least in the theory's initial form, act within noncoincident feature-spaces. Doing so involves the postulation of an equivalence between the various strategies for classifier combination and the tomographic reconstruction of the joint pattern-space probability density function, where the classifiers themselves are interpreted as extremely bandwidth limited Radon transform data. This analogue will immediately suggest techniques for improving the process, as well as defining the optimal performance to be gained by such combinatorial approaches with respect to arbitrary joint pattern-space PDF morphologies. Furthermore, this methodology of optimality naturally will also encompass the feature selection process to present a unified perspective on the various differing aspects of classifier combination.
\end{abstract}

A practical implementation of the methodology is also given, along with a series of tests to establish its performance in relation to both model and real-word classification scenarios. 


\section{Introduction}

\subsection{Construction of a Generalized Theory of Classifier Fusion}

The limitation on the performance gains that can be derived from a single classifier is a reflection of the fact that any individual design must, in the absence of an exhaustive training set, necessarily encode an a priori, meta-statistical assumption as to the type of morphology applicable to the decision boundary: the potentially infinite variety of real-world data would seem to indicate, however, that no such singular assumption could ever be fully justified:

" No single model exists for all pattern recognition problems and no single technique is applicable to all problems. Rather what we have is a bag of tools and a bag of problems." (Kanal 1974)

This observation has engendered considerable recent interest in Multiple Classifier Systems $[1,2,3,4,5,6]$, which seek to make use of the divergence in design methodologies to limit such a priori impositions and obtain a correspondingly better estimate of the decision boundary in order to boost classification performance.

In seeking to establish a general theoretical framework for such approaches, we will attempt to determine that classifier combination in virtually all of its variant forms has an aspect which may be regarded as an approximate attempt at the reconstruction of the combined pattern space by tomographic means, the feature selection process in this scenario constituting an implicit Radon integration along the lines of the physical processes involved in CAT scanning, etc, albeit of a multi-dimensional nature. (An indication of precisely what we envisage by this equivalence between Radon integration 
and feature-selection is given in Figure 1 for the two-dimensional case). It will thereby be ascertained that a morphologically optimal strategy for classifier combination can be achieved by appropriately restructuring the feature-selection algorithm such that a fully-constituted tomographic combination (rather than the approximation) acts in its most appropriate domain: that is, when the combination is comprised of classifiers with distinct feature sets.

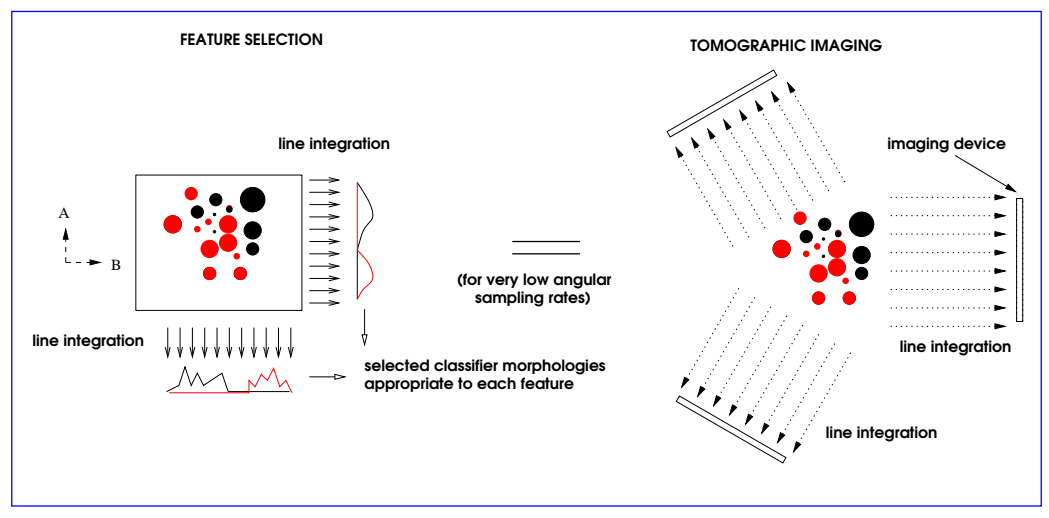

Figure 1: Radon integration

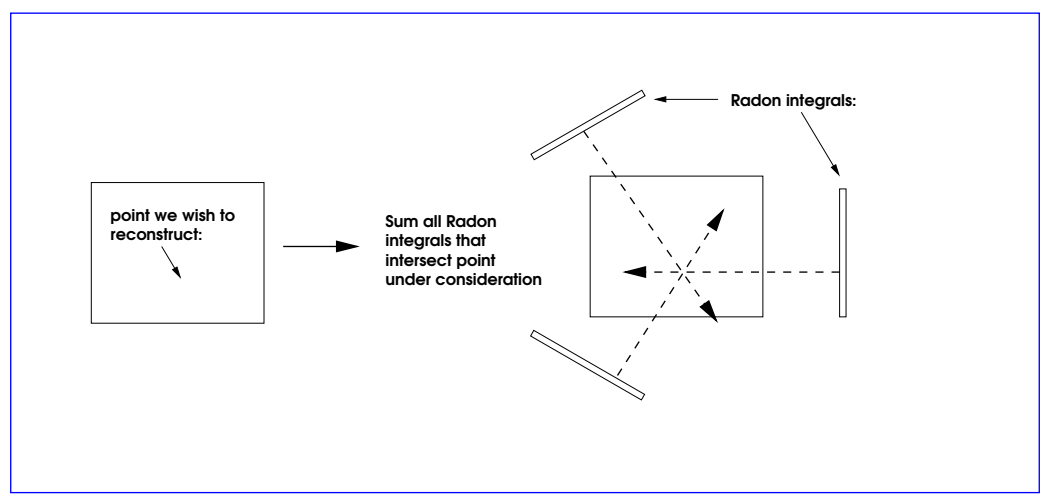

Figure 2: Back-projection

As in medical imaging, this fully constituted tomographic combination necessarily involves the application of a deconvolution procedure to a back-projected space (cf fig 2 ), which, in the context of pattern-recognition, we will demonstrate amounts to the composite probability density function (PDF) constructed implicitly by the Sum-Rule 
decision scheme (eg [2]). In conventional implementations of tomography [8], such deconvolution is most usually accomplished via a collective prior filtering of the Radon integrals in order to remove reconstruction artifacts (as illustrated in figures 3 and 4). This would typically take the form of a differentiation operator that acts to remove what, in the reconstructive space, would (for the case of perfect angular sample coverage) amount to convolution by an $|1 / r|$ function.

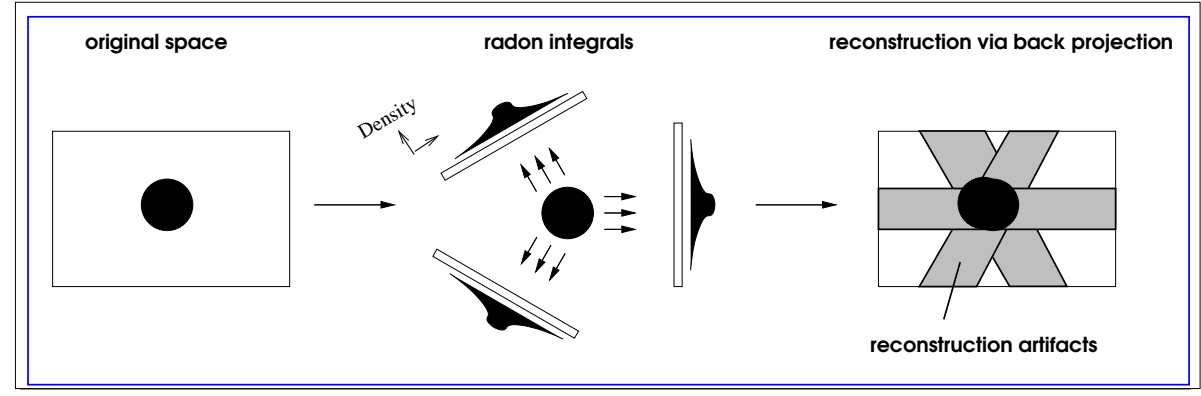

Figure 3: Reconstruction artifacts in the back projected space

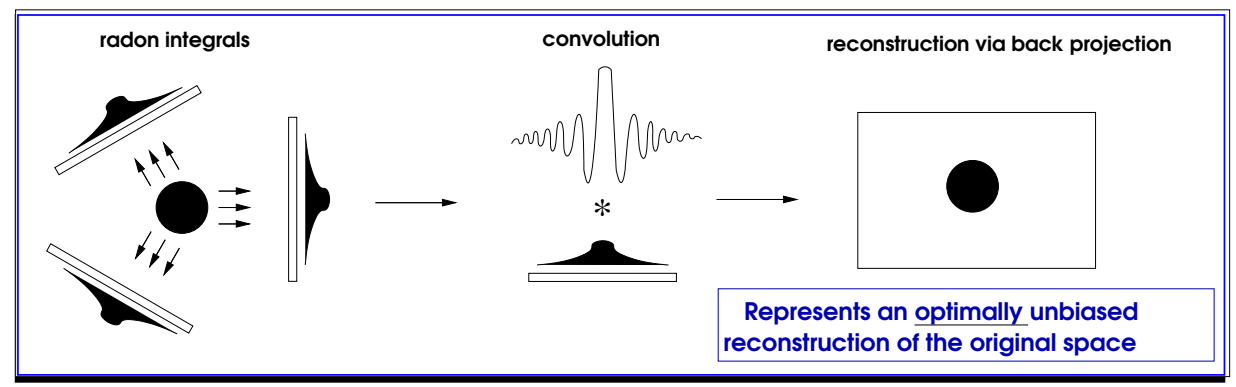

Figure 4: Back-projection with prior filtration of Radon integrals

The very low angular sampling implied by feature-selection, however, as well as the dimensionality of the spaces involved, means that an appropriate form of tomography theory needs to be developed from first principles. This, and the testing of the methodology so derived will be the endeavor of the following article, which, as such, constitutes a review of, and an expansion upon, the author's existing work in the field, in particular [17] (in respect to which the publishers retain copyright over certain diagrams 
and derivations employed throughout the following, to be indicated at the appropriate points).

\subsection{Existing Approaches to Classifier Combination}

Our investigation commences with a brief overview of the various pre-existing methods of classifier fusion. We have indicated that the non-overlapping of the misclassification errors of very distinct methods of classification has lead to the realization that, in general, no one method of classification can circumscribe all aspects of a typical realworld classification problem, prompting, in consequence, the investigation of a variety of combinatorial methods in a bid to improve classification performance. Generally, these methods have in common, at least in regard to the final combination, that they are based on intuitive techniques for the combination of disparate decision schemes (eg majority vote, weighted mean), and not upon any underlying theoretical schematics. In particular, there has not as yet been any attempt to obtain a generally optimal mathematical solution to the problem on meta-statistical grounds.

This fact, however, has not prevented a large body of effective, heuristically-conceived techniques being incorporated into the machine learning toolkit. The most notably successful such approaches divide into two principle areas of concentration: decision fusion and ensemble creation. In the former, we are concerned only with obtaining an overall class decision from the various classifier outputs, working either with hard decisions (eg maximum vote), or else probabilistic outputs (sum rule, product rule etc). In the latter, however, we are principally concerned with mechanisms for either diversifying, or else increasing the representative capacity of the classifiers constituting the combination. This

approach further divides into two sub-categories: subspace methods ([26]) and training 
set perturbation techniques (boosting [25] and bootstrap aggregation; 'bagging' [24]).

The latter two techniques have, in particular, achieved widespread successes, and can be seen as methods for tuning a given base classifier's bias or variance, respectively [27].

Our concern, however, is ostensibly only with decision fusion and its optimization (as opposed to ensemble creation), although the later theoretical necessity of delineating combination types on the basis of whether the constituent classifiers share a common feature-space means that ensemble methods are implicitly considered in our proposed framework for classifier fusion. Given that we may also diversify classifier morphology via existing combination methodologies, we are hence, in this article, effectively proposing a meta-combination strategy capable of incorporating all existing work in the field within a common framework, albeit at an implicit level: section 2.7 addresses this point in more detail.

\subsubsection{Issue of Feature-Selection}

Classifier combination almost always takes place in the context of feature-selection either implicitly or explicitly. That is, features are either pre-correlated with classifiers on and a priori (possibly physically motivated) basis, or else allocated by a specific featureselection process (such as forward searching) acting via a performance-based criterion. The actual number of features allocated to each classifier depends on the outcome of a tension between the two opposing constraints of, firstly, more accurate parameter determination through increased sampling rates, and secondly, maximized retention of morphological information through increased dimensionality. These contrary considerations are summarized in figure 5.

We shall argue later in the article that any selected mechanism for decision fusion acts 


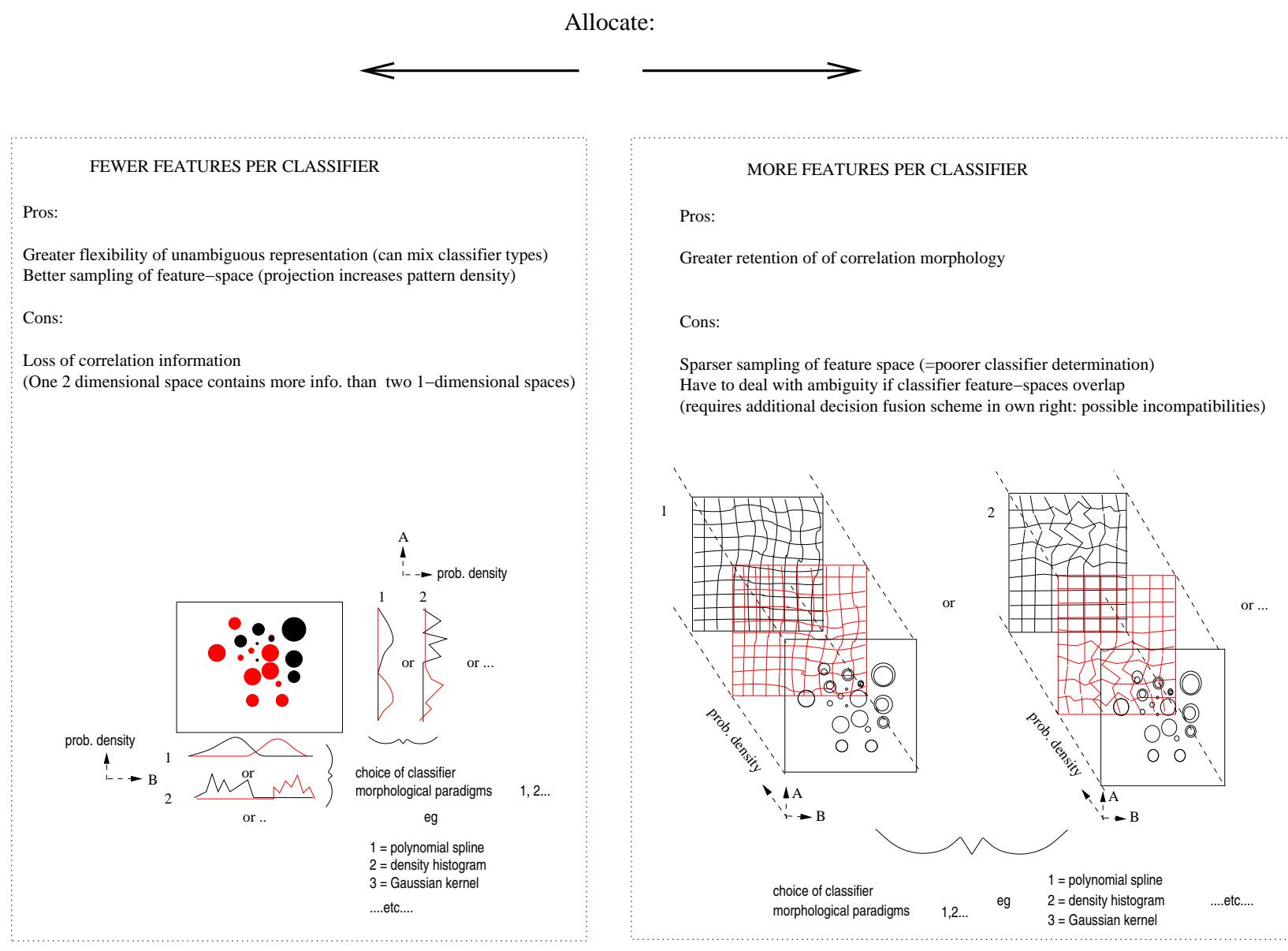

Figure 5:

fundamentally differently for classifiers that act on common feature spaces in relation to those that act on discrete sets of feature-spaces, and that this issue needs to be addressed at the feature selection level in order to avoid the systemic ambiguities that have hitherto been involved (if generally ignored). Ultimately, we shall discover that optimality requires that we abandon altogether the notion of a feature-selection process distinct from classifier combination, and consider instead a hybrid process. 


\subsection{Tomography Theory and its Applications}

Tomography theory, upon which our work will be constructed, is a well developed branch of applied mathematics that finds its chief application in the area of image reconstruction. Typically, an imaging modality measures a series of intersecting slices (Greek: 'tomos') of a two or three dimensional manifold containing volumetric (or areal) information. Constraint information for an individual point-value in this space is thus spread over a number of individual slices, and it is the task of tomographic reconstruction to invert this process and determine, as far as possible, the aggregate point values of the measured space. Very often this problem is ill-posed, and an a priori supposition as to the nature of the underlying morphology must be made in order to arrive at a single well-defined solution. Generally, these constraints will be based on reasonable assumption as to the underlying material disposition of the imaged object.

Tomographic reconstruction is thus principally employed where direct, invasive imaging of the object under consideration is either hazardous (medical imaging), unfeasible (seismological earth imaging, stellar imaging), or empirically compromising (plasma imaging). The examples listed give an idea of the scope and extent of the use of tomographic methods in the research arena, with the former, medical imaging, constituting perhaps the single most common practical use of the technique. The imaging modalities themselves exhibit a similar breadth of scope; within the subject medical imaging, a number of different sub-divisions may be made on the basis of the specific data-capturing modality, for instance: 3d ultrasound, CAT scanning, positron emission tomography, and electrical impedance tomography. As long as the constraint of non-invasiveness is an experimental requirement, tomographic methods will continue to enjoy a wide, and potentially ever-expanding range of uses and implementations. Very often such new 
methods will require new a priori constraints, and even novel mathematics, in order to solve the inverse problem presented by the reconstruction of individual points from tomographically-obtained data (see, for instance, [8] for a summary).

Our particular employment of tomographic reconstruction is certainly consistent with this trend, taking place, as it does, in an entirely abstract space (and, moreover, one of arbitrary dimensionality). Consequently, it presents a number of problematic aspects entirely unique in tomography theory and will require the use of a number of novel mathematical devices in order to set unique constraints on the reconstructed probability space.

\subsection{Article Structure}

Given the scope of our inquiry, the format of this article will divide naturally into a number of sub-investigations, the initial sections being concerned with outlining tomographic reconstruction theory and its generalization to the higher-dimensionality, low angular sample-rate pattern spaces appropriate to pattern recognition theory. The immediately following sections will then concern themselves with making the parallels with probability theory mathematically rigorous, and finally considering the generalization of the technique to the combination of classifiers with non-distinct feature sets, and hence the universal application of the method. With the theoretical aspects of the method thus elucidated, we shall instigate a program of investigation into the practical utility of the method, first setting out an economized approach to practical implementation of the methodology, and introducing an intuitive 'iterative graphical correlation' explication of the tomographic method, laying bare the method by which it interrelates constituent classifier morphologies in the combination. We then proceed with a two dimensional 
illustrative example. Following, this we shall set out on a more rigorous program of performance investigation, focusing in particular on the performance trends over increasing classifier dimensionalities, and hence, in conclusion, confirming the method's general applicability to the field of pattern recognition.

\subsubsection{Philosophical Development}

We have argued that the elucidation of this methodology over the course of this article will strongly suggest a very much more unified approach to feature selection in the context of classifier combination, one in which the two apparently distinct processes become inseparable on attempting to obtain the optimal classification performance from a given set of classifiers. This unification comes about quite naturally, through having necessarily made an explicit distinction between the two separate aspects of classifier combination that become apparent; namely, classifier combination as a method of implicitly refining the individual class PDFs, and classifier combination as implicit n-dimensional tomographic reconstruction. The former aspect, the refining of the PDF morphology through combination, may, within the wider theoretical context imposed on us by our methodology, then be treated as a separate classification scheme in its own right. Hence, our investigation gives rise to the notion that classifier combination, in its most rigorous sense, can only apply to those feature sets explicitly selected by the feature selection algorithm to be distinct: that is; distributed over the range of possible classifiers in a non-overlapping manner, and thus combining in a entirely tomographically-reconstructive fashion. The modification of the feature selection algorithm implied by the method therefore involves treating combinations of the classifiers (via any of the preexisting non-tomographic schemes for combination) on exactly the 
same footing as their constituent classifiers within the selection algorithm.

The wider perspective thus evolved in the course of this article would then account for the previous qualitative, but widely observed property of classifier combination, that such methods are especially effective if individual classifiers employ distinct features (see for example $[11,12,13])$, since we may now regard classical combination methods as, to a large degree, implicit, if only partial, tomographic reconstruction algorithms, which are therefore, without prior modification of the feature selection algorithm, attempting to inappropriately conflate the two distinct aspects of combination within the same procedure, unless there exists either a mutual exclusivity or complete identity among the feature sets. We illustrate this point in figure 6 .

The first step in elucidating this generalized view of decision fusion and feature selection is thus the derivation of tomography theory as it applies within the context of classifier fusion, and as such will constitute the exclusive concern of the following section. Much of the methodological development of section 2.1 is quoted directly from [17] (with permission), and is hence the copyright of the IEEE (and similarly, the example and later diagrams of section 4).

\section{Methodology of Tomographic Classifier Fusion}

\subsection{Outline of the Methodology}

In formalizing the framework of this analysis ${ }^{1}$ we shall commence by specifying our prior assumptions as follows (generalizing later to a less constricting set of assumptions):

\footnotetext{
${ }^{1}$ C)2003 IEEE. Reprinted, with permission, from IEEE PAMI, Vol 25, no. 3, March 2003
} 


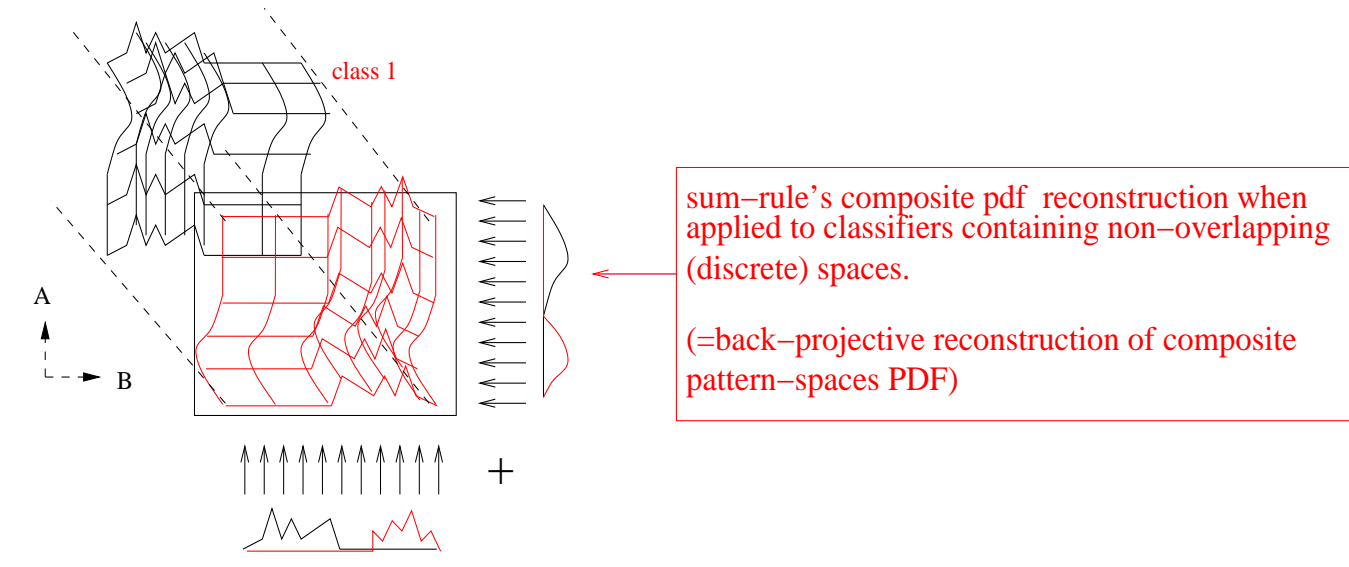

\section{That which is essentially morphological refinement of posterior PDF (ie applied to fully overlapping feature-spaces):}

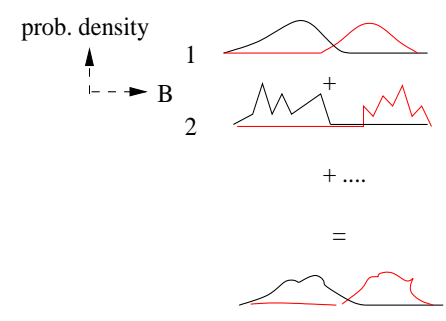

sum-rule reconstruction when applied to classifiers containing overlapping (non-discrete spaces) (=morphological refinement of posterior PDFs

averaged posterior PDF (has combined morphological flexibility approaching the sum of its constituents)

Figure 6: The two distinct aspects of classifier combination

1. We shall assume, at least initially, that the selection of features is decided through classifier preference, and that this is accomplished via the straight-forward omission of superfluous dimensions as appropriate. (note that this will in general differ from the more usual techniques of feature selection, in that we are feature selecting on a class-by-class basis, the separate feature sets for each class only combining at the stage of Bayesian decision making).

2. For simplicity, it shall (at least at the outset) be assumed that the set of classifiers operate on only one feature individually, and that these are distinct (though note 
that the former is not a prerequisite of the method). Evidence that the stronger of these two assumptions, the latter, is reasonably representative of the usual situation comes from [11], wherein features selected within a combinatorial context are consistently shown to favor the allocation of distinct feature sets among the constituent classifiers, presumably due to their divergent design philosophies: the wider implications of the alternative to this assumption are dealt with in section 2.6 .

3. We shall consider that the construction of a classifier is the equivalent of estimating the PDFs $p\left(x_{\mathcal{N}(1, i)}, x_{\mathcal{N}(2, i)} \ldots x_{\mathcal{N}\left(k_{i}, i\right)} \mid \omega_{i}\right) \quad \forall i$, where $\mathcal{N}(x, y)$ is the final set of feature dimensions passed from the feature selection algorithm for class $y$ (the cardinality of which, $k_{i}$, we will initially set to unity for every class identified by the feature selector: ie $\left.k_{i}=1 \forall i\right)$.

4. It is assumed (prior to setting out the feature selection algorithm most appropriate to our technique) that, in any reasonable feature selection regime, the total set of features employed by the various classifiers exhausts the classification information available in the pattern space (ie, the remaining dimensions contribute only a stochastic noise component to the individual clusters).

Given assumption 3 above (that individual classifiers may be regarded as PDFs) and further, that pattern vectors corresponding to a particular class may be regarded as deriving from an $n$-dimensional probability distribution, then the process of feature selection may be envisaged as an integration over the dimensions redundant to that particular classification scheme (the discarding of superfluous dimensions being, in effect, the linear projection of a higher dimensional space onto a lower one, ultimately a 1- 
dimensional space in the above framework). That is, for $n$-dimensional pattern data of class $i$ :

$$
p\left(x_{k} \mid \omega_{i}\right) d x_{k}=\int_{-\infty}^{+\infty} \underbrace{\ldots}_{n-1} \int_{-\infty}^{+\infty} p\left(\vec{X} \mid \omega_{i}\right) d x_{1} \ldots d x_{k-1} d x_{k+1} \ldots d x_{n} . d x_{k}
$$

with $\vec{X}=\left(x_{1}, x_{2}, \ldots, x_{n}\right)$

Because of condition 4 above (a good approximation when a range of classifiers is assumed), we shall consider that the pattern vector effectively terminates at index $j$, where $j \leq n$ is the total number of features (and also classifiers, given condition 3 ). That is, $\vec{X}=\left(x_{1}, x_{2}, \ldots, x_{j}\right)$ now represents the extent of the pattern vector dimensionality. In the integral analogy, the remaining dimensions that are integrated over in equation 1 serve to reduce the stochastic component of the joint PDF by virtue of the increased bin count attributable to each of the pattern vector indices.

Now, it is the basis of our thesis that we may regard equation 1 as the $j$-dimension analogue of the Radon transform (essentially the mathematical equivalent of the physical measurements taken within a tomographic imaging regime), an assertion that we shall make explicit in section 2.4 after having found a method for extending the inverse Radon transform to an arbitrarily large dimensionality. The conventional Radon transform, however, is defined in terms of the two-dimensional function $f(x, y)$ as follows;

$$
R(s, \theta)[f(x, y)]=\int_{-\infty}^{+\infty} \int_{-\infty}^{+\infty} f(x, y) \delta(s-x \cos \theta-y \sin \theta) d x d y(=g(s, \theta))
$$

where $s$ may be regarded as a perpendicular distance to a line in $(\mathrm{x}, \mathrm{y})$ space, and $\theta$ the angle that that line subtends in relation to the $x$ axis. $R(s, \theta)$ is then an integral over 
$f(x, y)$ along the line specified.

As a first approximation to inverting the Radon transform and reconstructing the original data $f(x, y)$, we might apply the Hilbert Space adjoint operator of $R(s, \theta)$, the so-called back-projection operator:

$$
R^{*}[R(s, \theta)](\vec{x})=\int_{\mathcal{S}} R(\vec{\theta}, \vec{\theta} \cdot \vec{x}) d \theta
$$

with $\vec{x}=(x, y), \vec{\theta}=(\cos \theta, \sin \theta)$, and $\mathcal{S}$ the angular extent of the plane of rotation of $\theta$.

To appreciate how this operates, consider first the following identity written in terms of the arbitrary function $v$, where $V=R^{*} v$ :

$$
\begin{aligned}
\int_{\mathcal{S}} \int_{s} v(\theta, \vec{x} \cdot \vec{\theta}-s) g(\theta, s) d s d \theta & =\int_{\mathcal{S}} \int_{s} v(\theta, \vec{x} \cdot \vec{\theta}-s) \int_{\mathcal{R}^{\prime 2}} f\left(\vec{x}^{\prime}\right) \delta\left(s-\vec{x}^{\prime} \cdot \vec{\theta}\right) d^{2} x^{\prime} d s d \theta \\
& \left.=\int_{\mathcal{S}} \int_{R^{\prime 2}} v\left(\theta, \vec{x} \cdot \vec{\theta}-\vec{x}^{\prime} \cdot \vec{\theta}\right) f\left(\vec{x}^{\prime}\right) d^{2} x^{\prime} d \theta \text { (eliminating } s\right) \\
& =\int_{\mathcal{R}^{\prime 2}}\left[\int_{\mathcal{S}} v\left(\theta,\left(\vec{x}-\vec{x}^{\prime}\right) \cdot \vec{\theta}\right) d \theta\right] f\left(\vec{x}^{\prime}\right) d^{2} x^{\prime} \\
& =\int_{\mathcal{R}^{\prime 2}} V\left(\vec{x}-\vec{x}^{\prime}\right) f\left(\vec{x}^{\prime}\right) d^{2} x^{\prime} \quad\left(\text { via the definition of } \mathrm{V}\left[=\mathrm{R}^{*} \mathrm{v}\right]\right) \\
& =V \star f
\end{aligned}
$$

The first term in the above may be symbolically written $R^{*}(v \star g)$, where it is understood that the convolution is with respect to the length variable and not the angular term in $g$. Hence, we have that $V \star f=R^{*}(v \star g)$.

We may also describe the relationship between $V$ and $v$ in terms of their Fourier transforms. Consider first the two-dimensional transform of $V$ :

$$
F(\vec{k})[V(\vec{x})]=(2 \pi)^{-1} \int_{\mathcal{R}^{2}} e^{-i \vec{x} \cdot \vec{k}} V(\vec{x}) d^{2} \vec{x}
$$




$$
\begin{aligned}
& =(2 \pi)^{-1} \int_{\mathcal{R}^{2}} e^{-i \vec{x} \cdot \vec{k}} \int_{\mathcal{S}} v(\theta, \vec{x} \cdot \vec{\theta}) d^{2} x d \theta \quad \text { (by substitution) } \\
& =(2 \pi)^{-1} \int_{\mathcal{S}} \int_{\mathcal{R}^{2}} e^{-i \vec{x} \cdot \vec{k}} v(\theta, \vec{x} \cdot \vec{\theta}) d^{2} x d \theta
\end{aligned}
$$

We now consider a slice through this transform along the direction $\theta$. This may be accomplished in the above by substituting in the delta function $\delta(\vec{k}-\sigma \vec{\theta})$ within the $\theta$ integral (ie coupling the variables $\vec{k}$ and $\vec{\theta}$ ) and transforming it to a $k$ space integral via the corresponding transformation $d \vec{k} \rightarrow \sigma d \vec{\theta}$ ( $\sigma$ is a positive real number) :

$$
\begin{aligned}
F(\sigma \vec{\theta})[V(\vec{x})] & =(2 \pi)^{-1} \int_{\mathcal{S}} \int_{\mathcal{R}^{2}} e^{-i \vec{x} \cdot \vec{k}} v(\theta, \vec{x} \cdot \vec{\theta}) d^{2} x \delta(\vec{k}-\sigma \vec{\theta}) d \theta \\
& =(2 \pi)^{-1} \int_{\mathcal{S}} \int_{\mathcal{R}^{2}} e^{-i \vec{x} \cdot \vec{k}} v(\theta, \vec{x} \cdot \vec{\theta}) d^{2} x \delta(\vec{k}-\sigma \vec{\theta}) d \vec{k} \sigma^{-1} \\
& =(2 \pi)^{-1} \int_{\mathcal{R}^{2}} e^{-i \sigma \vec{x} \cdot \vec{\theta}} v(\theta, \vec{x} \cdot \vec{\theta}) d^{2} x \sigma^{-1}
\end{aligned}
$$

We have also that $d(\vec{x} \cdot \vec{\theta})=d \vec{x} \cdot \vec{\theta}$ for constant $\vec{\theta}$. Thus:

$$
\begin{aligned}
F(\sigma \vec{\theta})[V(\vec{x})] & =(2 \pi)^{-1} \int_{\mathcal{R}^{2}} e^{-i \sigma \vec{x} \cdot \vec{\theta}} v(\theta, \vec{x} \cdot \vec{\theta}) d(\vec{x} \cdot \vec{\theta})(\sigma|\vec{\theta}|)^{-1} \\
& =(2 \pi)^{-1} \int_{\mathcal{R}^{2}} e^{-i \sigma z} v(\theta, z) d z(\sigma|\vec{\theta}|)^{-1} \quad(\text { where } z=\vec{x} \cdot \vec{\theta})
\end{aligned}
$$

The $z$ dependent terms now form a Fourier transform with respect to the second variable in $v$. Hence, we may write the above in the following form to elucidate the precise relation between $V$ and $v$ in Fourier terms:

$$
F(\sigma \vec{\theta})[V(\vec{x})]=(2 \pi)^{-1} F_{z}(\sigma)[v(\theta, z)](\sigma|\vec{\theta}|)^{-1}
$$

The effect of the back-projection operator on the Radon transform of $f$ may then be appreciated, via a consideration of equation 4 , by setting $v$ to be a Dirac delta function in 
$s$ (corresponding to an identity operation within the convolution). The $V$ corresponding to this $v$ may then be deduced by inserting the Fourier transform of the delta function (unity throughout $f$-space) into the above equation. Hence, we see that the effect of applying the back-projection operator to the Radon transformed $f$ function is the equivalent of convolving $f$ with the inverse Fourier-transformed remainder:

$$
f_{\text {recovered }}(x, y)=f_{\text {original }} * F^{-1}\left(s^{-1}\right)
$$

In terms of the tomographic analogy, we retrieve a "blurred" version of the original data. In fact, the object of tomography is exactly the reverse of this process: we seek to obtain a $v$ function such that it is $V$ that approaches the form of the delta function: that is, transforming the RHS of equation 4 into $f$ alone. In this instance, we may regard the $v$ function as a "filtering operator" that serves to remove morphology attributable to the sampling geometry rather than the original data, which is then hence applied to the Radon data at a stage prior to inversion via the back projection operator.

We shall in section 2.4 set out to show that the summation method of classifier combination (which is representative of many more generalized combination approaches under certain conditions, such as very limited class information within the individual classifiers) is, in effect, the equivalent of applying the back-projection operator immediately to the classifier PDFs (which in our analogy are to be considered Radon transforms), without any attempt to apply prior filtering (ie, setting $v$ to the delta function in equation 4). It is then via this observation that we hope to improve on the combination process, presenting an optimal, or near optimal solution to the inversion problem by finding an appropriate filter, $v$, albeit in the context of probability theory. 
Prior to setting out this correspondence we shall first extend the method to the $j$ dimensions required of our pattern vector, and illustrate how the mechanics of the Radon reconstruction might be applied within the current context.

\subsection{Generalization of the Radon Transform to Arbitrary Di- mensionality}

It would seem on intuitive grounds that there should exist a relatively straightforward generalization of the inverse Radon transform to an arbitrary number of dimensions, one that would, in theory, involve only a simple multi-dimensional extrapolation of the "deblurring" mechanism (here presumed to be a convolving filter) to generate a complete reconstruction of the original data. However, this would rely on having previously obtained a complete set of hyper-"facet" data, collectively defining the hyper-volume of pattern data via inverse Radon transformation (by way of three-dimensional illustration; we should have had to obtain three separate data sets consisting of perpendicular line integral bundles over a cubic pattern-space, the line-integrals being parallel to the three feature axes: that is, if we labeled the feature axes $a, b$ and $c$, then we could compute a 3d Radon reconstruction only if we have separately obtained all integrals perpendicular to the facets $a b, b c$ and $c d$ ).

This is of no immediate use for our methodology, however; the nature of the integral set out in equation 1 means that for all dimensionality in excess of two, we are no longer implicitly dealing with line integrals, but rather area integrals, volume integrals, and so on; the dimensionality attributable to each classifier, however, remains at the specified value of unity. If we are then to address this shortfall and reconstruct the complete pat- 
tern space via Radon methods, we shall require a series of intermediate stages in which the dimensionality of the pattern space is progressively increased prior to the final application of the $n$-dimensional inverse Radon transform alluded to above. In fact, these intermediate stages are themselves inverse multi-dimensional Radon transforms, providing an elegant continuity of mechanism across the dimensional range. Indeed, it further transpires that we might, rather than speculating on the nature of the dimensionallygeneralized inverse Radon transform, instead further break the problem into a series of standard two-dimensional Radon transforms.

Before we outline the methodology, we note that it may be objected at this stage, given that we have obtained the line integrals for every hyper-facet of the pattern space from data sets that were originally of one dimension, that the data contained within these hyper-facets must be of a highly correlated nature, and that the consequent pattern space reconstructed from them cannot conceivably then consist of independent data points. While this is certainly true, there is, however, a still more fundamental reason for which we must abandon the idea of independent points within the pattern space, and one that ultimately subsumes this point; namely, the mismatch between the angular and spatial sampling densities. Hence, even for data of two dimensions, the angular resolution of the Radon line integrals ( $\frac{\pi}{2}$ radian intervals) is such that the pattern space that we are trying to reconstruct almost invariably contains more independent information than the Radon transforms can possibly supply (respectively $n^{2}$ as opposed to $2 n$ independent variables, for line integrals consisting of $n$ parallel bundles).

This, it must be understood, does not in anyway represent a shortcoming of the method; the pattern space PDF that we are trying to reconstruct does not in any accessible sense have a prior existence in relation to which our method might be considered to be 
producing an approximation: the space is, in fact, constructed from discrete classifiers which have had their features chosen via individual preference, the implication being that feature dimensions not included have been so excluded because they fail to correctly represent the various class PDF morphologies. Therefore, the only non a priori sense in which an $n$-dimensional class PDF morphology could be said to exist is as a perfectly executed inverse radon transform of the features' class morphologies as represented by the individual classifiers.

We should add that, in the case of more than one feature belonging to a particular classifier (which we have excluded in the preceding discussion for simplicity), clearly, the class morphology will be well represented at the appropriate dimensionality without the need for any reconstructive method and, moreover, with truly independent data points: the reconstructive methods are only required for features contained in separate, and therefore mathematically independent classification schemes. Any such classifiers containing multiple features will then in fact enhance the reconstructive process, containing, as they do, entirely uncorrelated multidimensional information. In general, however, we shall have to consider that the data in our pattern space is inherently correlated in some sense: following the immediately succeeding outline of the proposed technique for multidimensional Radon inversion, we shall show that the pattern space may be considered via Fourier analogy as inherently "bandwidth limited".

We shall, then, in commencing our elucidation of the $n$-dimensional Radon transform, switch instead to a discretized version of equation 4, evaluating the integrals on the righthand side via the trapezoid rule, as is more appropriate to the computational nature of our reconstruction: this will also assist later in the quantification of the bandwidth limitation of the reconstructed data. Thus, equation 4 becomes: (after Natterer, 1996 
[8])

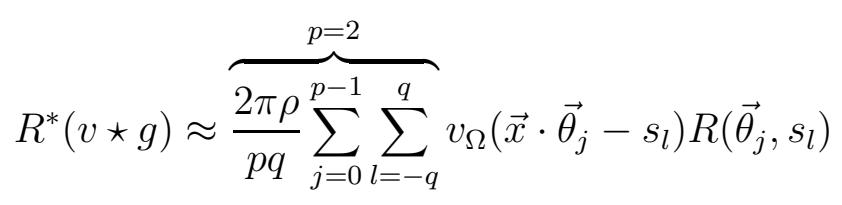

(the subscript $\Omega$ appended here to indicate some, as yet unspecified, bandwidth limitation).

We should also understand at this point that the principle of Radon transformation will not be modified by the fact that the data points of the reconstructed space now represent integrals over the remaining $n-2$ dimensions of the pattern space, rather than discrete points within a Euclidean space, the distinction being at this stage purely semantic.

We shall assume here that the function $v_{\Omega}$ is such that its adjoint $V$ has no effect on the real-space data, $f$, under convolution. Note that with the sampling function of the real data now explicitly included (that is, convolution with the "Dirac forest" or shah function), this condition is now no longer so restrictive as to imply that $V$ is solely a delta function. Indeed, any function with an origin value of unity and zeros at intervals corresponding to the peaks of the sampling function will have this property, perhaps the prime example being the (appropriately scaled) sinc function: any function that has a Fourier transform unity-valued up to the bandwidth limitation (corresponding to the reciprocal of the sampling rate) will, however, give rise to a function of the desired properties.

The first stage in the construction of the $n$-dimensional pattern space (for $n>3$ ) is the generation of every possible subset of two-dimensional pattern spaces from the total feature set of $n$ possibilities. Note that, as we have indicated, the reconstructed space will not strictly be an independent pattern space, every data point denoting an integral 
over the remaining $n-2$ dimensions. There will then be $\frac{n !}{(n-2) ! 2 !}={ }^{n} C_{2}$ such spaces, which we will henceforth label compositely in terms of their constituent feature sets, features being denoted in the following discussion by lower case Greek letters. Hence we require:

$$
\frac{\pi \rho_{\alpha \beta}}{q_{\alpha \beta}} \sum_{j_{\alpha \beta}=0}^{1} \sum_{l_{\alpha \beta}=-q_{\alpha \beta}}^{q_{\alpha \beta}} v_{\Omega}\left(\vec{x}_{\alpha \beta} \cdot \vec{\theta}_{\alpha \beta j}-s_{\alpha \beta l}\right) R\left(\vec{\theta}_{\alpha \beta j}, s_{\alpha \beta l}\right)
$$

for:

$$
\forall \alpha, \beta: \alpha, \beta \in \mathcal{I} ; \alpha \neq \beta ; 0<\{\alpha, \beta\}<n
$$

On the assumption that the prior geometry filtering process is executed perfectly, we will then have then obtained the optimal representation of the series of two-dimensional arrays of integrals over the remaining $n-2$ dimensions that have been excluded by each of the feature pair combinations. We must now construct every possible three dimensional pattern space from these two-dimensional data sets. If we had a generalized threedimensional inverse Radon transform (as well as access to a three-dimensional deblurring function) we might then construct each three-dimensional pattern space from the totality of its "facets" (that is, for features labeled $\alpha, \beta$ and $\gamma$, we would require the facets $\alpha \beta$, $\gamma \beta$ and $\gamma \alpha$ ), there being in general ${ }^{j} C_{j-1}=j$ such entities required for construction of a $j$ dimensional object (as we shall demonstrate later).

However, in the interests of maintaining continuity of mechanism over every dimensional iteration of the process, we shall instead address this as a series of two-dimensional inverse Radon transforms, the plane of operation of which being in each case that perpendicular to every set of line-integral bundles. In this way every point of the threedimensional space (not yet a pattern space until dimension $n$ is reached) is subject to an inverse Radon computation. Moreover, since there are three possible perpendicular 
planes through every given point of the space, we in fact have a surplus of two inverse Radon computations per data point. We shall not, at this stage, make any assumptions as to the precise interrelation of the separate point computations, but rather seek to unify them in a manner congruent with the overall Radon analysis: Since the essential nature of the Hilbert-space adjoint to the Radon transformation (ie, the unfiltered inverse Radon transform or back-projection operator) is, broadly speaking, one of addition followed by normalization, the appropriate method of combination for the three points would then seem to be the intuitive one of taking their collective mean (recall that geometry filtering process has already been applied in the derivation of each of the individual points).

This composite three dimensional inverse Radon transform would therefore appear in terms of the format of equation 9 as follows:

$$
\begin{gathered}
\frac{1}{3} \frac{\pi \rho}{q_{\alpha \beta}} \sum_{j_{\alpha \beta}=0}^{1} \sum_{l_{\alpha \beta}=-q_{\alpha \beta}}^{q_{\alpha \beta}} v_{\Omega}\left(\vec{x}_{\alpha \beta} \cdot \vec{\theta}_{\alpha \beta j}-s_{\alpha \beta l}\right) R\left(\vec{\theta}_{\alpha \beta j}, s_{\alpha \beta l}\right)+ \\
\frac{1}{3} \frac{\pi \rho^{\prime}}{q_{\gamma \beta}} \sum_{j_{\gamma \beta}^{\prime}=0}^{1} \sum_{l_{\gamma \beta}^{\prime}=-q_{\gamma \beta}}^{q_{\gamma \beta}} v_{\Omega}^{\prime}\left(\vec{x}_{\gamma \beta} \cdot \vec{\theta}_{\gamma \beta j^{\prime}}-s_{\gamma \beta l^{\prime}}\right) R\left(\vec{\theta}_{\gamma \beta j^{\prime}}, s_{\gamma \beta l^{\prime}}\right)+ \\
\frac{1}{3} \frac{\pi \rho^{\prime \prime}}{q_{\gamma \alpha}} \sum_{j_{\gamma \alpha}^{\prime \prime}=0}^{1} \sum_{l_{\gamma \alpha}^{\prime \prime}=-q_{\gamma \alpha}}^{q_{\gamma \alpha}} v_{\Omega}^{\prime \prime}\left(\vec{x}_{\gamma \alpha} \cdot \vec{\theta}_{\gamma \alpha j^{\prime \prime}}-s_{\gamma \alpha l^{\prime \prime}}\right) R\left(\vec{\theta}_{\gamma \alpha j^{\prime \prime}}, s_{\gamma \alpha l^{\prime \prime}}\right) \\
\forall \alpha, \beta: \alpha, \beta \in \mathcal{I} ; \alpha \neq \beta ; 0<\{\alpha, \beta\}<n
\end{gathered}
$$

However, we should recall that the angular ordinate $j$ may take only two values, dividing the plane into two perpendicular axes. If we can then permit a further simplifying assumption, to serve merely as a vehicle for elucidating the various redundancies and symmetries inherent in the above mathematical structure, namely, that there are equal numbers of data points in each of the Radon transforms (ie that the various $q$ are all equal in the above), then we see that the planes become pairwise-degenerate. That is, 
we find the relations:

$$
\theta_{\gamma \alpha 1} \equiv \theta_{\alpha \beta 1}, \theta_{\gamma \beta 1} \equiv \theta_{\alpha \beta 0}, \theta_{\gamma \beta 0} \equiv \theta_{\gamma \alpha 0}
$$

and

$$
s_{\gamma \alpha 1} \equiv s_{\alpha \beta 1}, s_{\gamma \beta 1} \equiv s_{\alpha \beta 0}, s_{\gamma \beta 0} \equiv s_{\gamma \alpha 0}
$$

If we make the related assumption that the deblurring functions are symmetric under rotation (the rotational symmetry of the geometry should guarantee this for equivalent linear axes) then we should, in conjunction with the previous assertion, have a further degeneracy in the form of an equality of action among the various deblurring functions (that is; $\left.v_{\Omega}=v_{\Omega}^{\prime}=v_{\Omega}^{\prime \prime}\right)$.

We may yet make a further simplification to equation 9 in that, having labeled the planebound vectors $\vec{x}$ and $\vec{\theta}$ by the double Greek subscripts that denote their constitutive features, a redundancy in the dot product $\vec{x} \cdot \vec{\theta}$ makes itself apparent, the plane-specific nature of the one quantity invariably implying the plane-specific nature of their product. We are then free to generalize either one of the vectors to a unit higher dimensionality without the loss of equations 10's veracity. We shall opt, then, to entirely omit the subscripts of $\vec{x}$, allowing it instead to represent the same arbitrary three-dimensional vector within each of the three summations of equation 9. That is, the original subscripted vectors will each become projections of a common, three-dimensional vector onto the host planes that originally contained them.

Along with the further trivial normalizing assumption that $q_{\alpha \beta}=q_{\gamma \beta}=q_{\gamma \alpha} \equiv q$, we have now the capacity to simplify equation 9 to approximately half of its complexity, 
giving us the equivalent form;

$$
\begin{gathered}
\frac{\pi}{3 q}\left(\rho+\rho^{\prime \prime}\right) \sum_{l_{\alpha \beta}=-q}^{q} v_{\Omega}\left(\vec{x} \cdot \vec{\theta}_{\alpha \beta 0}-s_{\alpha \beta l}\right) R\left(\vec{\theta}_{\alpha \beta 0}, s_{\alpha \beta l}\right)+ \\
\frac{\pi}{3 q}\left(\rho+\rho^{\prime}\right) \sum_{l_{\alpha \beta}^{\prime}=-q}^{q} v_{\Omega}\left(\vec{x} \cdot \vec{\theta}_{\alpha \beta 1}-s_{\alpha \beta l^{\prime}}\right) R\left(\vec{\theta}_{\alpha \beta 1}, s_{\alpha \beta l^{\prime}}\right)+ \\
\frac{\pi}{3 q}\left(\rho^{\prime}+\rho^{\prime \prime}\right) \sum_{l_{\gamma \beta}^{\prime \prime}=-q}^{q} v_{\Omega}\left(\vec{x} \cdot \vec{\theta}_{\gamma \beta 1}-s_{\gamma \beta l^{\prime \prime}}\right) R\left(\vec{\theta}_{\gamma \beta 1}, s_{\gamma \beta l^{\prime \prime}}\right) \\
\forall \alpha, \beta: \alpha, \beta \in \mathcal{I} ; \alpha \neq \beta ; 0<\alpha, \beta<n,
\end{gathered}
$$

which will permit us later to make very general statements about the higher dimensional equivalents of equation 9 .

Even if we do not make any of the above assumptions, the only way in which the actual situation may differ from the imposed one is in terms of the extent of, and the number of, the data points within each particular feature. It is fairly evident from the preceding discussion that this may be accommodated very simply within equation 14 via an appropriate rebinning and normalization of each axis, without compromising any of the symmetry related simplifications that we have discussed.

We have then, in consequence of this elimination of redundancy, the basis for constructing a method of $n$-dimensional inverse Radon transformation from $(n-1)$-dimensional pattern data, the only difference in the more general mechanism being the number of constitutive $(n-1)$-dimensional entities required to fully describe the reconstituted $n$ dimensional object. The precise number will simply be the number of non-permuted subsets of $n-1$ objects that we can draw from a total of $n$ distinct objects, this being ${ }^{n} C_{n-1}$, corresponding to the number of $(n-1)$-dimensional hyper-facets required to uniquely specify the points of an $n$-dimensional hyper-cube, and is in fact the same as the number of feature dimensions in the initial data $\left(\right.$ since $\left.{ }^{n} C_{n-1}=\frac{n !}{(n-1) ! 1 !}=n\right)$. Thus 
the number of summations in the $n$-dimensional generalization of equation 14 will be $n$, and the number of Greek subscripts that will need to be associated with any given $\theta$ or $s$ is $n-1$.

Therefore, rather than writing out a very general composite equation embodying this principle, we have instead a series of progressive stages of association of the $n$ separate feature dimensions, the dimensionality of the entities constructed at each step increasing by a factor of one, until the cumulating $n$-dimensional object is finally created. Needless to say, notwithstanding our simplification of the procedure by insisting on the allocation only one feature per classifier (condition 2), the method can accommodate (and is in fact very much improved by) having to subsume within it classifiers with more than one feature. In this case there is, of course, no need for reconstruction of the corresponding pattern space via Radon methods; the space is already fully and accurately defined. In such a regime we may then simply skip the Radon reconstruction procedure corresponding to those particular dimensions in the above construction, without the loss of methodical consistency.

Aside from its complexity, this latter point is then the predominating reason for not having explicitly compiled a general equation for the execution of the $n$-dimensional inverse Radon transformation from its one-dimensional constituents favoring, rather, its retention as a recursive computational procedure. This point shall be fully realized in section 3.4.3, where we set out a fully general economized tomographic classifier fusion procedure centered around the principle of graphical intercorrelation. 


\subsection{Sampling Concerns}

We now return to the question of what conditions, if any, the very low number of angular Radon samples inherent in our class probability density functions will impose upon the reconstructed pattern space, or even upon the very utility of the method itself, given that we shall have to consider our reconstructed data exceptionally band-width limited in some sense, within the terms of the Fourier analogy implied by equation 6 .

In the interests of clarity, and given our recursive approach, we shall consider this bandwidth limitation only in terms of the individual two-dimensional inverse Radon transforms from which the multi-dimensional inverse transform is progressively constructed, the $n$-dimensional corollary of these findings being at least qualitatively self-evident, and the predictions for the utility of the technique remaining valid by extension.

There are then two distinct aspects to the sampling issue as it relates to Radon transformation, namely, the linear and the rotational integrations within the discretized form of the inverse Radon transform (equation 8). The first of these we can address in terms of the Nyquist criterion for sufficient sampling within the Fourier domain; following Natterer (1986) [8] we shall consider that the reconstructed pattern space is bandwidth limited (in the Fourier sense) to frequencies within a value $\Omega$. The Nyquist criterion states that this space may then be fully determined by linear sampling with a step-size of $\leq \pi / \Omega$. In the nomenclature of equation 8 , this step-size may be derived from the width of the reconstructed space, $\rho$, and the total number of parallel Radon transforms, $q$, via the ratio $\rho / q$. The fact that the Radon transform and the pattern space have identical bandwidth limitations, as is implicitly considered to be the case in the above argument, may be verified by an inspection of equation 6 , which linearly relates the 
Fourier transforms of the two quantities. The overall imposition upon the bandwidth arising from these arguments may then be derived from the two step-size related terms to be:

$$
q \geq \frac{1}{\pi} \rho \Omega
$$

However, we have also to consider what possible bandwidth limitations are imposed by the rotational sampling rate, which, given that Radon samples are obtained for only two angles per plane of reconstruction (the feature axes), would then appear, on intuitive grounds, to be the dominating factor of the two.

This calculation is less straightforward, and we adopt Natterer's (1986) [8] argument in terms of Bessel functions by way of approximation. Using Debye's representation of the asymptotic form of Hankel functions of the the first kind as a method of relating angular integration to wavelength (or its nearest equivalent in Bessel terms), it can be shown that the bandwidth of the Radon transform in terms of $\theta$ is essentially $\Omega \rho$. The step-size relevant to angular version of the Nyquist criterion is then simply $\pi / p$, the criterion itself consequently imposing the restriction: $\frac{\pi}{p} \leq \frac{\pi}{\rho \Omega}$, or:

$$
p \geq \rho \Omega
$$

Now $p$, as we have stated, is equal to two. The bandwidth criterion owing to the angular sampling rate is then: $\Omega \leq \frac{2}{\rho}$. From equation 15, however, the corresponding bandwidth criterion deriving from the linear sampling rate was simply: $\Omega \leq \frac{\pi q}{\rho}$. Now, the number of points in a typical classifier-derived PDF will generally be in excess of the cardinality of the test data set from which it derived; being typically of the order of 1000 . This, and the corresponding bandwidth limitation, will clearly be so far in excess of the 
angular sampling limitations that we are justified in entirely disregarding the number

of sample points as being of any consequence at all to the recovered pattern space morphology, being thus dominated almost completely by correlations imposed by the angular sampling rate. Hence, this is a pattern space of inherently few degrees of freedom in relation to the multi-dimensional feature spaces that exist within single classifiers, and thus no method of classifier combination, no matter how perfect (corresponding to having obtained a perfect "deblurring" filter, $v_{\Omega}$ ), can possibly reconstruct an $n$ dimensional pattern space consisting of entirely independent points.

\subsection{Formal Explication of the Parallel between Radon Trans- form Theory and Classifier Combination}

Having obtained a mathematical form (or rather, a method) for $n$-dimensional inverse Radon transformation, we are now in a position to make the correspondence of the outlined method to classifier combination theory more formally explicit. That is, we shall seek to encompass the various extant combinatorial decision theories within the tomographic framework that we have developed over the preceding sections, and show that they represent, within certain probabilistic bounds, an imperfect approximation to the unfiltered inverse Radon transformation.

We will firstly, however, demonstrate how we might explicitly substitute probabilistic terms into equation 14 , and therefore, by extension, the complete $n$-dimensional inverse Radon transformation. We have initially then to establish exactly what is meant in geometrical terms by the Radon forms upon which equation 14 is constructed. It is helpful in this endeavor to, at least initially, eliminate the complication of the pre- 
filtering convolution represented by $v$. We do this by setting $v$ to a discretized form of the Dirac $\delta$ function throughout the summation, $\sum_{l_{x y}=q}^{q}$, that is:

$$
\begin{aligned}
& v_{\Omega}\left(s_{x y l}\right)=\frac{1}{\sum_{l_{x y}=q}^{q} s_{x y l}}=\frac{1}{2 q} \text { when } s_{x y l}=0 \\
& v_{\Omega}\left(s_{x y l}\right)=0 \text { otherwise }
\end{aligned}
$$

Hence, the various summations only produce non-zero terms when:

$$
\begin{aligned}
\vec{x} \cdot \vec{\theta}_{\alpha \beta 0}-s_{\alpha \beta l} & =0 \\
& \text { or } \\
\vec{x} \cdot \vec{\theta}_{\alpha \beta 0} & =s_{\alpha \beta l}
\end{aligned}
$$

Thus, without filtering, equation 14 commutes to the form:

$$
\begin{gathered}
\frac{\pi}{3 q}\left(\rho+\rho^{\prime \prime}\right) R\left(\vec{\theta}_{\alpha \beta 0}, \vec{x} \cdot \vec{\theta}_{\alpha \beta 0}\right) \\
\frac{\pi}{3 q}\left(\rho+\rho^{\prime}\right) R\left(\vec{\theta}_{\alpha \beta 1}, \vec{x} \cdot \vec{\theta}_{\alpha \beta 1}\right) \\
\frac{\pi}{3 q}\left(\rho^{\prime}+\rho^{\prime \prime}\right) R\left(\vec{\theta}_{\gamma \beta 0}, \vec{x} \cdot \vec{\theta}_{\gamma \beta 0}\right) \\
\forall \alpha, \beta: \alpha, \beta \in \mathcal{I} ; \alpha \neq \beta ; 0<\alpha, \beta<n
\end{gathered}
$$

For convenience we shall normalize the axial extent parameters; $\rho=\rho^{\prime}=\rho^{\prime \prime}$ throughout the following discussion, such that we may introduce a constant multiplying factor, $A$, into each summation component.

Now, because we a free to set the coordinate system as we choose, and, in having set $j$ to 
2 in equation 14, consequently obtaining a perpendicularity between the Radon integral vectors, we shall find it convenient to express our geometry in terms of an orthogonal coordinate system, with axial direction vectors set parallel to the perpendicular Radon integrals. Thus, we may legitimately make the equations:

$$
\alpha=x_{1}, \beta=x_{2}, \gamma=x_{3}
$$

Also, in having imposed this parallelism between the Radon integrals and coordinate axes, we find that the subscript $x y l$ comes to exhibit a redundancy of two variables, such that we may state the further consequent equivalences:

$$
\alpha \beta 0=\alpha, \alpha \beta 1=\beta, \gamma \beta 0=\gamma
$$

Thus equation 14 now adopts the form:

$$
A\left[R\left(\vec{\theta}_{x_{1}}, x_{1}\right)+R\left(\vec{\theta}_{x_{2}}, x_{2}\right)+R\left(\vec{\theta}_{x_{3}}, x_{3}\right)\right]
$$

However, recall from equation 2 that:

$$
R(\theta, s)\left[f\left(x_{1}^{\prime}, x_{2}^{\prime}\right)\right]=\int_{-\infty}^{+\infty} \int_{-\infty}^{+\infty} f\left(x_{1}^{\prime}, x_{2}^{\prime}\right) \delta\left(s-x_{1}^{\prime} \cos \theta-x_{2}^{\prime} \sin \theta\right) d x_{1}^{\prime} d x_{2}^{\prime}(=g(s, \theta))
$$

Now, we also have that;

$$
\begin{aligned}
& \cos \vec{\theta}_{x_{2}}=\sin \vec{\theta}_{x_{1}}=0 \\
& \text { and } \\
& \cos \vec{\theta}_{x_{1}}=\sin \vec{\theta}_{x_{2}}=1 .
\end{aligned}
$$


( $\theta$ being measured in relation to the $x_{1}$ axis)

Thus, for example, picking an ordinate at random:

$$
\begin{aligned}
R\left(\vec{\theta}_{x_{1}}, x_{1}\right) & =\int_{-\infty}^{+\infty} \int_{-\infty}^{+\infty} f\left(x_{1}, x_{2}\right) \delta\left(x_{1}-x_{1}^{\prime}\right) d x_{1}^{\prime} d x_{2}^{\prime} \\
& =\int_{-\infty}^{+\infty} f\left(x_{1}, x_{2}^{\prime}\right) d x_{2}^{\prime} \\
& =\int_{-\infty}^{+\infty} f\left(x_{1}, x_{2}\right) d x_{2}
\end{aligned}
$$

and similarly for $x_{2}, x_{3}$

Now, a rational extension of the nomenclature of equation 1 would allow us to write:

$$
p\left(x_{1}, x_{2} \mid \omega_{i}\right) d x_{k}=\int_{-\infty}^{+\infty} \underbrace{\ldots}_{n-2} \int_{-\infty}^{+\infty} p\left(X \mid \omega_{i}\right) d x_{3} \ldots d x_{R} \cdot d x_{1} d x_{2}
$$

(and similarly for the remaining pairs of basis vector combinations)

We, of course, still have that:

$$
p\left(x_{1} \mid \omega_{i}\right) d x_{k}=\int_{-\infty}^{+\infty} \underbrace{\ldots}_{n-1} \int_{-\infty}^{+\infty} p\left(X \mid \omega_{i}\right) d x_{2} \ldots d x_{R} \cdot d x_{1}=\int_{-\infty}^{+\infty} p\left(x_{1}, x_{2} \mid \omega_{i}\right) d x_{2}
$$

Thus, by setting the equivalence $f\left(x_{1}, x_{2}\right) \equiv p\left(x_{1}, x_{2} \mid \omega_{i}\right)$, we find by direct substitution into equation 24 that we can state that:

$$
R\left(\vec{\theta}_{x_{1}}, x_{1}\right)=\int_{-\infty}^{+\infty} f\left(x_{1}, x_{2}\right) d x_{2}=p\left(x_{1} \mid \omega_{i}\right)
$$

and similarly for the remaining numeric subscripts.

Hence, in consequence, we may simply restate the unfiltered two-to-three dimensional 
inverse Radon transformation in the more transparent form:

$$
A\left[p\left(x_{1} \mid \omega_{i}\right)+p\left(x_{2} \mid \omega_{i}\right)+p\left(x_{2} \mid \omega_{i}\right)\right]
$$

Moreover, we can go further and extend this approach to the recursive methodology of the $n$-dimensional inverse Radon transformation, in which case we find in the most general terms, that the unfiltered $n$-dimensional inverse Radon transformation will have the form (declining explicit calculation of the various normalizing constants corresponding to $A$ in the above, this being a relatively complex undertaking, and not in any case required in the context of the decision making schemes within which the method will ultimately be applied [see later]):

$$
A^{\prime}\left[\sum_{\text {all k }} p\left(x_{k} \mid \omega_{i}\right)\right]
$$

which clearly comes to resemble the Sum Rule decision making scheme (a correspondence we shall make formal later).

The substitution of probabilistic terms into the generalized inverse Radon transformation having thus been rendered explicit, it is now an elementary matter to substitute the previously omitted filtering function $v_{\Omega}$ back into equation 29 (the various subscript redundancies induced by an appropriate selection of the coordinate system above applying equally to the variable $s$ in equation 14), most particularly since the set of filtering convolutions will remain additive in relation to their correspondent $p\left(x_{k} \mid \omega_{i}\right)$ functions throughout the recursive increment in dimensionality, and will therefore readily generalize to a composite $n$-dimensional filtering function. (We omit a discussion of its specific form since this is entirely dependent on the choice of $v_{\Omega}$ ). 
Having transcribed the inverse Radon transform into purely probabilistic terms and eliminated any residual geometric aspects of the problem, we may now turn to an investigation of how the $n$-dimensional reconstruction relates to the decision making process implicit within every regime of classifier combination.

As a preliminary to this endeavor, we must firstly ensure that there exist comparable pattern vectors for each class PDF (such not necessarily being the case for feature sets constructed on a class-by-class basis, as within our approach). That is, we shall need to ensure that:

$$
p\left(x_{R_{i}(1)}, \ldots, x_{R_{i}\left(j_{k, i}\right)} \mid \omega_{k}\right)=p\left(x_{l_{k}}, \ldots, x_{u_{k}} \mid \omega_{k}\right) \forall i, k
$$

where $u_{k}$ and $l_{k}$ are, respectively, the highest and lowest feature indices of the various feature sets involved in the combination, and $j_{k, i}$ is the cardinality of the feature set corresponding to the $k$ th class and $i$ th classifier: $R_{i}\left(n_{k, i}\right)$ is then the $n$th highest feature index in the feature set presented to the $i$ th classifier for computation of class PDF number $k$.

This may be straightforwardly accomplished by the inclusion of null vector components, such that:

$$
p\left(x_{t \notin R_{i}\left(\leq j_{k, i}\right)} \mid \omega_{k}\right)=\frac{1}{\int d x_{1} d x_{2} \ldots d x_{t-1} d x_{t+1} \ldots d x_{N}} d x_{t} \forall t, i, k
$$

implicitly setting $l_{k}$ to 1 and $u_{k}$ to $N$, thereby allowing a universal approach for each class index, $k$

Now, we have via the Bayes decision rule (ie that:

$$
\operatorname{assign} \quad \vec{X} \rightarrow \omega_{j} \quad \text { if }
$$




$$
p\left(\omega_{j} \mid x_{1}, \ldots x_{N}\right) \quad=\quad \max _{k} p\left(\omega_{j} \mid x_{1}, \ldots x_{N}\right),
$$

given;

$$
p\left(\omega_{k} \mid x_{1}, \ldots x_{N}\right)=\frac{p\left(x_{1}, \ldots, x_{N} \mid \omega_{k}\right) p\left(\omega_{k}\right)}{p\left(x_{1}, \ldots, x_{N}\right)}
$$

), that our decision rule for unfiltered $N$-dimensional inverse Radon PDF reconstruction is:

$$
\begin{aligned}
\operatorname{assign} \quad \vec{X} & \rightarrow \omega_{j} \quad \text { if } \\
p\left(\omega_{j} \mid x_{1}, \ldots x_{N}\right) & =\max _{k}\left[\frac{\sum_{i=1}^{N} p\left(x_{i} \mid \omega_{k}\right) p\left(\omega_{k}\right)}{p\left(x_{1}, \ldots, x_{N}\right)}\right]
\end{aligned}
$$

(from equation 29)

The more familiar decision rules, however, may be derived solely via probabilistic constraints on the Bayes decision rule. For instance, suppose that we impose the condition that $x_{1}, \ldots, x_{N}$ are independent random variables (such that:

$$
p\left(x_{1}, \ldots, x_{N} \mid \omega_{k}\right)=\prod_{i=1}^{N} p\left(x_{i} \mid \omega_{k}\right)
$$

), then we obtain the decision rule:

$$
\begin{aligned}
\operatorname{assign} \quad \vec{X} & \rightarrow \omega_{j} \quad \text { if } \\
p\left(\omega_{j} \mid x_{1}, \ldots x_{N}\right) & =\max _{k}\left[\frac{\prod_{i=1}^{R} p\left(x_{i} \mid \omega_{k}\right) p\left(\omega_{k}\right)}{p\left(x_{1}, \ldots, x_{R}\right)}\right]
\end{aligned}
$$

That is, we obtain the classical "Product Rule". 
If we impose the further constraint that:

$$
p\left(\omega_{k} \mid x_{i}\right)=p\left(\omega_{k}\right)\left[1+\delta f\left(\omega_{k}, x_{i}\right)\right]
$$

with $\delta f\left(\omega_{k}, x_{i}\right)$ an infinitesimal function (in effect, imposing a high degree of "overlap" among the total set of class PDFs, or, equivalently, a ubiquitous class membership ambiguity), and apply this directly to the Bayes theorem for single vectors:

$$
p\left(\omega_{k} \mid x_{i}\right)=\frac{p\left(x_{i} \mid \omega_{k}\right) p\left(\omega_{k}\right)}{p\left(x_{i}\right)}
$$

then we obtain:

$$
p\left(\omega_{k} \mid x_{i}\right)=\frac{p\left(x_{i} \mid \omega_{k}\right) p\left(\omega_{k}\right)}{p\left(x_{i}\right)}=\frac{p\left(\omega_{k}\right)\left[1+\delta f\left(\omega_{k}, x_{i}\right)\right]}{p\left(x_{i}\right)}
$$

Or, more succinctly:

$$
\left[1+\delta f\left(\omega_{k}, x_{i}\right)\right]=p\left(x_{i} \mid \omega_{k}\right)
$$

Substituting back into equation 36, the Product Rule decision scheme, obtained, we recall, via the imposition of statistical independence among a given class's pattern vector ordinates, we then have the resultant decision rule:

$$
\begin{aligned}
\operatorname{assign} \quad \vec{X} & \rightarrow \omega_{j} \quad \text { if } \\
p\left(\omega_{j} \mid x_{1}, \ldots x_{N}\right) & =\max _{k}\left[\frac{\prod_{i=1}^{R}\left[1+\delta f\left(\omega_{k}, x_{i}\right)\right] p\left(\omega_{k}\right)}{p\left(x_{1}, \ldots, x_{R}\right)}\right]
\end{aligned}
$$

Expanding the product and collecting infinitesimals of higher order (via the function 
$O(2))$, we obtain:

$$
\begin{aligned}
\operatorname{assign} \quad \vec{X} & \rightarrow \omega_{j} \text { if } \\
p\left(\omega_{j} \mid x_{1}, \ldots x_{N}\right) & =\max _{k}\left[\frac{\left[1+\sum_{i=1}^{R} \delta f\left(\omega_{k}, x_{i}\right)+O(2)\right] p\left(\omega_{k}\right)}{p\left(x_{1}, \ldots, x_{R}\right)}\right]
\end{aligned}
$$

Eliminating $O(2)$, and re-substituting equation 40, we find:

$$
\begin{aligned}
\operatorname{assign} \quad \vec{X} & \rightarrow \omega_{j} \text { if } \\
p\left(\omega_{j} \mid x_{1}, \ldots x_{N}\right) & =\max _{k}\left[\frac{\left[1+\sum_{i=1}^{R}\left\{p\left(x_{i} \mid \omega_{k}\right)-1\right\}\right] p\left(\omega_{k}\right)}{p\left(x_{1}, \ldots, x_{R}\right)}\right]
\end{aligned}
$$

Or:

$$
\begin{aligned}
\operatorname{assign} \quad \vec{X} & \rightarrow \omega_{j} \quad \text { if } \\
p\left(\omega_{j} \mid x_{1}, \ldots x_{N}\right) & =\max _{k}\left[\frac{(1-R) p\left(\omega_{k}\right)}{p\left(x_{1}, \ldots, x_{R}\right)}+\frac{\sum_{i=1}^{R} p\left(\omega_{k}\right)}{p\left(x_{1}, \ldots, x_{R}\right)}\right],
\end{aligned}
$$

which is the equivalent of the classical "Sum Rule":

$$
\begin{aligned}
\operatorname{assign} \quad \vec{X} & \rightarrow \omega_{j} \quad \text { if } \\
p\left(\omega_{j} \mid x_{1}, \ldots x_{N}\right) & =\quad \max _{k}\left[\frac{\sum_{i=1}^{R} p\left(\omega_{k}\right)}{p\left(x_{1}, \ldots, x_{R}\right)}\right]
\end{aligned}
$$

when the unconditional class probabilities $p\left(\omega_{k}\right)$ are close to equality.

This, however, is identical to our original decision rule for the unfiltered inverse Radon transformation. Hence, we may state that the unfiltered inverse Radon PDF reconstruction is, within a Bayesian decision-making context, the equivalent of the Sum Rule decision making scheme under the specified probabilistic constraints (and the minor ad- 
ditional imposition of the unconditional class probabilities having approached equality), and will thus produce near-optimal results only when the two conditions are satisfied (ie that the pattern vector components are statistically independent, and that there exists a high class membership ambiguity owing to similar PDF morphologies). The unfiltered inverse Radon decision making scheme then recreates the Product Rule under the less constrictive (and therefore more common) condition of a high class membership ambiguity alone, a condition, however, which must still presuppose very major constraints on the $N$-dimensional PDF morphology if the equality is to hold.

Very many other classical combination rules are derived from combinations of these preconditions (see [2]) and thus come to resemble, to some degree, the unfiltered inverse transform. Without exception, however, they will all impose very considerable constraints on the implied $N$-dimensional PDF reconstruction. When viewed in this morphological regard, it is clear that the lack of universal application of classical methods of combination, however effective they may be within their typical domains of application, is (by an inversion of the above process) attributable to these implicit constrictions on the reconstructive process, to which these methods have been shown to offer an approximation. The only way in which we can free ourselves of these restrictions (on the assumption that we have obtained error-free PDFs [see later]) is then to apply the filtered inverse Radon transform in its entirety, since this inherently neither assumes nor imposes any morphological (and therefore probabilistic) constraints on the final $N$-dimensional PDF, other than those already implicit in the original PDF data.

On model-theoretical grounds this would therefore represent an optimal solution to the implied problem of $N$-dimensional PDF reconstruction, having shown, by an inversion of the arguments above, that at least one aspect of every method of classifier combination 
is in some (not necessarily immediately obvious) way, the implicit recovery of an $N$ dimensional PDF.

We have now to consider whether the above argument is modified by the fact that the various classifier PDFs, in consequence of having been derived from a finite set of stochastically distributed pattern data points, will invariably, to some extent, deviate from the "true" (if only hypothetically existent) probability density functions:

\subsection{Issue of Estimation Error}

We have observed in the preceding section that the unfiltered inverse transform equates to the Sum Rule decision scheme (with some additional probabilistic constraints) and would thus appear, on purely reconstructive grounds, to be a rather poor method of combining classifiers. However, we find that this is not in general the case, the Sum Rule often, in fact, achieving a better classification performance than the Product Rule, despite inherently making less impositions on the form of the $N$-dimensional pattern PDF that has been implicitly reconstructed. The reason for this is that the Sum Rule exhibits a pronounced robustness to estimation errors, which we demonstrate in the following way (paralleling the discussion in Kittler et al 1998 [2], albeit in terms of the prior probabilities):

Denoting by $\hat{P}$ the hypothetical PDF from which the pattern data originally derived, we have that the PDF, $P$, constructed by the classifier under consideration is related to the accented quantity via the error value $e_{i j}$ as follows:

$$
\hat{P}\left(x_{i j} \mid \omega_{j}\right)=P\left(x_{i j} \mid \omega_{j}\right)+e_{i j}
$$


Now, by inspection of the decision rule formula, equation 45, we can extract the essential summation component of the Sum Rule to be the formula:

$$
\begin{aligned}
\sum_{i}^{n} \hat{P}\left(x_{i j} \mid \omega_{j}\right) & =\sum_{i}^{n}\left[P\left(x_{i j} \mid \omega_{j}\right)+e_{i j}\right] \\
& =\left[\sum_{i}^{n} P\left(x_{i j} \mid \omega_{j}\right)\right]\left[1+\frac{\sum_{i}^{n} e_{i j}}{\sum_{i}^{n} P\left(x_{i j} \mid \omega_{j}\right)}\right]
\end{aligned}
$$

The latter term in square brackets we denote the error factor.

The equivalent component to the summation above in Product Rule terms would then be the following:

$$
\begin{aligned}
\prod_{i}^{n} \hat{P}\left(x_{i j} \mid \omega_{j}\right)= & \prod_{i}^{n}\left(P\left(x_{i j} \mid \omega_{j}\right)+e_{i j}\right) \\
\approx & {\left[\prod_{i}^{n} P\left(x_{i j} \mid \omega_{j}\right)\right]\left[1+\sum_{i}^{n} \frac{e_{i j}}{P\left(x_{i j} \mid \omega_{j}\right)}\right] } \\
& \text { (assuming higher order } e_{i j} \text { terms to be negligible) }
\end{aligned}
$$

The latter square-bracketed term being then the error factor associated with the Product Rule.

We see immediately that, within the error factor for the Product Rule, each $e_{i j}$ is amplified by the term;

$$
\frac{1}{P\left(x_{i j} \mid \omega_{j}\right)}>>\frac{1}{P\left(\vec{X} \mid \omega_{j}\right)}
$$

as opposed to the Sum Rule, for which the corresponding amplification term is;

$$
\frac{1}{\sum_{i}^{n} P\left(x_{i j} \mid \omega_{j}\right)}=\frac{1}{P\left(\vec{X} \mid \omega_{j}\right)}
$$

Thus, the Sum Rule exhibits a very much greater degree of stability in relation to 
estimation errors. It may be shown (see [2]) that the Sum and Product Rules represent opposite poles, in terms of their sensitivity to errors, of a very large range of classifier combination techniques, most of the remaining combinatorial strategies being implicitly derived from either or both sets of the probabilistic assumptions that underly these two polar combination methods, and which hence fall somewhere between them in terms of their robustness to estimation error.

We would expect, then, given that unfiltered tomographic PDF reconstruction is essentially identical in operation to the Sum Decision Rule for classifier combination, that the process will exhibit a similar robustness to estimation error, and therefore, if it is indeed the case that the Sum Rule represents an optimal solution to the problem, a near optimal combinatorial method, both in this sense, as well as in the former sense of being an ideal information-theoretic $N$-dimensional PDF recovery procedure. In addition, because we have stated at the outset that poorly performing feature/classifier combinations are explicitly rejected at the feature selection stage (since they represent inherently poor models of the PDF), this argument becomes even more forceful.

We need, however, to consider the consequence of filtering on this argument if we are to make the case without reservation. In general this will not be simple, and will depend to a very great extent on the nature of the classifier. This is because filtering acts, in essence, as an edge enhancing, gradient-negating convolution (similar in effect to the familiar "Mexican hat" filter), and will thus act differently on differing classification procedures. For instance, errors caused by exceptional outliers within a "nearest-neighbor" classification framework would tend to be exacerbated by filtration, whereas a continuous curve-fitting classifier presenting too smooth a PDF might, in consequence, have its estimation error reduced by the act of filtration. We cannot, therefore, consider that 
there is any single systematic effect of the deblurring process on the final estimation error of the reconstructed $N$-dimensional PDF. Intuitively, however, we may state the error will remain of a similar order to that of the Sum Rule decision scheme (on the assumption of an overall cancellation of the negative and positive aspects of filtration): a more formal investigation of this point will constitute a later section of the article.

We should further add, at this point, that an exact morphological reconstruction of the various class PDFs within an $N$-dimensional pattern space will have less of a consequence for the overall performance of the decision scheme if the classes are well separated (which is why binary class delimiters such as neural networks, with PDFs that in consequence exhibit only two values of the probability density, namely zero and a constant, may yet still exhibit a good classification ability despite being unrepresentative of the "true" PDF). Thus conventional methods of combination might, within such a scenario, approach the theoretical performance levels of the tomographic method. We should reiterate, however, that the latter will always represent the optimal (in the sense of least biased) decision making scheme, albeit at some additional computational cost. Whether, in this instance, the full $N$-dimensional filtered Radon inversion would be justified for such a small increment in performance would be a matter of appropriate judgment. When, however, there exists a high degree of class membership ambiguity, the performance enhancement of the proposed technique should, we expect, be very marked.

In consequence of this absence of a general solution to the issue of estimation error, we shall in section 5, attempt to give practical and model-based measures of the resilience of the method to this source of error, finding, in doing so that our intuitive arguments of this section are, to a large extent, empirically justified. 


\subsection{Strategy for the Combination of Overlapping Feature Sets}

We have thus far considered tomographic reconstruction theory only in terms of distinct feature sets: the contrary situation must now be addressed if we are to arrive at a universally optimized solution to the problem of classifier combination. Before embarking on this investigation we should, however, reiterate just how exceptional it is to find overlapping feature sets among the classifiers within a combination when feature selection is explicitly carried out within a combinatorial context (see [11]).

The specific question that we are seeking to address is therefore what strategy to adopt when presented with overlapping feature sets on attempting our tomographic reconstruction of the complete $N$-dimensional pattern-space probability density function. This is clearly is not a problem for classical methods of combination, which consider combination in probabilistic, not morphological, terms, and which do not thus consider the implicit ambiguity in PDF representations as presenting any particular difficulty. Indeed, classical techniques such as majority voting may actively assist us in our tomographic endeavor by explicitly eliminating superfluous PDF characterizations, and, as such, are not in any way mutually exclusive to our methodology. In general, though, it will not be obvious whether the current and classical methodologies are of simultaneous applicability.

There is, however, another perspective from which we may view the action of classical combination methods in regard to overlapping feature sets (as opposed to implicit unfiltered tomographic reconstruction, which would only apply to distinct feature sets), and that is as methods for refining the PDF morphology of the particular class under consideration. This is because all such methods of combination will propose a prob- 
abilistic output for a given pattern vector input (even if the input and/or output are ostensibly in terms of a class, as opposed to a probability, there is still an underlying PDF that may be straightforwardly reconstructed by exhaustively scanning across the pattern space). If the collective decision making process for overlapping feature sets is then more effective than that for the classifiers individually, then this is because the aggregate PDF is closer to the "true" probability density distribution. In terms of one of the more familiar combinatorial methods, the "Weighted Mean" decision system, the mechanics of PDF refinement are fairly intuitive: in this case the PDFs are combined via summing in appropriate ratio, with the final PDF recovered after a later normalizing step; and similarly, though perhaps less obviously, for the other decision schemes that have been discussed.

Thus we see that conventional combination methods, by virtue of not having specified the nature of the feature sets to which they apply, have tended to conflate two absolutely distinct methods of improving classification performance: namely, (in so far as the feature sets are distinct) classifier combination has gained its advantages by being an implicit tomographic reconstruction of the $N$-dimensional pattern space PDF, and (in so far as the feature sets are overlapping), the advantage is obtained via a refinement of the features' PDF morphology.

If we are to set about obtaining an optimal solution to the problem of classifier combination it is therefore clear that we shall have to apply these two differing mechanisms in their appropriate, and rigorously distinguished, domains of operation. That is, we should retain the classical methods of combination, but employ them only within the non-tomographic domain (to which they constitute only an imperfect approximation); that is, solely within the domain of overlapping classifiers, where they can be treated 
simply as methods of PDF refinement.

However, to return to the imperfect situation in which we are presented with overlapping features within a tomographic framework, and to consider a concrete case: suppose that there are two classifiers (A and B) that contain, respectively, the preferred feature sets $\{1,2\}$ and $\{2,3\}$ after feature selection. We then wish to obtain the best possible classification performance from the combination of features and classifiers available. There are a number possibilities open to us, for example we might:

1. Establish which of the two classifiers $\mathrm{A}$ or $\mathrm{B}$ is the better classifier of feature 2 alone, and then apply the filtered inverse Radon transformation to features 1,2 and 3 separately (feature 1 already being associated with classifier A, and feature 2 with classifier B). Note that we can envisage the first part of this as, in a sense, an implicit weighted majority vote decision scheme applied to both of the classifiers containing feature 2: this observation shall later help us to generalize our differing approaches within a unified framework.

2. Establish which of the two classifiers can least afford, in terms of classification performance, to lose feature 2, and (supposing that this is classifier A) perform the filtered inverse Radon transformation on the data sets $\mathrm{A}(1,2)$ and $\mathrm{B}(3)$ (the bracketed terms being the features associated with the classier outside the bracket). Note that there is still an implicit majority vote at the outset of this procedure, though not so obviously as in the previous case. We also note, without rigorous proof, that we might expect this to be the better option on intuitive grounds, since it does not involve either the addition of features rejected by the feature selection process (see later), or else the tomographic reconstruction of spaces that 
are already fully defined (as for 1).

3. Establish whether one of the two classifiers (A or B) is the better classifier of feature 2 alone, or whether a weighted mean combination of classifiers A and B sharing feature 2 constitutes the better classifier of that feature, and then deploy the filtered inverse Radon transformation on the three features within their designated classifiers individually. Note that we might consider this a generalization of strategy 1, permitting the two classical combination methods (majority vote and weighted mean) to vie for the representation of feature 2's PDF prior to inverse Radon transformation. We might similarly have included any of the other classical combination methods.

4. Establish which is the better classifier (either A or B) of the entire pattern space of features 1,2 and 3 and consider only that classifier's output. (An implicit weighted majority vote applied to the two classifiers' output).

5. Generate the two three-dimensional PDFs of the pattern space consisting of the features 1,2 and 3 via classifiers $\mathrm{A}$ and $\mathrm{B}$, and then combine through any of the classical methods of classifier combination. This may then be considered simply as a generalization of the preceding option. Note that we do not expect either of these possibilities to generate particularly good classifications, despite containing the full three-dimensional pattern space within the two classifiers (therefore avoiding a necessarily ambiguous tomographic reconstruction of it), because the space implicitly then includes features rejected by the feature selection process, designed, as it is, specifically to exclude those features that do not lend themselves to the generation of accurate PDFs. 
We begin to see that in the above (by no means exhaustive) list of strategies a number of consistent patterns begin to emerge. We should therefore like to generalize all of above into unified framework by giving a formal (and therefore necessarily algebraic) description of the various approaches to the problem.

Suppose, therefore, that we have a battery, T, of techniques for PDF combination excluding the Radon method, and a set of features $F_{A}$ associated with classifier $\mathrm{A}$ and a set of features $F_{B}$ associated with classifier B. Then, in the reconstruction of the pattern space $F_{A} \cup F_{B}$, we can set about generalizing the combination techniques in each of the above instances in the following manner: We firstly denote the best-performing classical PDF combination technique by ${ }^{*}\{X\}(F)$ (the star-operator extracting the optimum classifier from the total body of classifiers, X, with that classifier acting on the feature set $\mathrm{F}$ ): the converse of this, the operator that extracts the worst performing classifier we denote by $*^{\prime}\{\}$. The filtered inverse Radon combination of classifiers A, B and C containing arbitrary arrangements of non-overlapping features we shall then denote by $R_{i}[A, B, C]$. The additional functional operator ${ }^{\mathcal{F}} X$ is then introduced, which acts to extract the feature set from a particular classifying entity $X$ (whether it be a single or compound classification scheme, ie a solitary classifier or combination via conventional methods of classifiers with identical feature sets). We shall make the further assumption that the filtered inverse Radon transformation of a single PDF $R_{i}[A]$ constitutes an identity operation (ie $R_{i}[A]=A$ ), as required for algebraic consistency, and further that there exists a 'zero' of the algebra such that $\mathrm{A}($ ) (ie the classifier A acting on the empty set) produces the value "0" such that $R_{i}[X, \mathbf{0}] \equiv R_{i}[X]$.

Under the algebraic formalism we have therefore evolved the list above would be written:

1. $R_{i}\left[A\left(F_{A} \notin F_{A} \cap F_{B}\right),{ }^{*}\left\{A\left(F_{A} \cap F_{B}\right), B\left(F_{A} \cap F_{B}\right)\right\}, B\left(F_{B} \notin F_{A} \cap F_{B}\right)\right]$ 
2. $R_{i}{ }^{*} T\left\{A\left(F_{A} \notin F_{A} \cap F_{B}\right), B\left(F_{B} \notin F_{A} \cap F_{B}\right)\right\}\left(^{* \mathcal{F}} T\left\{A\left(F_{A} \notin F_{A} \cap F_{B}\right), B\left(F_{B} \notin F_{A} \cap\right.\right.\right.$

$$
\begin{aligned}
& \left.\left.\left.F_{B}\right)\right\}\right),{ }^{\prime} T\left\{A\left(F_{A} \notin F_{A} \cap F_{B}\right), B\left(F_{B} \notin F_{A} \cap F_{B}\right)\right\}\left(* ^ { \prime } \mathcal { F } T \left\{A\left(F_{A} \notin F_{A} \cap F_{B}\right), B\left(F_{B} \notin\right.\right.\right. \\
& \left.\left.\left.\left.F_{A} \cap F_{B}\right)\right\} \cup\left(F_{A} \cap F_{B}\right)\right)\right]
\end{aligned}
$$

3. as 1

4. ${ }^{*} T\left\{A\left(F_{A} \cup F_{B}\right), B\left(F_{A} \cup F_{B}\right)\right\}$

5. as 4 .

With this common framework in place, we may now seek to generalize options 1 and 2 by defining the feature sets $F_{A}^{\prime}$ and $F_{B}^{\prime}$ such that $F_{A}^{\prime} \subset F_{A}$ and $F_{B}^{\prime} \subset F_{B}$. That is, $F_{A}^{\prime}$ and $F_{B}^{\prime}$ are subsets of their respective originals permitting the empty and isomorphic sets $[\{\}$ and $\left.F_{X}\right]$ as appropriate. In conjunction with this, we further generalize the ${ }^{*}$ operator to $* O(z)$ such that it now extracts the optimal classifier with respect to every possible feature set $z$ : that is, $O(z)$ may be considered a function in its own right, although with respect to feature sets rather than classifiers as for ${ }^{*}$, and which multiplies the number of options instead of reducing them. Thus ${ }^{*} O(z)$ might be considered " $O(z)$ followed by *". This will permit us to exploit a redundancy in relation to * later on.

Within this regard, the generalization of options 1 and 2 (and therefore 3) would appear:

$$
\begin{array}{r}
R_{i}\left[A\left(F_{A} \notin^{* O\left(F_{A}^{\prime}, F_{B}^{\prime}\right) \mathcal{F}} T\left\{A\left(F_{A}^{\prime}\right), B\left(F_{B}^{\prime}\right)\right\}\right),\right. \\
\left.* O\left(F_{A}^{\prime}, F_{B}^{\prime}\right) T\left\{A\left(F_{A}^{\prime}\right), B\left(F_{B}^{\prime}\right)\right\}, B\left(F_{B} \notin^{* O\left(F_{A}^{\prime}, F_{B}^{\prime}\right) \mathcal{F}} T\left\{A\left(F_{A}^{\prime}\right), B\left(F_{B}^{\prime}\right)\right\}\right)\right]
\end{array}
$$

However, because we have specified that $R_{i}[X, \mathbf{0}] \equiv R_{i}[X]$ and, $R_{i}[X]=X$ we see that the above formulation can also be made to subsume options 4 and 5 by setting $F_{A}^{\prime}=F_{A} \cup F_{B}$ and $F_{B}^{\prime}=F_{A} \cup F_{B}$ (that is, explicitly abandoning the imposed limitation 
that $F_{A}^{\prime}$ and $F_{B}^{\prime}$ be subsets of the original feature sets), such that the above form becomes:

$$
\begin{gathered}
R_{i}\left[A\left(F_{A} \notin^{* O\left(F_{A}^{\prime}, F_{B}^{\prime}\right) \mathcal{F}} T\left\{A\left(F_{A}^{\prime}\right), B\left(F_{B}^{\prime}\right)\right\}\right),{ }^{* O\left(F_{A}^{\prime}, F_{B}^{\prime}\right)} T\{\right. \\
\left.\left.A\left(F_{A}^{\prime}\right), B\left(F_{B}^{\prime}\right)\right\}, B\left(F_{B} \notin^{* O\left(F_{A}^{\prime}, F_{B}^{\prime}\right) \mathcal{F}} T\left\{A\left(F_{A}^{\prime}\right), B\left(F_{B}^{\prime}\right)\right\}\right)\right]
\end{gathered}
$$

$$
\begin{aligned}
& =R_{i}\left[\mathbf{0}{ }^{*} T\left\{A\left(F_{A} \cup F_{B}\right), B\left(F_{A} \cup F_{B}\right)\right\}, \mathbf{0}\right] \\
& \left.=R_{i}{ }^{*} T\left\{A\left(F_{A} \cup F_{B}\right), B\left(F_{A} \cup F_{B}\right)\right\}\right] \\
& \left.={ }^{*} T\left\{A\left(F_{A} \cup F_{B}\right), B\left(F_{A} \cup F_{B}\right)\right\}\right]
\end{aligned}
$$

(equals option 4)

In equation 52 we have then obtained a very general form for the optimal strategy for dealing with overlapping feature sets, one that may be made completely general for the case of two classifiers by noting that the operator * , is in effect, a weighted majority vote combination scheme, which will therefore belong to the total body of non-tomographic combination methods, T. Hence by inverting this consideration, and applying to the above, we see that we can obtain the exhaustive combination strategy:

$$
\begin{array}{r}
R_{i}\left[A \left(F_{A} \notin^{O\left(F_{A}^{\prime}, F_{B}^{\prime}\right) \mathcal{F}} T\left\{\left\{A\left(F_{A}^{\prime}\right), B\left(F_{B}^{\prime}\right)\right\}\right),\right.\right. \\
\left.O\left(F_{A}^{\prime}, F_{B}^{\prime}\right) T\left\{A\left(F_{A}^{\prime}\right), B\left(F_{B}^{\prime}\right)\right\}, B\left(F_{B} \notin^{O\left(F_{A}^{\prime}, F_{B}^{\prime}\right) \mathcal{F}} T\left\{A\left(F_{A}^{\prime}\right), B\left(F_{B}^{\prime}\right)\right\}\right)\right]
\end{array}
$$

We might, furthermore, consider no longer restricting the overlap of feature sets to be merely one feature among two classifiers, permitting instead $\alpha$ features to overlap among $\beta$ classifiers: however we begin to see that any such process would involve a very major modification of the feature selection algorithm, upon which we are already beginning 
to encroach. Thus we can begin to appreciate that, in seeking to obtain optimality, there can be no rigorous distinction between the two processes of feature selection and classifier combination.

In fact the difficulty of overlapping feature sets that we have been seeking to address only really arises when we have been failing to rigorously distinguish between the classifier combinations that are, in effect, single classifiers, and the classifier combinations that are tomographically reconstructive in nature. We might therefore suppose that, if this distinction were built into the feature selection process, such that the final combination process were a purely tomographic procedure in relation to distinct feature sets contained within single (or classically combined) classifiers, the difficulty would never have arisen. This is indeed the case, and an optimal solution to the problem of classifier combination implemented from the level of the feature selection algorithm onwards is outlined in the following section.

\subsection{Fully General Solution to the Combination Problem: Unity of Combination and Feature Selection Processes}

To summarize our findings thus far: throughout the investigation we have found it necessary to postulate (and clarify the nature of) an apparent double aspect to the functionality of conventional classifier combination, one facet of which may be considered the refinement of PDF morphology, and therefore a form of classification in its own right, and the other being that of tomographic reconstruction, in so far as the feature sets belonging to the classifiers within the combination are distinct. Classical techniques of combination tend to conflate these two disparate aspects through not having made a 
rigorous distinction between those classifier combinations that, in effect, act as a single classifier and those combinations that may be considered to act on entirely distinct orthogonal projections of a single PDF encompassing the whole of the $N$-dimensional pattern space. We, in contrast, have found it necessary, in seeking an optimal solution to the combination problem, to make this distinction completely formal. Explicitly separating the two, however, will involve reverting to a stage prior to combination, and addressing the nature of the feature selection process itself. Thus we find we must take a unified perspective on the apparently separate issues of feature selection and classifier combination if we are to achieve our aim of attaining an optimal solution.

The essence of the unity that we are seeking will lie in ensuring that we exhaust those possibilities of classifier combination that serve only to act as single classifiers at the feature selection stage, with classifier/feature set combinations then being chosen by the feature selector only on the basis of their suitability for tomographic combination by the optimal filtered process. This basis will clearly center on the principle of supplying classifiers with distinct feature sets to the tomographic combination. The precise methodology of this procedure is therefore as follows:

Besides the classifiers $\left(a, b, c \ldots n_{c}\right)$, we must also consider as being classifiers in their own right every possible combination of these classifiers via the various non-tomographic techniques, $\left(1,2,3 \ldots n_{0}\right)$, that exist for conventional classifier combination. That is, we require the various combinations $a b_{1}, a c_{1}, \ldots ; a b_{2}, a c_{2}, \ldots ; a b c_{1}, a b d_{1} \ldots$ etc (with the appropriate combination method indicated by the numeric subscript). We must, however, also consider the possibilities of the form: $\left\{a b_{1}\right\}\left\{b c d_{2}\right\}_{3}$ (that is, the associative composition by method 3 of the pseudo-classifiers $a b_{1}$ and $b c d_{2}$ ), wherein the preceding classifier combinations may themselves be combined by any of the conventional combi- 
nation methods.

Thus the total set of classifiers of the first kind (that is to say, non-associative combinations) now numbers:

$$
\begin{aligned}
{ }^{n_{c}} C_{1}+n_{0}\left(\sum_{i=2}^{n_{c}}{ }^{n_{c}} C_{i}\right)= & { }^{n_{c}} C_{1}+n_{0}\left([1+1]^{n_{c}}-1-n_{c}\right) \\
& \quad(\text { via the binomial theorem }) \\
= & n_{c}+n_{0}\left(2^{n_{c}}-1-n_{c}\right)
\end{aligned}
$$

By a similar progression we arrive at the total number of higher order associative combinations as being (progressively): $n_{0}{ }^{n_{c}+n_{0}\left(2^{n_{c}}-1-n_{c}\right)} C_{2}+n_{0} n_{c}+n_{0}\left(2^{n_{c}}-1-n_{c}\right) C_{3}+\ldots$, giving an overall total of classifiers of both varieties of the number:

$$
\begin{aligned}
{ }^{\mathcal{P}} C_{1}+n_{0}\left(\sum_{i=2}^{\mathcal{P}}{ }^{\mathcal{P}} C_{i}\right)= & { }^{\mathcal{P}} C_{1}+n_{0}\left([1+1]^{\mathcal{P}}-1-\mathcal{P}\right) \\
& \quad(\text { via the binomial theorem }) \\
= & \mathcal{P}+n_{0}\left(2^{\mathcal{P}}-1-\mathcal{P}\right)
\end{aligned}
$$

where $\mathcal{P}=n_{c}+n_{0}\left(2^{n_{c}}-1-n_{c}\right)$

Note that, in general, there will be tautologies, and consequently simplifications, in the descriptions of the above terms: for instance $\left\{a b_{1}\right\}\left\{c d_{1}\right\}_{1}$ would be the equivalent of $a b c d_{1}$ if 1 is the (weighted) majority vote scheme. Whether it will be possible in general to exploit such redundancies for the purpose of saving computation time will depend entirely on the nature of the combination scheme.

With all of the compound classifiers that may be legitimately considered to act as single classifiers thus constructed, we may then go on, in a reverse of the usual procedure, to 
specify the appropriate feature selection algorithm. We need not in consequence worry, as we would otherwise have to if we mean to obtain an optimal solution, about selecting features for the original classifiers on the basis of their ability to act in combination (at least in conventional terms), because we have inherently constructed all of their combinations prior to feature selection: feature selection can then be conducted on a purely tomographic basis among the original classifiers and their composites.

Thus we test (exhaustively, if we require optimality) those feature combinations consisting only of distinct feature sets distributed among the classifiers and pseudo-classifiers, with final feature set selection occurring only on the basis of the features' collective ability to classify within the tomographic regime. This is illustration pictographically in figure 7 for the two combination rules, sum and product.

Hence we implicitly test all of the necessarily uncorrelated (see later) tomographically reconstructed subspaces of the $n$-dimensional pattern space against those spanned by single classifiers or pseudo-classifiers, with the maximum possible $n$-D reconstructive information being extracted from the data in consequence of this implied competition between decorrelated though stochastically-averaged, and correlated but stochasticallyvariable representations of the same probability density functions, the criterion function being the final arbiter of the outcome.

How exactly the above considerations might modify a typical, non-exhaustive feature selection algorithm, should we wish to exploit the principle within a less computationally intense framework, would depend entirely upon its nature. For instance, a sequential forward selection algorithm that might have selected feature/classifier combinations by choosing features in sequence from the total set of possibilities, allocating the selected feature to that classifier that makes the best use of it in terms of classification perfor- 
Stage 1 : Construction of composite classifiers:

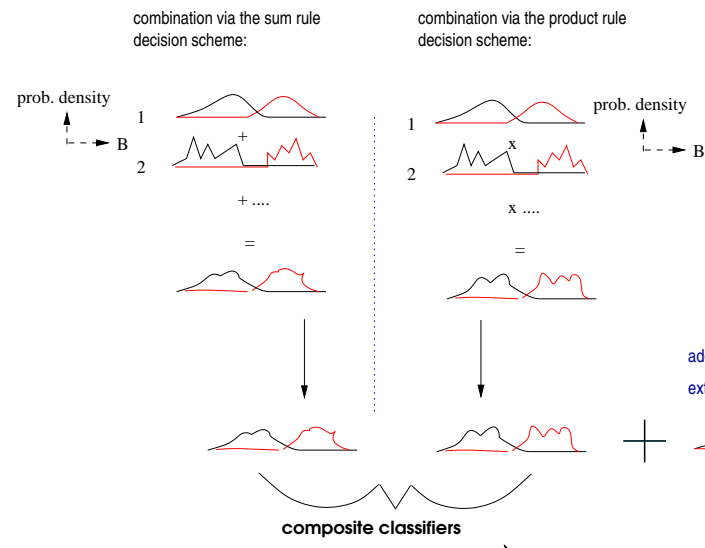

add composite classifier possibilities to original classifiers to extend range of classifier morphologies

Stage 2 : Feature Selection:

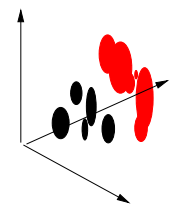

allocate DISCRETE feature sets to
above classifiers (of both types) on

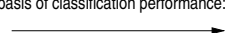

Stage 3 : Tomographically optimal combination:
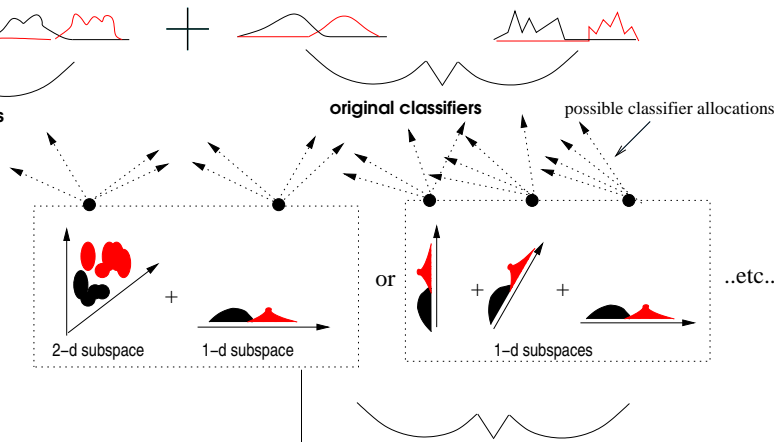

feature allocation possibilities

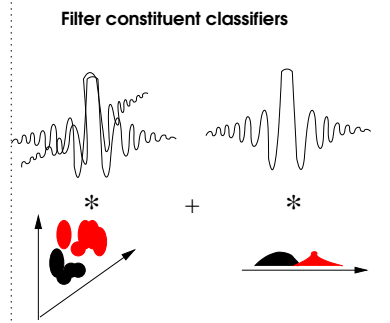

suppose this combination chosen
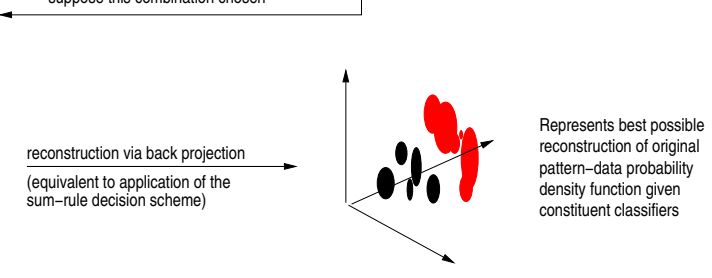

Figure 7: Unified feature-selection and tomographic classifier fusion

mance, with the processing cycle then returning to its original state, would now, under our imposed modification, become such that features are removed from the total set after they have been allocated to a particular classifier (or pseudo-classifier) prior to final tomographic reconstruction and comparison on the basis of classification performance, with no feature thus appearing more than once within the total body of feature sets, which are then hence guaranteed to be distinct. More exhaustive feature selection methods may require a correspondingly more complex modification to ensure that dis- 
tinct feature sets are obtained; however, in general, there will always exist a reasonably evident approach to achieving this.

As an additional note to conclude this section, we should also like to address the question of the optimality of the filtering procedure. In fact, a complete inversion of the tomographic reconstruction formula (equation 4) through filtration (that is, construction of an appropriate $v$ function) does not always constitute a mathematically analytic problem, the difficulty (by inspection of equation 7) being essentially one of deconvolution; a notoriously ill-posed problem in consequence of convolution being an information destroying procedure when the convolving function is bandwidth limited to any degree (or at least when more so than the data undergoing convolution). However, since we are inherently working with discrete data (due to the computational necessity of sampling the PDF at discrete intervals), this latter point does not apply to our technique, the PDF data being bandwidth limited to exactly the same extent as the filtering function. There is then no fundamental difficulty to obtaining an optimal filtering function via inversion of equation 4, merely the pragmatic one of establishing whether this can be done by analytic methods, or whether it would be better approached by numeric means. The discrete nature of the computational representation of the filter function, however, will ensure that either of the methods will suffice.

\subsection{Summary of Methodological Approach}

We have, then, in the preceding section, set out the basis for a morphologically optimal method of classifier combination via a tomographic analogy of what we now appreciate to be a major aspect of the process, the assemblage of Radon transform data, finding, in delineating this aspect, that classifier combination is inseparable from the feature 
selection process.

Our assertion of the optimality of our method then centers on it being a full completion of the partial tomographic reconstruction process implicit in all conventional methods of classifier combination, the only other considerations that we need address in this regard being, firstly, that of the remaining aspect of combination as implicit refinement of the PDF morphologies and, secondly, the robustness of the reconstructive procedure in relation to estimation error. The former point is necessarily now addressed at the level of feature selection and hence, within our unified perspective, may now be carried out at an optimal level through having distinguished it from the purely tomographic aspects of classical combination. The latter concern, the robustness of the procedure to estimation error, has been argued to be of the order of that of the Sum Rule, the previously optimal procedure in this regard, although exact calculation was omitted due to the dependence of the filtering procedure on the nature of the input PDFs.

A number practical utilizations of the outlined methodology will be given in the remaining sections of this article, the findings in relation to which will suggest that, aside from the usefulness of the knowledge of the existence of, and specification of, a theoretically optimal limit to the performance of classifier combination, the implementation of such a procedure can lead to very substantial real-world performance gains, and at a potentially small computational cost. 


\section{Post-Combination Tomographic Filtration}

\subsection{Section Introduction}

In setting out the framework of our tomographic methodology, we found it natural to specify pre-filtering of the Radon transforms as the appropriate method of removing the purely systematic morphology arising from the use of the back-projection operator. We can, however, equally well post-filter the back projection of the unfiltered Radon transforms, if it may be shown that the two methods are equivalent in terms of the resultant probability density function. Indeed, given the graphical nature of the particular tomographic process to be outlined, the latter method is in fact the more intuitive, in that we commence the key distinction of our optimal methodology, the deconvolution of the systematic morphology, at the end-point of the previously optimal fusion method (namely, the sum-rule method), with the superfluous systematic geometrical aspects of the sum fusion then being straight-forwardly inferred by visual inspection of the initial and final PDFs.

The problem of deconvolution, however, is in general an ill posed one, in that there are multiple potential solutions to the problem. We therefore generally tend to favor a specific deconvolution on a priori grounds, the very specification of which, moreover, often dictating the method of deconvolution in its entirety. Perhaps the two canonical representations of this, in the sense that they collectively represent the extremes of the gamut of possibilities, are the maximum entropy (cf eg [16]) and the Högbom [9] algorithms, which respectively presume the piecewise continuity (strictly, minimum information-theoretic complexity), and the discreteness, of the final solution.

Because of the unique form of our tomographic problem, however, we shall find it useful 
to specify our own method of deconvolution, based only on the one a priori assumption, namely that any deconvolutional ambiguity that gives rise to a choice between imposing an arbitrary axial asymmetry on the final PDF and one that does not, then the latter alternative will always be the favored one. This is equivalent to insisting that, in the absence of any information to the contrary, the feature axes will inherently constitute a favored orientation in relation to the data, taking precedence over any of the other potential oriental axes. Such an imposition amounts, in fact, to the specification of a fully decorrelated reconstructive space with regard to the constituent features, and is actually the default outcome of the pre-filtering approach, thus rendering the a priori stance in relation to post-filtering a fully necessitated one, given their equivalence.

We thus set about implementing this consideration within the context of a modified version of the Högbom algorithm; that is: a Högbom algorithm to the degree that it involves the recursive subtraction of infinitesimal simulacra of the systematic artifacts (the "blurring" function of equation 7) from the back-projected (sum rule) data: modified to the extent that when the algorithm is required to subtract several systematic simulacra such that their overlap could themselves be interpreted as geometric simulacra (in which case there is an ambiguity as to the distinction between "overlap" and "simulacra"), then all of these correlated entities are treated as equally indicative of the underlying "de-blurred" morphology. Thus, in essence, we implement a recursive Högbom deconvolution algorithm with an additional intermediate stage mapping the various correlations between the proposed subtractions to ensure that the artificially imposed and unrepresentative dichotomy between the subtractions and their intersections does not impact on the final recovered PDF.

In broad terms, then, this process is implemented in the following manner: 
In carrying out an explicitly two-dimensional implementation of the mathematical stages that give rise to equation 29, we found that the unfiltered 2D inverse Radon transformation equates to:

$$
P(x, y)_{\text {recovered }}=P_{1}(x)+P_{2}(y)
$$

for the two classifier density functions $P_{1}(x)$ and $P_{2}(y)$, distributed respectively over the variables $x$ and $y$ (ignoring normalization considerations).

Now, we also have from equation 7 that the unfiltered inverse radon transform is equal to:

$$
f_{\text {recovered }}=R^{*}\left(f_{\text {original }}\right)=f_{\text {original }} * B(x, y)
$$

with $B(x, y)$ the "blurring function" or systematic artifact. Therefore if we set $f_{\text {original }}$ to be the delta function $\delta\left(x_{1}, y_{1}\right)$, such that $f_{\text {recovered }}=B\left(x-x_{1}, y-y_{1}\right)$, then we have that:

$$
P_{1}(x)+P_{2}(y)=B\left(x-x_{1}, y-y_{1}\right)
$$

On the assumption that $P_{1}(x)$ and $P_{2}(y)$ are representative of their respective projections of the two-dimensional PDF: $f_{\text {original }}=\delta\left(x_{1}, y_{1}\right)$, such that equation 1 holds; ie $P_{1}(x)=$ $\delta\left(x-x_{1}\right)$ and $P_{2}(y)=\delta\left(y-y_{1}\right)$, we then obtain the equivalence:

$$
B\left(x-x_{1}, y-y_{1}\right)=\delta\left(x-x_{1}\right)+\delta\left(y-y_{1}\right)
$$


Removing the position dependences $x_{1}$ and $y_{1}$ by coordinate transformations this gives the pure blurring artifact to be the cross-shaped function:

$$
B(x, y)=\delta(x)+\delta(y)
$$

Our modified Högbom algorithm therefore involves the recursive subtraction of an infinitesimal version of this artifact, shifted appropriately, from all discrete two-dimensional PDF values (recovered via back projection) within some small fixed percentage of the maximum value. A scalar quantity proportional to the infinitesimal magnitude of this value (which we denote $C$ ) is then added to the existing (initially zero) quantity associated with the value's coordinates. This latter matrix will then constitute the proposed PDF deconvolution at the termination of the procedure, which occurs when the initial matrix first generates negative values on subtraction of the infinitesimal blurring artifact. The feature specific to our post-filtering approach, namely the a priori assumption of the priority of the feature axes becomes apparent in the particular way in which we deal with the (almost ubiquitous) situation in which multiple infinitesimal blurring artifacts are to be simultaneously subtracted from the data. As we have indicated, there is an ambiguity as to what constitutes an artifact and what constitutes the overlap of an artifact: we see from an inspection of the form of $B(x, y)$ that the proposed subtraction of artifacts centered at (say) $\left(x_{a}, y_{a}\right)$ and $\left(x_{b}, y_{b}\right)$ would lead to a double subtraction of the infinitesimal value $C$ from the points $\left(x_{a}, y_{a}\right),\left(x_{a}, y_{b}\right),\left(x_{b}, y_{a}\right)$ and $\left(x_{b}, y_{b}\right)$, without a corresponding registration of the points $\left(x_{a}, y_{b}\right)$ and $\left(x_{b}, y_{a}\right)$ in the final deconvolution matrix. This occurs because the intersection of two blurring artifacts has itself the precise form of a blurring artifact, and the claim to primacy of the original arti- 
facts over the intersecting region is thus invalidated. Thus, we must seek to correlate all of the proposed subtractions with each other, in order to establish whether there exist any overlapping regions that might themselves have to be considered constitutive of subtractive entities and consequently registered alongside the originals in the final deconvolution matrix.

It is thus clear that any such post-filtration methodology must invariably involve the nesting of a series of complex conditional tests, and, hence, require considerable computational expenditure. It will consequently be the endeavor of the following subsection to reconfigure the proposed post-filtration system to achieve this on feasible time-scales.

\subsection{An Economic Approach to Post-Combination Tomographic}

\section{Filtration}

We have thus argued that the most straightforward approach to removing the possibility of negative PDF values in the tomographically reconstructed feature-space is that of unfiltered (post-)deconvolution, via an adaptation of the Högbom deconvolution algorithm [9]. The iterative nature of this technique allows a piece-by-piece removal of systematic artifacts, such that in its unmodified and mathematically ideal form, the procedure can be considered to impose an a priori condition of least possible correspondence of the recovered morphology to the feature axis geometry [10]. Thus, the procedure embodies a distinct methodology for distinguishing between the degenerate solutions that all methods of deconvolution must address whenever there exist zeros in the Fourier transform of the entity to be deconvolved. Moreover, it invariably generates positive-definite solutions. 
Endeavoring to reduce the computation time involved in this procedure will involve establishing the degenerate form of that tomographic reconstruction adopts under the particular geometric constraints of pattern-space reconstruction. In quantitative terms, this will permit a reduction in the number of computational cycles required to execute the procedure from $\sim X^{3 n-1}$ to $\sim X^{n-1}$, $(n$ being the dimensionality of the problem and $X$ its sampling resolution).

This three orders of magnitude reduction in the computational complexity of the problem brings the tomographic method well within the realms of practical feasibility, as well as giving a more intuitive description of the process in graphical terms, perhaps serving as a backdrop for future extensions of the technique.

\subsection{Nature of Högbom Deconvolution in the Sum-Rule Do- main}

Following on from the above illustration, throughout the following two sub-sections the discussion of Högbom deconvolution in the Sum-Rule domain shall be confined to a two-dimensional reconstructive space (that is to say, with two single-featured PDFs constituting the classifiers in the combination) for reasons, again, of conceptual simplicity as well as ready graphical perspicuity. That the generic results derived for this space are straightforwardly generalizable to an arbitrarily-dimensioned reconstructive space (one consisting of an arbitrarily large set of constituent classifiers of unconstrained featurespace dimension) follows from the recursive argument for progressively building up the reconstructive PDF space set out in section 2.2.

It was hence established in the preceding introduction to post-filtration methods that 


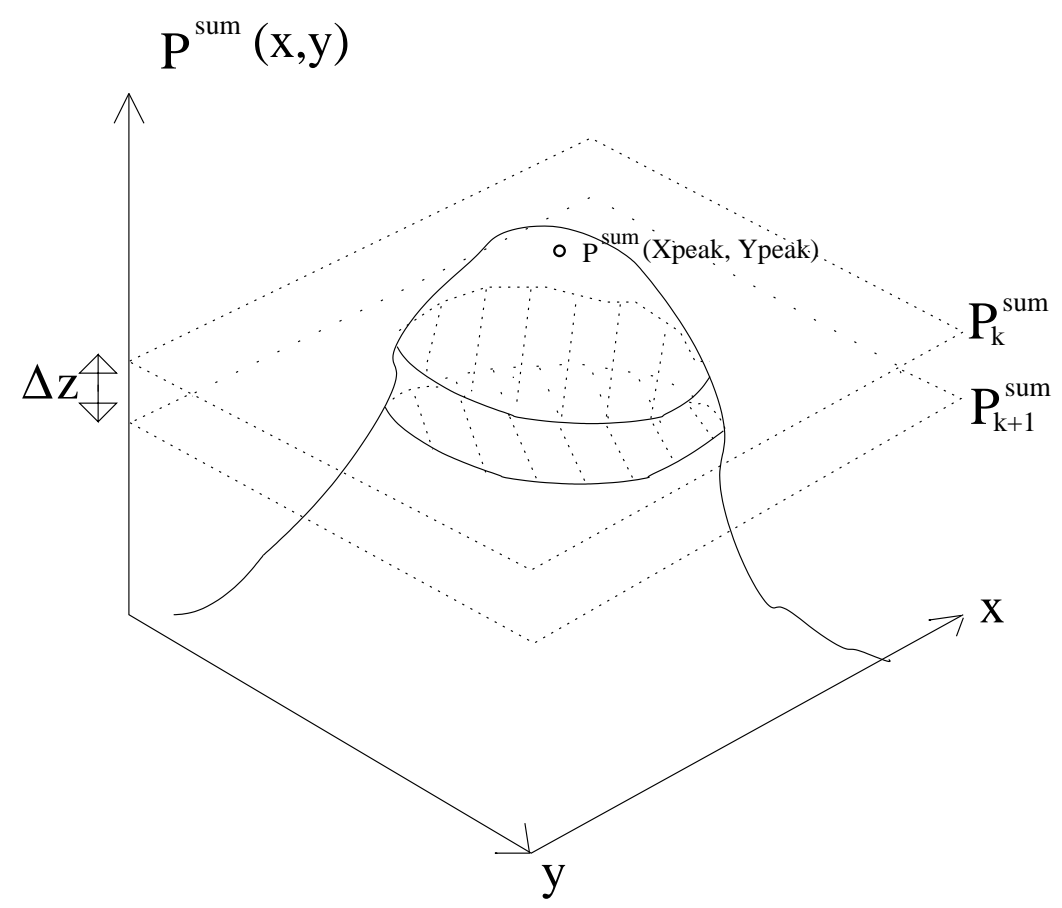

Figure 8: The composite PDF in the Sum-Rule space

the Sum-Rule space post-convolution artifact for two-feature dimensions is equivalent to a "cross" of infinitesimal width (ie $P_{\text {artifact }}(x, y)=\delta(x)+\delta(y)$ ). It is consequently this entity (modified appropriately to account for the discrete sampling of the PDF inherent in a computational methodology) that we are seeking to remove via recursive Högbom subtraction. In the two-dimensional case we have specified (see fig. 8) this occurs as follows: a counter value, $z$, is set at the peak value of the Sum-Rule space, with a recursive scanning cycle then initiated to establish the set of all positions within a probability density value $|<\delta z|$ below this. After registration of these points in a deconvolution matrix (so called because it will ultimately constitute the proposed deconvolution), through adding a value $\delta z$ to any existing value at the designated coordinates, a set of cross-artifacts centered on those points are then subtracted consecutively across the space. This process is repeated until a subtraction is proposed that would yield negative values in the Sum-Rule space, with a complete deconvolution therefore resulting in a 
residual-free (ie zero-valued) space. (Note that in the application to astronomical data, the procedure must rely instead on a stochastic criterion of completion in the absence of an absolute zero-point, namely the indistinguishability of the histogram of point values from a Poissonion "noise" distribution). The terminal point of the procedure therefore invariably represents (even in the absence of a proper termination) a positive-definite solution in the deconvolution matrix, as demanded by probability theory. This procedure will be more fully quantified at the computational level in the following section, however, we must first address a significant difficulty that arises with this approach:

\subsubsection{Finite Sampling Issues}

It is immediately apparent in any computational implementation of the Högbom deconvolution algorithm in the tomographic domain (and to a significantly lesser degree, in the astronomical domain) that the issue of the necessarily finite setting of the value $\delta z$ becomes non-trivial. It is intuitively obvious that the process achieves mathematical ideality only in the asymptotic limit: $\delta z \rightarrow 0$, in which case each iterative stage registers an unambiguous set of discrete points at uniform height. However, the fact that any computational implementation must rely on a finite value of $\delta z$ gives rise to complications that have consequences that go far beyond issues of sampling accuracy: selecting different values of $\delta z$ for the situation set out above in fact generates vastly divergent sets of convergences at the termination of the procedure. We can illustrate this point via the following scenario.

Consider a conceivable (as well as an apparently eminently deconvolvable) Sum-Rule space consisting of four Dirac delta cross-function artifacts scaled to have integral values in the range $4 \delta z \rightarrow 6 \delta z$, and centered on the the respective positions $\left(x_{0}, y_{0}\right),\left(-x_{0}, y_{0}\right),\left(-x_{0},-y_{0}\right)$ 
and $\left(x_{0},-y_{0}\right)$ (the functions being broadened sufficiently to account for the discrete sampling of the space by convolution with the spatial sampling element). Such a space may be constructed via two constitutive probability density functions consisting of (for example):

$$
P(x)=(2 \delta z+3 \delta z / 4) \delta\left(x-x_{0}\right)+2 \delta z \delta\left(x+x_{0}\right)
$$

and

$$
P(y)=(2 \delta z+3 \delta z / 4) \delta\left(y-y_{0}\right)+2 \delta z \delta\left(y+y_{0}\right)
$$

The value $6 \delta z$ would then represent a "cut-off" value above which the Högbom algorithm has already subtracted components. The first new pass of the Högbom algorithm over the remaining configuration would then result in the registration of the point $\left(x_{0}, y_{0}\right)$, with a probability density $(5+1 / 2)$, and a value $\delta z$ entered in the deconvolution matrix: the removal of the corresponding cross function; $\delta z\left(\delta\left(x-x_{0}\right)+\delta\left(y-y_{0}\right)\right)$ subtracts a value $\delta z$ from (among the other points) the Dirac delta cross-functions centered on $\left(-x_{0}, y_{0}\right)$ and $\left(x_{0},-y_{0}\right)$. The value $P^{s u m}\left(x_{0}, y_{0}\right)$ is evidently then decimated by $2 \delta z$, leaving the only point of $P^{\text {sum }}(x, y)$ within the range of the next iteration of the Högbom algorithm (ie $4 \delta z \rightarrow 5 \delta z)$ as $\left(-x_{0},-y_{0}\right)$, with a value of $4 \delta z$. The iteration thus repeatedly shuttles back between registration of, and subtraction at, the points $\left(-x_{0},-y_{0}\right)$ and $\left(x_{0}, y_{0}\right)$ until the procedure reaches a termination via the criteria discussed earlier. The significant aspect of this operation is that the values associated with the points $\left(x_{0},-y_{0}\right)$ and $\left(-x_{0}, y_{0}\right)$ are repeatedly subtracted from in such a fashion as to never be registered, and hence the deconvolved space consists only of delta functions centered on the points 
$\left(-x_{0},-y_{0}\right)$ and $\left(x_{0}, y_{0}\right)$. It should, however, be noted that this terminal point does represent a consistent deconvolution given the initial data, in the sense that the recovered distribution, if re-convolved by the cross-artifact, would indeed revert to the originally specified Sum-Rule space.

If, however, we now set $\delta z$ to three-quarters of its former value, the progression of registered points follows instead a sequence whereby the point $P^{s u m}\left(x_{0}, y_{0}\right)$ is again initially registered: this time, though, the subtraction of the cross-artifact (being now proportional to $\left.\frac{3}{4} \delta z\right)$ leaves all four of the above points; $\left(-x_{0},-y_{0}\right),\left(x_{0},-y_{0}\right),\left(-x_{0}, y_{0}\right)$ and $\left(x_{0}, y_{0}\right)$, at the same value $(4 \delta z)$. Any subtractions occurring after this point must invariably be fully symmetric, that is, involving all of the four points simultaneously. Hence, the final recovered deconvolution would consist of three Dirac delta-functions of equal height centered on the points $\left(-x_{0},-y_{0}\right),\left(x_{0},-y_{0}\right)$ and $\left(-x_{0}, y_{0}\right)$, with a forth, higher, delta-function centered on $\left(x_{0}, y_{0}\right)$.

Note that this, like the last example, is also a consistent deconvolution given the initial data. It would therefore seem that the method, unlike the majority of iterative deconvolution techniques, does not in fact guarantee convergence on a unique solution, in the sense of a requirement that the accuracy of the final solution be only proportionately sensitive to methodological parameters like step size (this does not, however, preclude an a priori assumption of the asymptotic limit at $\delta z=0$ as being, in some sense, the "most accurate" solution). This is consistent with earlier astronomical findings in relation to the Högbom method [10], namely, that it does not delineate any known criterion of solution accuracy that could constitute a linear "distance" measure between the putative solutions and the ideal case. There is therefore no mechanism for ensuring that each iteration represents a progressive convergence towards some optimal, if only hypothet- 
ically existent solution (indeed, this is generally how such iterative methods operate by progressively subtracting error residuals from the input data). As such, the Högbom technique cannot be relied upon to suppress divergences at the iterative level.

This might constitute a serious conceptual impediment to the methodology, were it not for the fact that the Sum-Rule space, being tomographically constructed from lowerdimensional constituent classifier PDFs, is incapable of containing any information that could be 'lost' or represented incorrectly through an in-apposite favoring of any particular one of the proposed solutions of the Högbom method, such as might be the case if we commenced with a completely unconstrained space. So long as making a particular selection between the various possibilities accurately reflects some conceptually justified a priori consideration, the lack of a fixed accuracy criterion for the final solution does not, therefore, represent any significant impediment. The most appropriate such approach is in fact the previously stated one, the favoring of the $\delta z=0$ solution, on $a$ priori grounds that we shall set out more fully later.

It remains, however, to implement this within a discretely-sampled computational setting: having thus established, though, that there is not an inherent ambiguity of deconvolution utilizing the Högbom methodology within a tomographic context, our difficulty is then purely operational, and most obviously mitigated by discrete sampling of the probability density axis, and setting $\delta z$ to be an exact divisor of this sample width (as was implicitly carried out in [17]). Such an approach, it may be intuitively seen, will guarantee an unambiguous convergence on a solution approximating that of $\delta z=0$, to within a set of bounds defined by their proportionality to that sample width, consistent with our earlier requirements.

Needless to say, this micro-sampling of an already minutely sampled PDF distribution 
does not lend itself to efficient computation, particularly as, for optimal classification performance, it must be repeated at each pass of the feature selection algorithm [17]. A far more effective approach is therefore that proposed in the following section, which does not require this sampling step, and which readily lends itself both to further computational, and more significantly, further algorithmic, compaction.

\subsection{Efficient Implementation of Högbom Deconvolution in the PDF Domain}

In proposing a finite $\delta z$ procedure that does not experience the above problem (that is to say, whose solution has no explicit dependence upon the value of $\delta z$ ), we have to consider more systematically what is taking place during the simultaneous subtraction of cross-artifacts implicit in each iteration. As is uniquely the case for tomographic reconstruction of a pattern-space, these subtraction entities share an identity with the form of the axial system (that is to say, constitute a complex of intersecting rectanguloids of varying dimensionality). We can therefore appreciate that the simultaneity of the subtraction immediately gives rise to the irreconcilable ambiguity mentioned in the section introduction: we see that the overlap of these entities necessarily gives rise to further intersections at specific points of the pattern space, the artifacts around which are of the same form as the axial system, which are hence not in any real sense distinguishable from the original points at which axial artifacts are subtracted.

It was therefore considered apposite to propose as a modification of the Högbom method in the expert fusion domain, that those additional points be put forward as candidates for registration alongside the originals. It is, in a sense, therefore possible to regard 
this modification as summing over all possible deconvolution solutions that we earlier encountered at the iterative level. This amounts to applying the most conservative criterion of PDF correlation within the terms of the Högbom approach, while maintaining the most presumptive a priori condition on the feature correlation in more general terms (which is to say, imposing an assumption of maximal feature interdependence, the alternative having been eliminated at the feature selection stage).

It is most useful, in visualizing this alternative approach, to focus on the effect of the Högbom algorithm on the PDFs constituting the combination, rather than the on the Sum-Rule space, as we have hitherto done. The nature of the Högbom iteration is also rendered far more graphically evident from this perspective:

\subsubsection{PDF-Centered Approach}
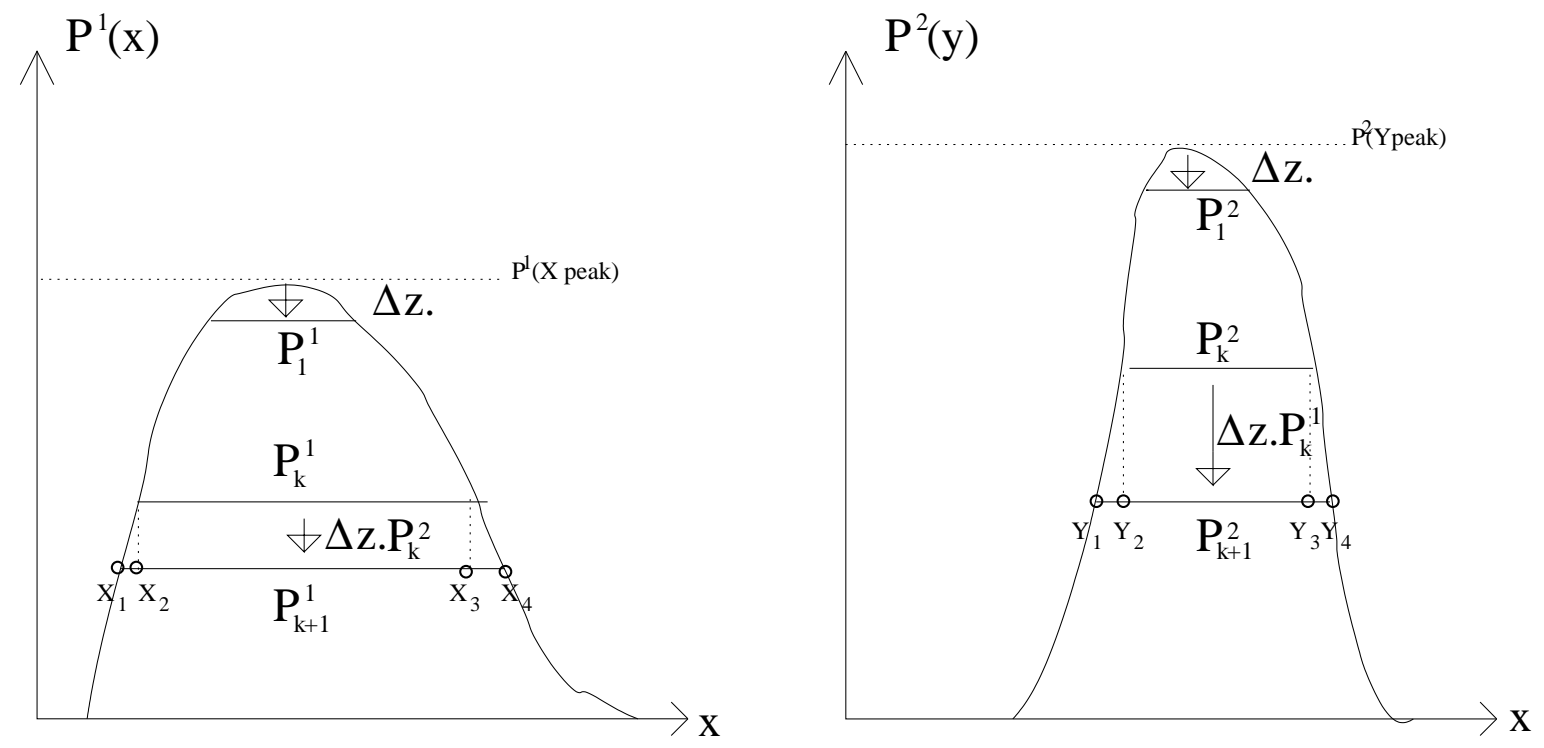

Figure 9: Requisite subtractions from the 2 PDFs constituting the combination in the modified methodology

As we indicated earlier, then, the commencement of the Högbom procedure consists in establishing the peak position of the Sum-Rule space $\left(P^{\text {sum }}\left(X_{\text {peak }}, Y_{\text {peak }}\right)\right.$ from fig. 
8), and deriving the set of points, $P_{1}^{\text {sum }}$, that lies in the probability density range $\left(P^{s u m}\left(X_{\text {peak }}, Y_{\text {peak }}\right) \rightarrow P^{s u m}\left(X_{\text {peak }}, Y_{\text {peak }}\right)-\delta z\right)$ prior to a subtraction of the crossartifacts centered on those points. We should now like to associate these points with particular sets of ordinates in the PDF domain such that it is possible to view the 3dimensional process of fig. 8 within the 2-dimensional format of fig. 9 . This would not in general be possible to do in a straightforward fashion if the subtraction entity were of an arbitrary form. However, the fact that the subtraction artifact mirrors the axial system means that it may be equivalently represented as the independent summation of 1-dimensional Dirac delta functions (convolved by the sampling element $\delta x$ ) centered on the appropriate ordinates of the $\underline{\mathrm{PDF} \text { domain. }}$ The process of subtraction of a single artifact in this domain therefore acquires the intuitive aspect of a subtraction of the two delta functions from the appropriate points of the respective PDFs $\left(\delta\left(x-x_{0}\right) \delta x\right.$ from $P^{1}(x)$, and $\delta\left(y-y_{0}\right) \delta y$ from $\left.P^{2}(y)\right)$

Although this situation readily generalizes to the arbitrarily-dimensioned case, it becomes somewhat more complex for multiple subtractions of the type indicated earlier, in that the subtraction of cross-artifacts centered on the additional set of points created by the intersections of the artifacts (arising from the originally detected points) leads to an asymmetry in the corresponding PDF domain subtractions: the particular value to be subtracted from each of the ordinals in a particular PDF turns out to require a proportionality to the subtractions in the remaining PDFs constituting the combination. This is illustrated in fig. 9 for a mid-point of the deconvolution's execution (since we are required to externally impose an infinitesimal subtraction on the first iteration of the sequence $k=1,2 \ldots$, which cannot, therefore, exhibit this effect explicitly).

A subtraction, then, of the points above $P_{k+1}^{s u m}$ (points above $P_{k}^{s u m}$ having been assumed 
to have been removed by previous iterations) leads to a mapping of the ordinal sets: $P_{k}^{1} \rightarrow P_{k+1}^{1}$ and $P_{k}^{2} \rightarrow P_{k+1}^{2}$ : that is to say, a reduction of $\delta z\left|P_{k}^{2}\right|$ and $\delta z\left|P_{k}^{1}\right|$ in $P_{k}^{1}(x)$ and $P_{k}^{2}(y)$, respectively (with a corresponding registration of $\delta z$ in the deconvolution matrix for the point-set $\left(X_{1} \rightarrow X_{2}, Y_{1} \rightarrow Y_{2}\right)$, that is, all combinations of ordinals over this range). Note in particular the transfer of width information from one PDF to the other, giving rise to the mutually morphologically dependent convergence alluded to earlier: we are then now implicitly regarding the PDFs, not as maps $\mathcal{R} \rightarrow \mathcal{R}$, but rather as morphological entities delineating 'areas' in an ordinate-probability space.

The fact that these points lie in bands is critical to the method's economy, and a consequence both of the explicit inclusion of the intersection point-sets (of which more later), but also of the particular nature of this stage-by-stage re-mapping. For the set of ordinates newly incorporated into the $(k+1)^{\prime}$ th iteration to be consistent with the line defined by the ordinate set arising from the $k^{\prime}$ th iteration, this involves imposing a map:

$$
\begin{gathered}
P_{x}^{1} \rightarrow P_{X_{1}}^{1} \quad \forall X_{1}<x<X_{2} \text { and } X_{3}<x<X_{4} \\
P_{y}^{2} \rightarrow P_{Y_{1}}^{2} \quad \forall Y_{1}<y<Y_{2} \text { and } Y_{3}<y<Y_{4},
\end{gathered}
$$

at each new stage of the process, such that each new ordinate set is contained within its predecessor. Thus, the algorithmic recursion applies solely now to these ordinal sets (two single-dimensional entities, rather than to a single Sum-Rule density function of three dimensions). It should also be noted that this approach is equally valid for the more complex case of multiply-peaked PDFs, the extension to the mapping protocol 
being self-evident.

The other issue which we have yet to approach systematically that arises in relation to multiple subtractions within this framework is that of the cross-correlation between subtractive entities. In fact, it transpires that a quantitative treatment of this effect is rendered significantly more straightforward on consideration within the PDF domain: in removing multiple delta-function elements from the individual density functions, all of the interstitial "overlap" artifacts are implicitly dealt with at the same time.

This can be illustrated in the 2-dimensional case via an appreciation that the subtraction of delta-function elements centered on the $P^{1}$ ordinals $X_{1} \& X_{2}$, and the $P^{2}$ ordinals $P^{2}\left(Y_{1}\right) \& P^{2}\left(Y_{1}\right)$, would imply a subtraction of cross artifacts centered on $P^{s u m}\left(X_{1}, Y_{1}\right)$, $P^{\text {sum }}\left(X_{2}, Y_{1}\right), P^{\text {sum }}\left(X_{2}, Y_{2}\right)$ and $P^{\text {sum }}\left(X_{1}, Y_{2}\right)$ : that is to say, the complete set of conceivably detectable points in the Sum-Rule domain and their subtraction-artifact overlaps. The only remaining issue to address in relation to the PDF-centered approach to Högbom deconvolution is then the construction of the actual coordinates for registration in the deconvolution matrix. This, it is readily appreciated, is just the set of all permutations of detected ordinals in the current iteration, and may be constructed accordingly.

In this manner, by switching to a PDF-oriented approach, necessitating what is effectively a varying $\delta z$ methodology within which the issue of multiple registrations and subtractions is dealt with automatically, we have effectively dissolved the distinction between point-detection, artifact-correlation and artifact subtraction. Thus, we have generated a very significant speed increase through the fusion of the three space-scanning processes implicit in their original division, and a further, arbitrarily large speed increase determined by the implicit fusion of the $\delta z$ parameter with the morphology of 
the PDFs via the inclusion of cross-sectional magnitude terms within each iteration (although note that this term will in general be dependent on the dimensionality of the constituent classifier feature spaces). We shall determine more precisely the effect that this has on the computational efficiency in the following sub-section:

\subsubsection{Algorithmic Implementation}

Having thus set out a revised framework for Högbom deconvolution, within which permitting a varying $\delta z$ acts to resolve the ambiguity in the solution convergences, and also to permit a range of algorithmic economies to be made, we are finally in a position to specify what the corresponding programmatic efficiency gains might be.

The first such economy, arising as a consequence of the implicit identification of the peak-search, peak-correlation and artifact-subtraction procedures, reduces a process of originally $\sim[X]^{2 n}\left[X^{n-1}+X\right]$ cycles to around $X^{n-1}$ cycles $(n$ being the dimension of the reconstructive space, and $X$ its linear sampling resolution: the square brackets denote a maximum value). This comes about in the following way: within the unmodified Högbom procedure each iterative scan of the Sum-Rule space to obtain a set of points for subtraction carries with it a penalty of $X^{n}$ cycles. Because $\delta z$ is not correlated with the PDF cross-sections as it is in the modified case, the requisite analysis of subtractionartifact overlapping will require that the additional interstitial points are all individually constructed and registered within the deconvolution matrix. In the worst case scenario, when the ordinates of the detected points cover the entirety of the feature axes, this would amount to an implicit scan over the entire reconstructive space, requiring an additional computation of $[X]^{n}$ cycles (a scan being effectively the exhaustive cyclic construction of ordinal permutations). 
A deconvolution-artifact subtraction at each of these points would then require a further scanning agent to act over the reconstructive space, ostensibly involving a further $X^{n}$ cycles per point. However, it is possible to break the artifacts down into their constituent iterations to obtain a reduction in this. That is, if the set of classifiers constituting the combination have an individual feature-dimensionality given by: $\left(d_{1}, d_{2}, d_{3} \ldots\right)$, then this would represent a required per-point cycle count of $\left(X^{d_{1}}+X^{d_{2}}+X^{d_{3}} \ldots\right)$ in order to perform the subtraction. In execution terms, this represents a maximum of $X^{n-1}+X$ cycles (the best case scenario being just $n X$ cycles, or $2 X$ in our example). The total cycle count per iteration for the Högbom method is therefore written: $X^{n}[X]^{n}\left[X^{n-1}+X\right]$. In contrast, the proposed alternative, in combining the detection, correlation and subtraction procedures, permits a minimum cycle count of only $X^{n}$ per iteration. This comes about through combining the activity of a detection/subtraction scan over just the constituent PDF feature dimensions (which would in itself now carry only a $\left[X^{n-1}+X\right]$ cycle penalty) with a correlation analysis (which would normally constitute an additional $[X]^{d_{1}}+[X]^{d_{2}}+[X]^{d_{3}} \ldots=[X]^{n}$ cycles per point), such that the correlation analysis, in generating every possibly ordinal permutation, now implicitly performs the detection and subtraction operations in the manner described earlier.

It is possible to yet further improve on this performance for the particular case of the constituent classifiers constituting point-wise continuous PDFs, through the introduction of a second-order programmatic economy deriving from the modified format. We note in fig. 9 that $P_{k}^{1}$ is fully contained within the set $P_{k+1}^{1}$, with only the sets $P^{1}\left(X_{1}\right) \rightarrow P^{1}\left(X_{2}\right)$ and $P^{1}\left(Y_{1}\right) \rightarrow P^{1}\left(Y_{2}\right)$ then contributing a new behavioral aspect to the $(k+1)$ 'th iteration (and similarly for $P^{2}(Y)$ ). Thus, the newly correlated and registered points in the $(k+1)$ 'th iteration will all lie $\underline{\text { inside }}$ of the region $P^{s u m}\left(X_{1} \rightarrow X_{4}, Y_{1} \rightarrow Y_{4}\right)$ and 
outside of the smaller region $P^{s u m}\left(X_{2} \rightarrow X_{3}, Y_{2} \rightarrow Y_{3}\right)$. Hence, and this is equally true for multiply-peaked PDFs, it becomes possible to simply discard this region within the correlation analysis (by far the most computationally expensive part of the modified procedure) leaving only the originally specified artifact subtraction to perform at a penalty of $\left[X^{n-1}+X\right]$ cycles. In algebraic terms this results in a cycle count reduction to

$$
\begin{aligned}
& \left\{\left[X^{n-1}+X\right]\right\}+\left\{(X+d x)^{n}-X^{n}\right\} \\
& \approx\left\{\left[X^{n-1}+X\right]\right\}+\left\{n d x X^{n-1}\right\}
\end{aligned}
$$

(the later bracketed term in the addition being the generalization of this reasoning to arbitrary dimensionality: $d x$ is the sampling element, of order $\delta z$ ). This is clearly, then, a very substantial additional saving.

As a final note, it is evident that the number of iterations is itself a governor of execution time and, as we have observed, need not necessarily be fixed, a fact from which we have gained considerable advantage. However, the actual value of the number of iterations is governed by morphology, and is consequently not straightforwardly enumerable. The original Högbom method, however, does not suffer this limitation, requiring $\left(P_{\max }^{1}+\right.$ $\left.P_{\max }^{2}+\ldots\right) / \delta z$ iterations to execute, and may thus serve as a maximum limit for the modified procedure (though in practice we would expect the true value to be a small fraction of this).

Thus, in the final analysis, the total cycle count for the more efficient methodology can be written: 


$$
\begin{aligned}
& {\left[\left(P_{\max }^{1}+P_{\max }^{2}+\ldots\right) / \delta z\right]\left\{\left[X^{n-1}+X\right]\right\}+\left\{n d x X^{n-1}\right\}} \\
& \approx\left[\left(P_{\max }^{1}+P_{\max }^{2}+\ldots\right)\right]\left\{\left[X^{n-1}+X\right] / \delta z\right\}+\left\{n X^{n-1}\right\}
\end{aligned}
$$

as opposed to:

$$
\left(P_{\max }^{1}+P_{\max }^{2}+\ldots\right) / \delta z\left\{X^{n}[X]^{n}\left[X^{n-1}+X\right]\right\}
$$

under the original proposal.

A pseudo-code implementation might be given as follows:

\subsubsection{Step-by-Step Approach to Procedurally Implementing Performance- Optimized 'Filtered Back-projection'}

1. Assemble experts constituting the combination as a series of PDFs ranging over $n$ discrete feature spaces of respective dimensionality; $a_{1}, a_{2} \ldots a_{n}$ for the class set; $\omega_{1}, \omega_{2}, \ldots \omega_{m}$

2. Select the first class of the series, $\omega_{1}$, and establish peak probability density value(s), $P_{a_{i}}$, for of each expert's individual representation of that class.

3. Specify a pair of accuracy parameters, $d z$ and $d x$, that respectively denote the probability density and feature-space resolutions.

4. Establish the 'hyper-area' between the probability density ordinates representing the peak value and (peak value-dz) for each of the classifier PDFs: ie, the scalar number of $(d x)^{a_{i}} \times d z$ units between the two probability density values for each of the classifiers in the fusion. 
5. Specify a matrix of dimension; $a_{1}+a_{2}+\ldots+a_{n}$ with each element designating an (initially zero) probability density value attributable to every $(d x)^{a_{1}+a_{2}+\ldots+a_{n}}$ unit of the composite feature-space. ${ }^{2}$ Add a value, $N$, to those points representing all combinations of $n$ concatenations of the respective (co-)ordinates established in 4 : That is, the Cartesian product $\left\{\vec{X}_{1}\right\} \times\left\{\vec{X}_{2}\right\} \times\left\{\vec{X}_{3}\right\} \times \ldots \times\left\{\vec{X}_{n}\right\}$. (N must be $\left.>\sum_{i=1}^{n} P_{a_{i}}\right)$

6. Subtract the resolution parameter $d z$ from each peak value $P_{a_{i}} ; \forall i$, and set an iteration parameter (say, $t$ ) to zero.

7. Subtract a quantity $\left|X_{1}\right| \times\left|X_{2}\right| \ldots \times\left|X_{i-1}\right| \times\left|X_{i+1}\right| \times \ldots \times\left|X_{n}\right| \times d z$ from the current peak value of each classifier, $P_{a_{i}} ;\left|X_{j}\right|$ being the scalar values derived in $\mathbf{5}$, ie: the number of coordinate vectors $\left\{\vec{X}_{i}\right\}$ of dimensionality $a_{i}$ counted by the PDF hyper-area establishing procedure above. Note, especially, the absence of $\left|X_{i}\right|$ in the product entity.

8. Establish the new hyper-area value associated with the subtraction 7 , that is: the hyper-area between the probability density ordinates representing the previous and current peak-values (as per 4).

9. Allocate a value $N-t . d z$ to those points in the deconvolution matrix representing novel coordinates established after the manner of 4 . That is, the Cartesian product difference:

$\left[\left(\left\{\vec{X}_{1}\right\}_{\text {old }} \cup\left\{\vec{X}_{1}\right\}_{\text {new }}\right) \times\left(\left\{\vec{X}_{2}\right\}_{\text {old }} \cup\left\{\vec{X}_{2}\right\}_{\text {new }}\right) \times \ldots \times\left(\left\{\vec{X}_{n}\right\}_{\text {old }} \cup\left\{\vec{X}_{n}\right\}_{\text {new }}\right)\right]-\left[\left\{\vec{X}_{1}\right\}_{\text {old }} \times\right.$

\footnotetext{
${ }^{2}$ In a memory-restricted environment, it is alternatively possible to perform iterations $\mathbf{7 - 1 1}$ simultaneously for the respective classes, retaining only those points of coincidence between the various class probabilities: a significantly smaller set than the matrix specified in $\mathbf{5}$. The total memory footprint for this configuration is of the order $\{X\}^{a_{1}}+\{X\}^{a_{2}}+\ldots\{X\}^{a_{n}}$, rather than the former; $\{X\}^{a_{1}+a_{2}+\ldots+a_{n}}$ (for feature-spaces of uniform dimensional size $X$ ); which is to say, an equivalent memory requirement to conventional linear methods of combination.
} 
$\left.\left\{\vec{X}_{2}\right\}_{\text {old }} \ldots\left\{\vec{X}_{n}\right\}_{\text {old }}\right]$

( $t$ the cycle count number, $N$ as above).

10. Increment the cycle counter, $t$, by 1 and go to 7 while $P_{a_{i}}>0, \forall i$.

11. After termination of the major cycle $\mathbf{7 - 1 1}$, subtract a value $t . d z$ from each point of the deconvolution matrices to establish true PDFs, if required (see footnote 5).

12. Repeat from $\mathbf{2}$ for the remaining classes in the sequence $\omega_{1}, \omega_{2} \ldots \omega_{m}$.

13. Construct the modified Bayes optimal decision boundaries at points of transition of the most probable class PDFs (cf footnote 5).

In functional mapping terms we thus seek to repeatedly perform the conditional iteration: 


$$
\begin{aligned}
& \forall t P_{t}^{\mathrm{NEW}}=\left\{\begin{array}{cc}
P_{t}^{\max }-\Delta P_{t} & \forall \vec{X}_{t}: P_{t}\left(\vec{X}_{t}\right)>P_{t}^{\max }-\Delta P_{t} \\
P_{t}\left(\vec{X}_{t}\right) & \text { otherwise }
\end{array}\right. \\
& \text { where } \Delta P_{t}=\Delta z \times\left|\vec{X}_{1}: P_{1}\left(\vec{X}_{1}\right)=P_{1}^{\max }\right| \\
& \times\left|\vec{X}_{2}: P_{2}\left(\vec{X}_{2}\right)=P_{2}^{\max }\right| \times \ldots \times\left|\vec{X}_{t-1}: P_{t-1}\left(\vec{X}_{t-1}\right)=P_{t-1}^{\max }\right| \\
& \times\left|\vec{X}_{t+1}: P_{t+1}\left(\vec{X}_{t+1}\right)=P_{t+1}^{\max }\right| \times \ldots \times\left|\vec{X}_{n}: P_{n}\left(\vec{X}_{n}\right)=P_{n}^{\max }\right| \\
& \text { while } \exists \vec{X}_{t}: P_{t}\left(\vec{X}_{t}\right)>0 \\
& P_{T O M}^{\mathrm{NEW}}\left(\vec{X}_{\mathrm{TOM}}\right)=\left\{\begin{array}{cc}
P_{\mathrm{TOM}}\left(\vec{X}_{\mathrm{TOM}}\right)+\Delta z & \forall \vec{X}_{\mathrm{TOM}}, t: P_{t}\left(\vec{X}_{\mathrm{TOM}} \cdot \vec{X}_{t}\right)> \\
P_{t}\left(\vec{X}_{\mathrm{TOM}}\right) & P_{t}^{\max }-\Delta P_{t}
\end{array}\right. \\
& \forall \vec{X}_{\mathrm{TOM}} P_{\mathrm{TOM}}\left(\vec{X}_{\mathrm{TOM}}\right)=P_{\mathrm{TOM}}^{\mathrm{NEW}}\left(\vec{X}_{\mathrm{TOM}}\right) \\
& \forall \vec{X}_{t}, t P_{t}\left(\vec{X}_{t}\right)=P_{t}^{\mathrm{NEW}}\left(\vec{X}_{t}\right)
\end{aligned}
$$

The final $P_{\text {TOM }}$ function being the tomographically reconstructed probability density function (which is initiated with uniform zero values; $\left.P_{\mathrm{TOM}}\left(\vec{X}_{\mathrm{TOM}}\right)=0 \forall \vec{X}_{\mathrm{TOM}}\right)$.

\subsection{Final Considerations on the Post-Combination Approach}

We have thus set out to significantly reduce the computation time involved in the postfiltration form of the morphologically optimal tomographic fusion strategy, and have achieved a reduction of many orders of magnitude. This is sufficient that the method no longer poses any significant cost obstacle to the implementation of the procedure with 
current computer technology (though note, this is not necessarily more efficient than performing a full classification of the composite feature space: we are assuming that under-fitting constraints prevent this possibility at the feature selection level).

The basis of this efficiency gain is the appreciation that, when viewed in terms of the constituent PDFs, the three chief computational components within the recursive procedure (the peak-seek, the analysis of the correlation between detected peaks and the subtraction/registration of those correlated components) need not be performed on an individual basis, potentially reducing the iteration requirement from $X^{n}[X]^{n}\left[X^{n-1}+X\right]$ computational cycles to $X^{n}$ computational cycles, with the further possibility of an order of magnitude decrease in this figure for point-wise continuous classifiers. This is in addition to gains arising from the requirement that the $\delta z$ parameter effectively vary throughout the procedure.

It might thus be anticipated that this PDF-centered approach could ultimately lend itself to a future reinterpretation of the morphologically optimal methodology for multiple expert fusion without any explicit reference to tomography theory, being rendering instead in the more familiar terminology of probability theory and correlation analysis.

\section{An Example Application}

Utilizing the post-filtration method thus outlined we shall, in the following section ${ }^{3}$, seek to give a practical implementation of methodology in regard to real-world data, demonstrating the sort of classification performance that we might thereby expect from a given set of classifiers under typical conditions.

\footnotetext{
${ }^{3}$ C 2003 IEEE. Reprinted, with permission, from IEEE PAMI, Vol 25, no. 3, March 2003
} 


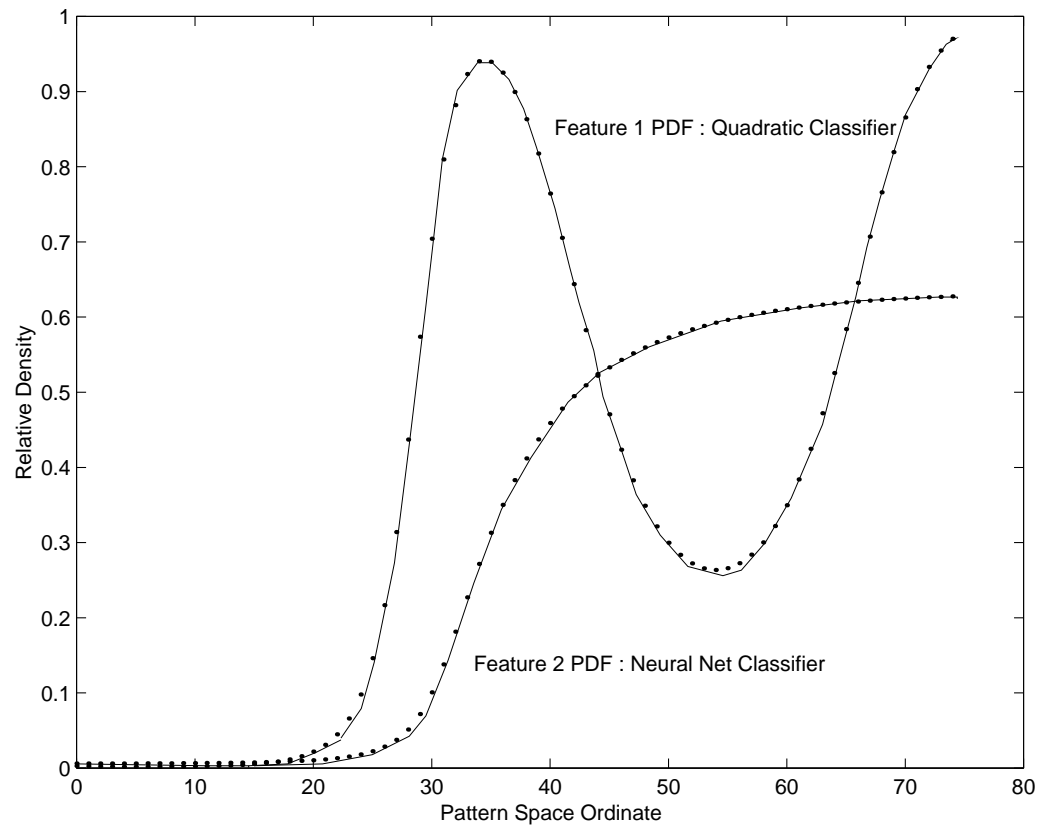

Figure 10: Class 1 feature PDFs

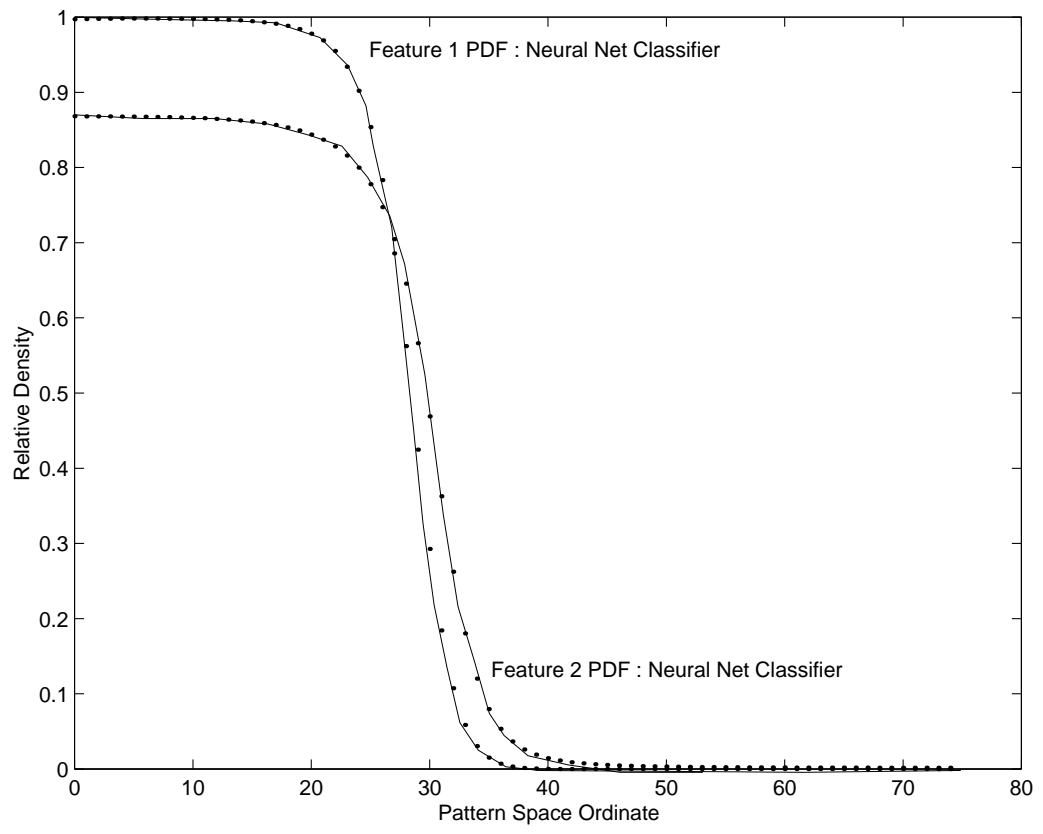

Figure 11: Class 2 feature PDFs 


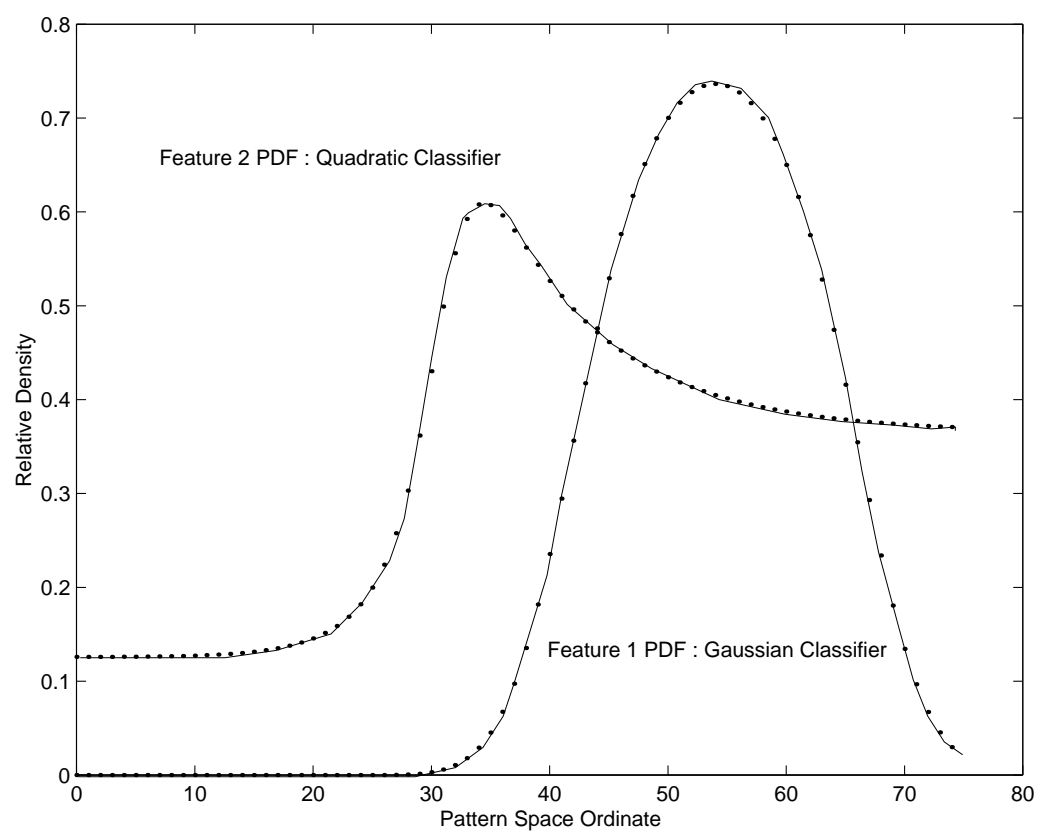

Figure 12: Class 3 feature PDFs

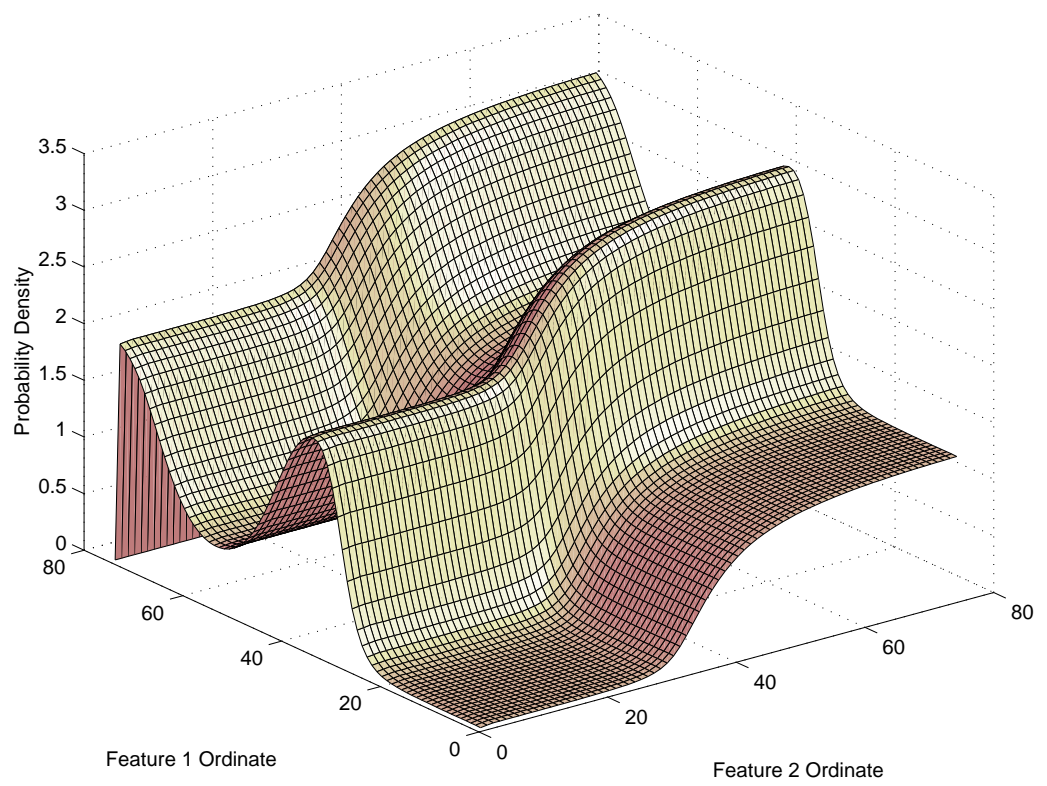

Figure 13: Class 1 2-D reconstruction via the Sum Rule 


\subsection{Test-Data Characteristics}

In setting about this goal, we have employed data consisting in a set of expertly-classified geological survey images, with subsequent image processing carried out via a battery of 26 cell-based processes for texture characterization, chosen without regard to the particular nature of the classification problem. Hence, at the outset, a particularly high feature redundancy was anticipated for the corresponding 26-dimensional pattern vector.

One such image, characterized as delineating three distinct strata classes, and exhibiting a high degree of class membership ambiguity among those classes, gave rise to a purely two-dimensional reconstructive feature space on being classified from among a bank of four potential classifiers of suitably distinct character (nearest neighbor, neural net, Gaussian and quadratic classifiers), when allocated features on a sequential forward selection basis. That is; the feature selection process involved the consecutive and independent allocation of the same two respective features to two of the four possible classifiers for each of the three classes. Thus the feature selection gave rise to a classification that explicitly excluded the combination of features within single classifiers. We shall assert that, despite being of an inherently inexhaustive sequential type (within which only two classifiers are allocated a single feature before over-classification sets in and the procedure terminates), the feature selection procedure may almost certainly be considered the equivalent of the exhaustive variety for this particular case, by virtue of the fact that any possible combination of features within one particular classifier, as selected by some putatively exhaustive feature selection algorithm, would almost invariably include the first feature allocated to that classifier on a sequential forward selection basis. Thus, the fact that the addition of any of the remaining features to this classi- 
fier within the latter regime actually degrades the performance (the method implicitly testing all of the possible feature additions), would strongly suggest that the exhaustive procedure would not find an alternative optimal solution, the over-classification effect predominating for this image.

In addition to this implicit exhaustivity of the feature selection mechanism, this particular data set lends itself to our practical investigation on the grounds that, the selected features numbering merely two, only the first stage of the n-D tomographic reconstruction algorithm need be implemented: however, and more conveniently, we have also that the resulting classifier PDF data lends itself to immediate and uncomplicated graphical representation, should any of the morphological aspects the preceding mathematical argument lack transparency, given it's necessarily abstract nature.

\subsection{Results of Application}

The three class PDFs prior to back-projection are therefore as illustrated in figures 10, 11 and 12 for the two feature axes (a normalization equalizing the extents of these axes for the purposes of display). The corresponding two-dimensional PDF reconstructions for the various classes obtained via the sum rule (that is, unfiltered inverse radon transformation) are as indicated in figures 13,14 and 15.

Filtering has the effect of rendering the reconstructed class morphology as that shown in figures 16, 17 and 18. The pronounced rectilinearity is a direct consequence of imposing the a priori precedence of the feature axes throughout the above deconvolution procedure, or, equivalently, giving an exactly equal precedence to the overlap of systematic artifacts as to artifacts themselves, in the absence of any prior assumptions as to the 


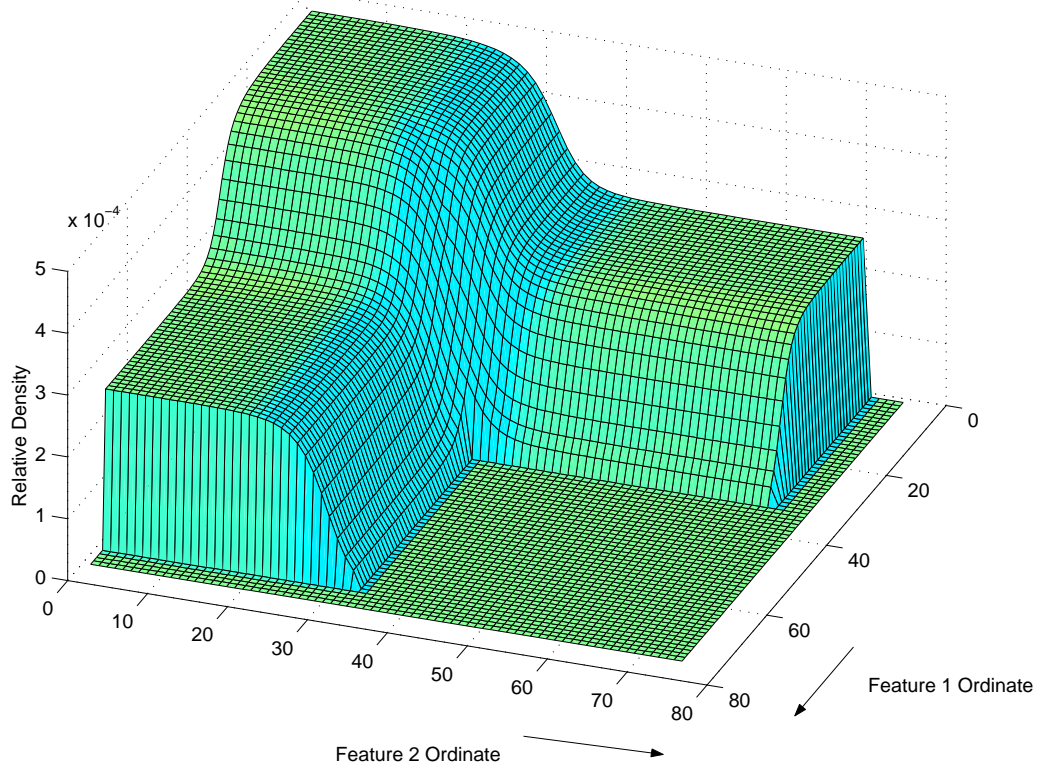

Figure 14: Class 2 2-D reconstruction via the Sum Rule

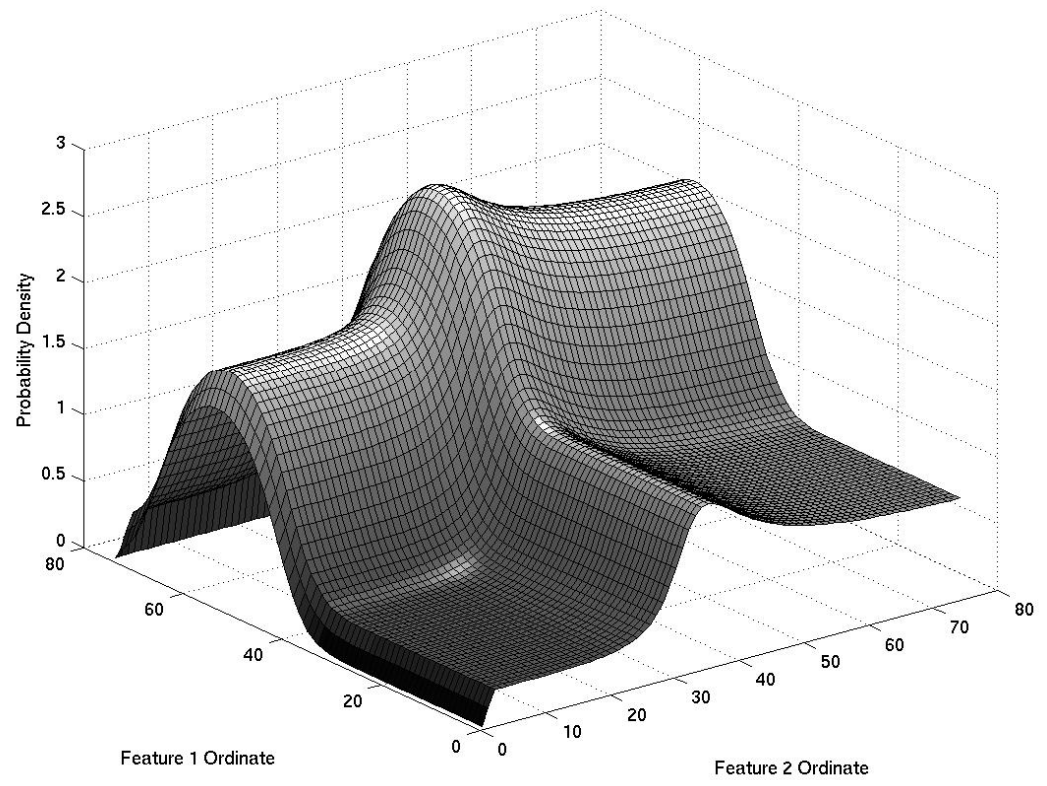

Figure 15: Class 3 2-D reconstruction via the Sum Rule 


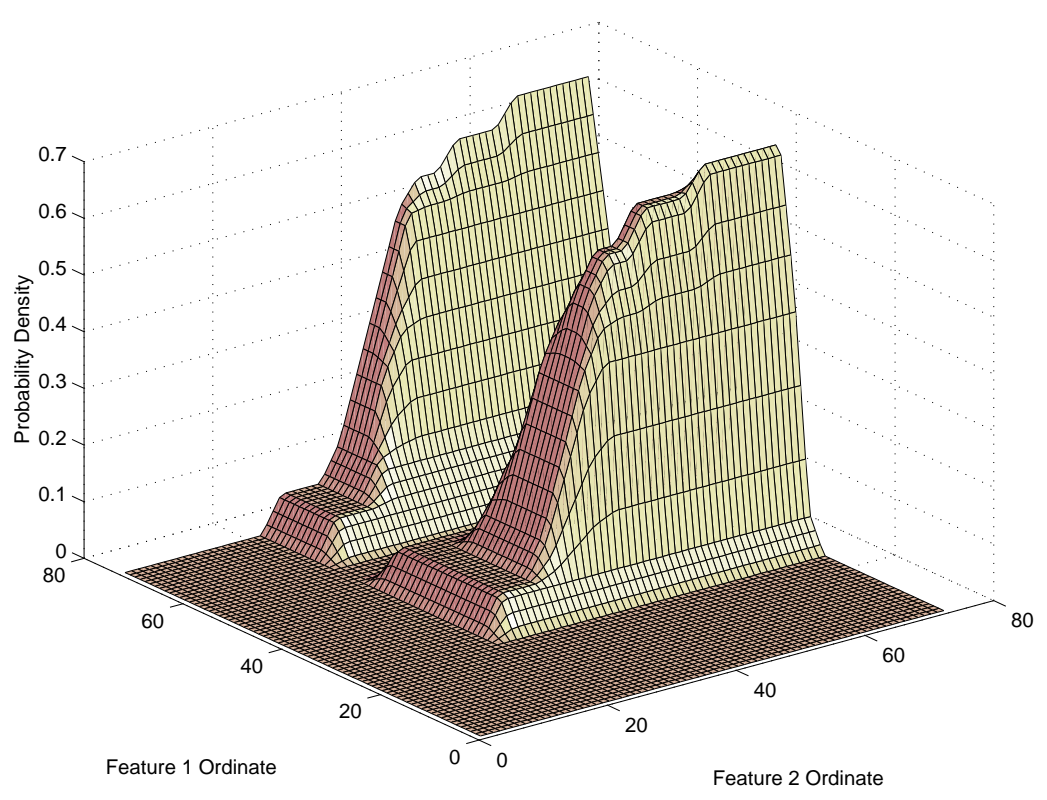

Figure 16: Class 1 2-D reconstruction after filtration

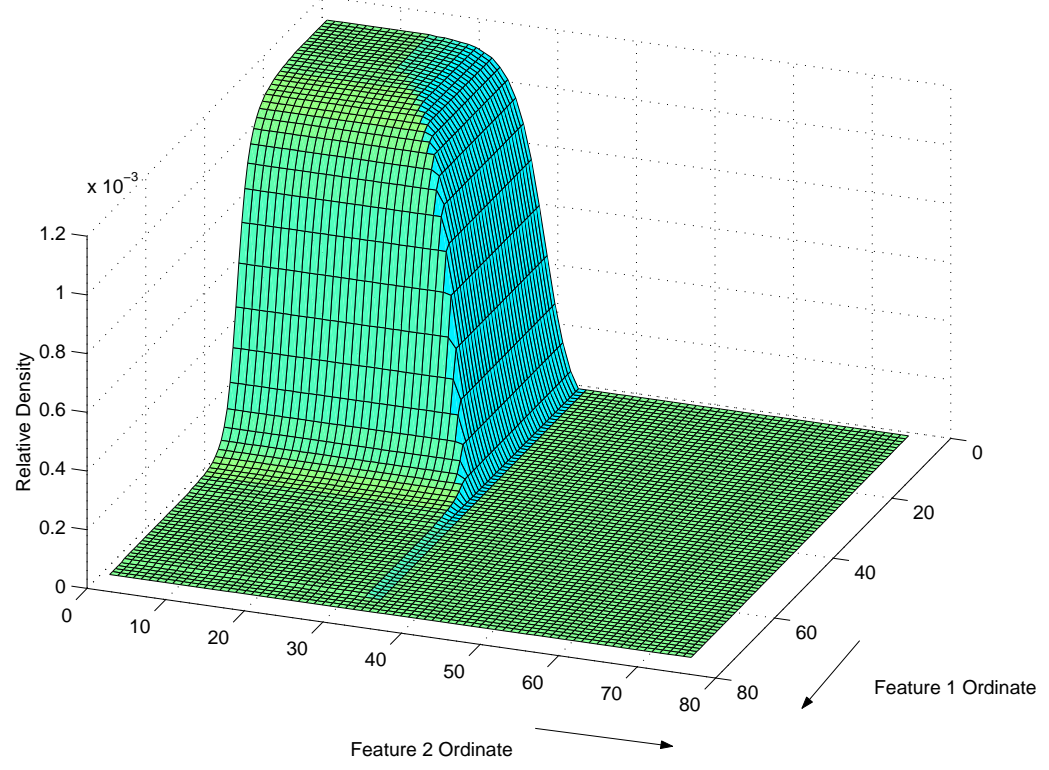

Figure 17: Class 2 2-D reconstruction after filtration 


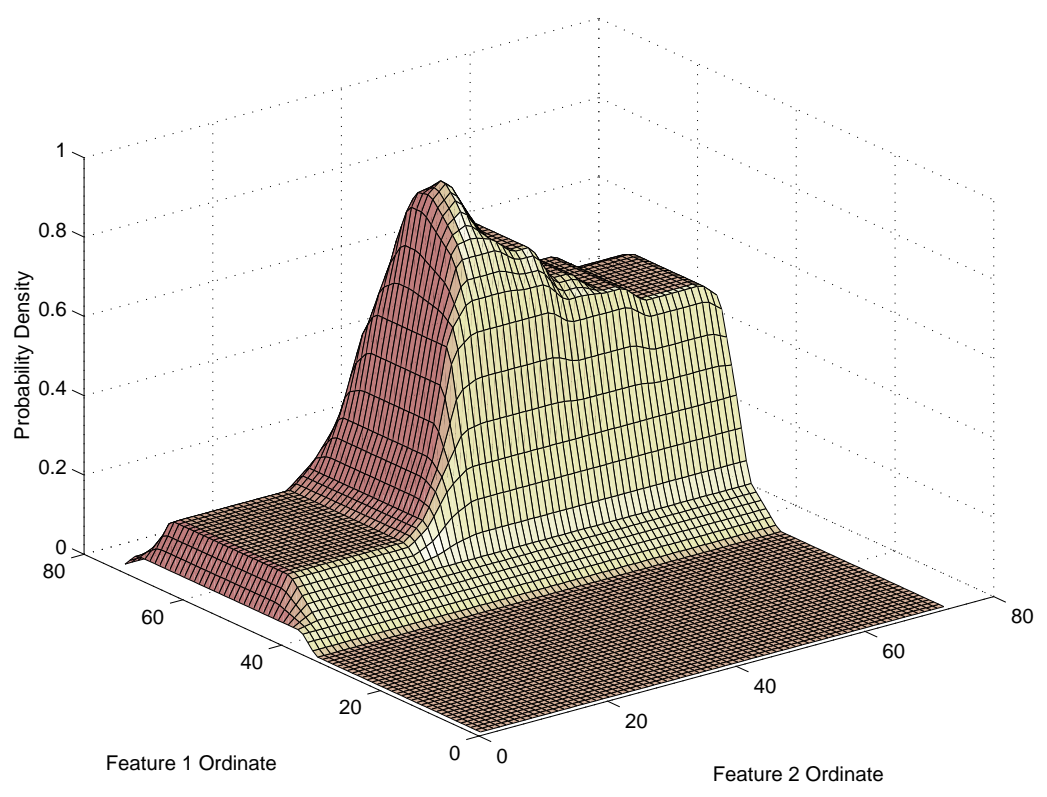

Figure 18: Class 3 2-D reconstruction after filtration

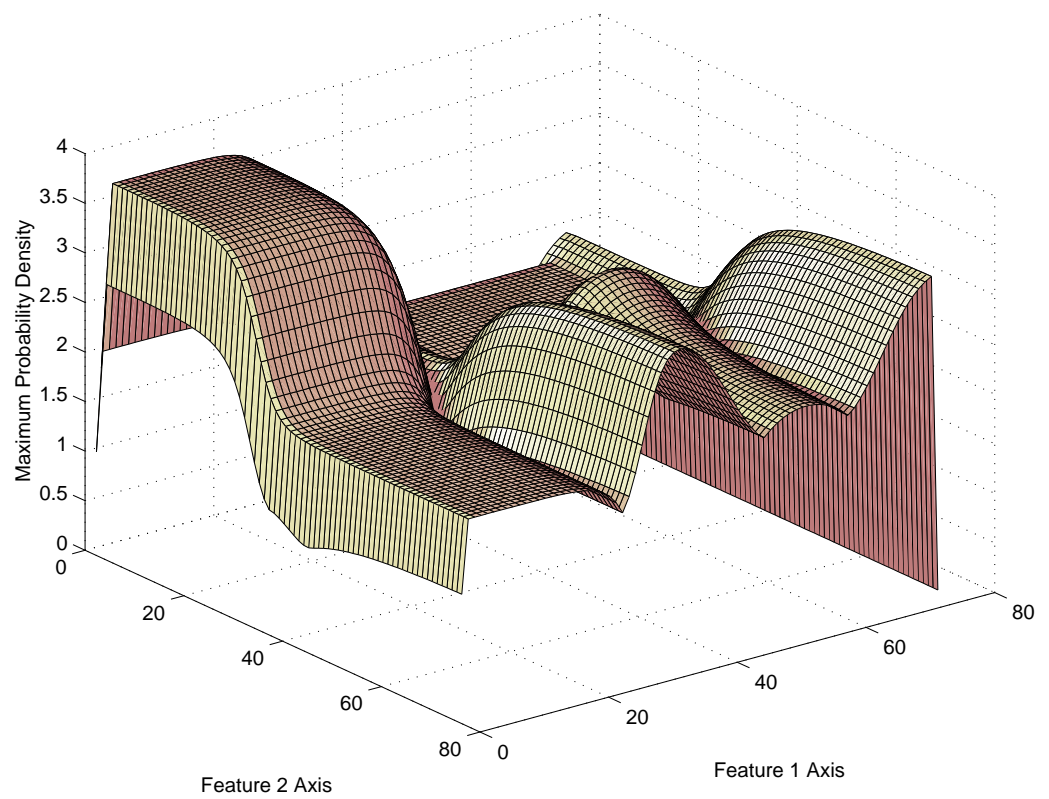

Figure 19: Composite superposition of unfiltered PDFs 


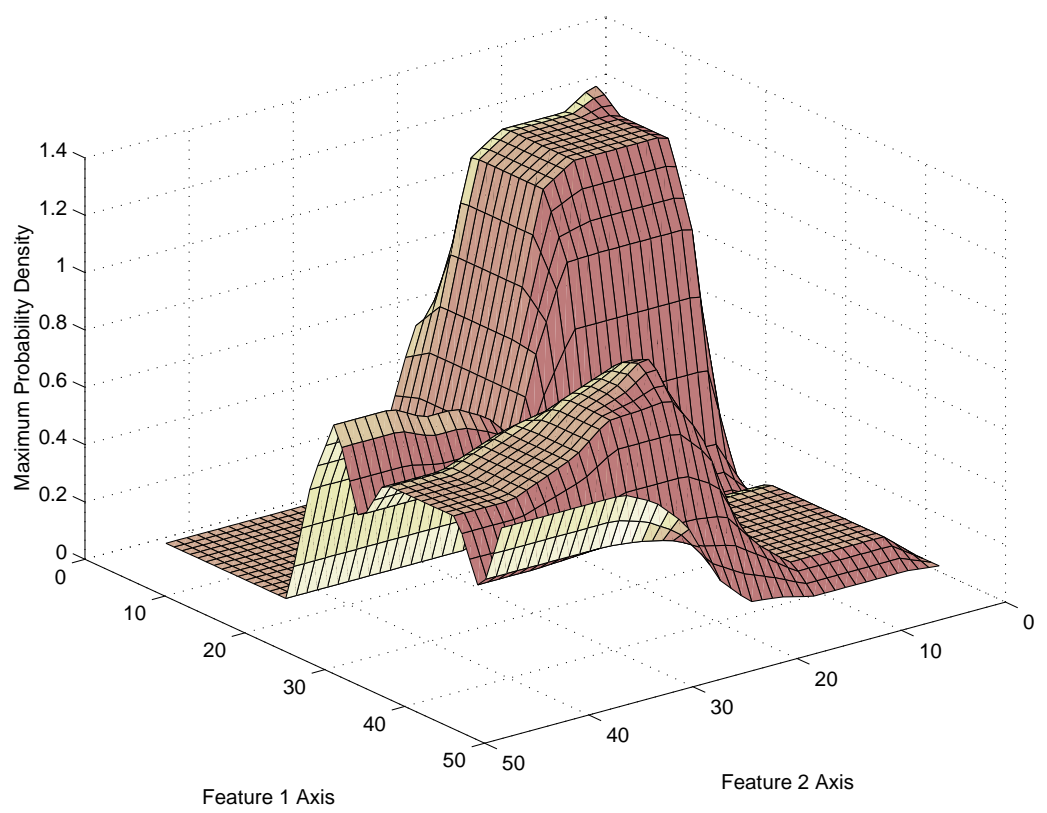

Figure 20: Composite superposition of filtered PDFs

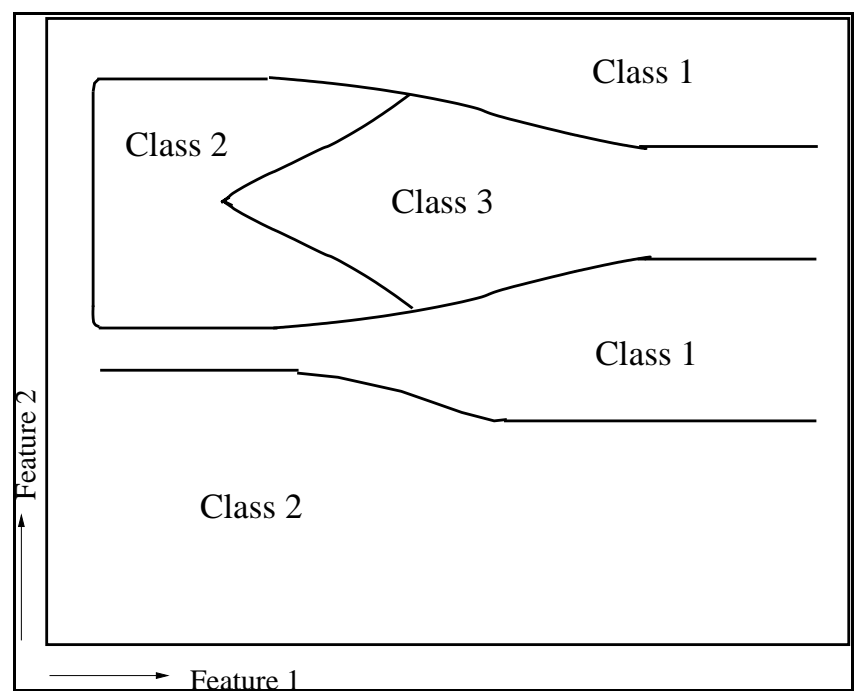

Figure 21: Decision boundaries for the unfiltered case 


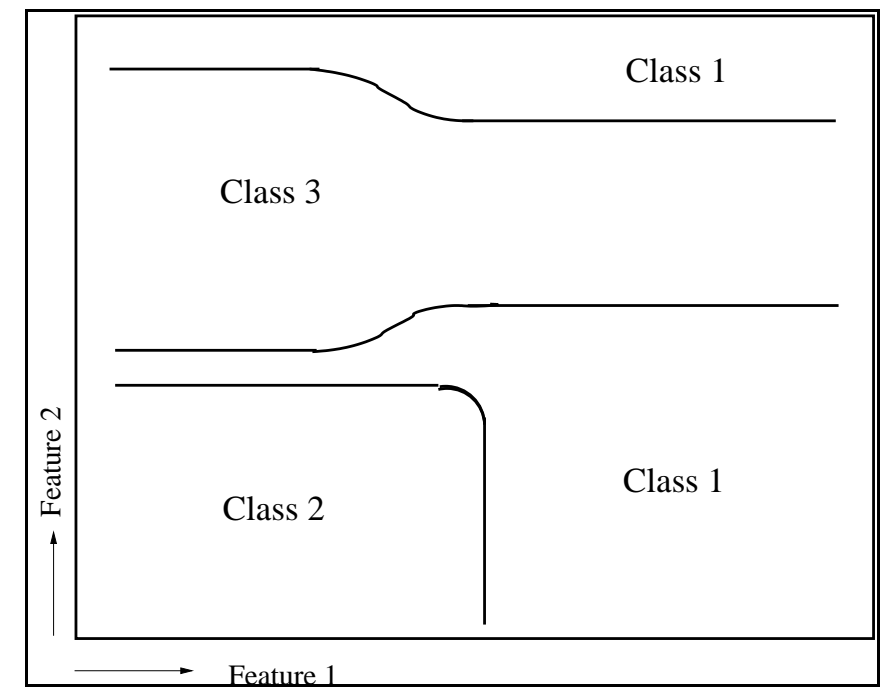

Figure 22: Decision boundaries for the filtered case

morphology of the reconstructed space. Should we wish to do so, it would of course be possible within the post-filtering approach to impose an alternative constraint on the deconvolution without having to substantially redesign the procedure, somewhat after the fashion of the maximum entropy deconvolution, which in fact requires the imposition of such a priori information [16]).

The composite superpositions of the three class PDFs over the reconstructed space, such that only the maximum of the respective probability densities is indicated, are as shown in figure 20 for the filtered, and figure 19 for the unfiltered case. However, probably the more indicative rendering of the distinction between the two approaches to classifier combination is in terms of the decision boundaries of the respective reconstructed spaces: these are given in figure 22 for the filtered space and figure 21 for the unfiltered space. It is immediately evident that the cross-like extensions along the feature axes associated with clusterings of higher probability densities are no longer evident in the filtered space. For this particular case, the most dramatic changes to the morphology of the decision space occur at some distance from the class probability maxima, and thus it is only the 
outlying pattern vectors that tend to be reclassified under the filtered regime, which hence represents only a relatively minor percentile change in the overall classification rate (the probability of miss-classification, though, undergoes a far more substantial percentage change). In the more general scenario, however, it is entirely possible that a substantial fraction of a class's extent within the unfiltered reconstructed probability space may in fact be occluded by the sampling geometry of another class's reconstructed PDF, in which case a very substantial percentile change in the overall classification rate would be expected.

Within our particular example, however, the probabilities of misclassification for twodimensional PDFs constructed from 1,000 of the 10,000 possible samples for the filtered and unfiltered reconstructed spaces, respectively, are then; 0.0472 and 0.0709: an approximate halving of the misclassification rate.

\section{Dimensionality Issues: Empirical and Theoretical}

\section{Constraints on the Relative Performance of To-}

\section{mographic Classifier Fusion.}

With the preceding example thus defining an approximate expectation of the level of performance improvement due to tomographic filtration, we should now like to turn to a more systematic quantification of both the classification performance and estimation error robustness of the tomographic classifier fusion methodology ${ }^{4}$.

In particular, we seek to confirm that the tomographic methodology represents a gen-

\footnotetext{
${ }^{4}$ This section (C)World Scientific (to appear in the International Journal of Pattern Recognition and Artificial Intelligence).
} 
erally optimal strategy across the entire range of problem dimensionalities, and at a sufficient margin to justify the general advocation of its use.

In section 2 it was stated without proof that the expected error resilience of the tomographic method ought to be similar to that of the sum rule (the optimal combination strategy in terms of robustness to estimation error [2]), since the back-projection aspect of the tomographic fusion approach imposes exactly the same averaging process with respect to stochastic variation. A precise calculation was omitted since it depends critically on the interaction between the filtering mechanism and the morphological characteristics of the classifier (which is not something we would wish to specify in advance, the tomographic method being intended as a 'black box' approach, to which novel methods of classification may be appended as developed). Given this theoretical limitation on characterizing the error resilience of the proposed method, it is necessary to base any attempted quantification of the resilience to estimation error instead on practical trials and model solutions in order to build a convincing case.

More generally, though, we have yet to fully establish the most significant performance statistic for the tomographic combination method in relation to the conventional alternatives: the effect on the misclassification rate. The very limited example of this statistic given in the preceding section indicates a halving of the misclassification rate. However, momentary consideration would indicate that it is not possible to guarantee an equivalent performance response for combinations of higher dimensionality without a great deal of further analysis. Indeed, this is self-evidently not the case if the classifiers constituting the combination exhibit any degree of estimation error, since error resilience scales differently with dimensionality for the sum and product rule combination schemes (see discussion in [2]). It is therefore necessary, in any reasonable attempt to quantify the 
general performance of tomographic combination, to establish performance across the range of feature-space dimensionalities: we should in particular like an assurance that the tomographic method remains the optimal choice at higher dimensionalities within a representative range of scenarios. Sections 5.1 and 5.2 shall therefore, respectively, detail our attempts to achieve this at the practical and theoretical levels.

Giving any comparative performance benchmark for the tomographic combination method requires that we test it against a representative sample of the remaining combination schemes. Kittler et al. [2] have demonstrated that the majority of commonly used decision rules can be considered to derive from either the sum or the product decision rules: It is therefore these two methodologies, in particular, against which we shall choose to benchmark the tomographic combination system outlined in section 3.4.3.

\subsection{Relative Performance of Tomographic Classifier Fusion in Empirical Tests}

For the practical, as opposed to the mathematical aspect of this investigation, the 'realworld' data upon which we shall perform this experimental comparison derives from the same set of expertly-classified geological survey images utilized earlier, ie with the 26 dimensions of the pattern vectors corresponding to 26 distinct cell-based processes for texture characterization. The arbitrary nature of these processes means that the totality of the data-set simultaneously exhibits all three of the distinct characteristics of, respectively; large-scale feature redundancy, feature independence and its converse, feature dependency within its various feature subsets: that is, very largely the full range of possible behaviors with regard to feature selection, classification and classifier com- 
bination.

Also, since we are primarily interested in testing the relative capabilities of the combination schemes, we shall seek both to homogenize the classifiers constituting the combination, and to make them as representative of the pattern-data as possible. Thus, rather than the customary arrangement in which feature sets are allocated to a morphologically disparate set of classifiers on the basis of their individual representative strengths, we shall instead artificially impose a uniform classification scheme, a probability density function derived by regularly-spaced block-density histogramming of the pattern-data, upon each of the tested feature subsets constituting the combination.

Furthermore, in order that we might establish a direct measure of the classification performance of the various combination schemes, we shall impose the condition that the composite feature space PDF of $i$-dimensions which we are implicitly reconstructing by classifier combination is that obtained by a block density histogramming of the original $i$-dimensional space. In other words, we are designating the $i$-dimensional PDF thus derived as the underlying prior probability density function of the $i$-dimensional space.

For this approach to have general validity it is necessary that a large number of pattern vectors are sampled per histogram, even at the extremity of the tested dimensionality range. Thus we are also required to impose a relatively small number of bins per feature $(r)$ in order to maintain reasonable count statistics at the extremity of the range: of the order of $r=4$, given our $\approx 125000$ pattern vectors and 8 dimensional range.

Because of the need to establish a meaningful performance comparison across the dimensional range, it is additionally necessary to derive each of the tested multi-dimensional composite reference feature-space PDFs from the same experimental source. Hence we obtain the various $i$-dimensional spaces via projection of the complete $n$-dimensional 

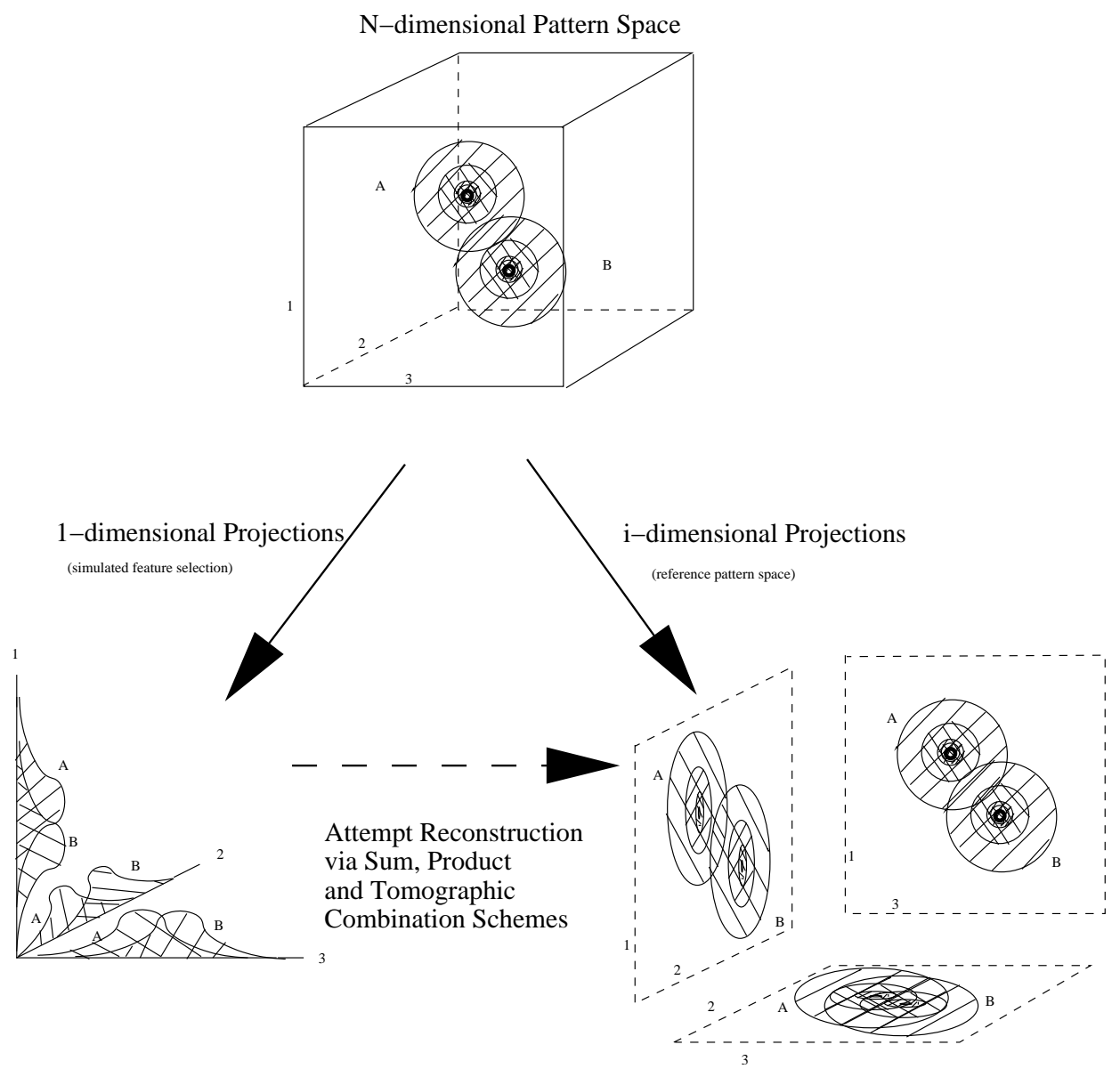

Figure 23: Experimental Format

pattern-space, finally averaging over all ${ }^{n} C_{i}$ performance figures thus obtained. Clearly, as the dimensionality $i$ varies, the averages thus obtained are subject to a statistical fluctuation associated with low number statistics (becoming asymptotic at $i=n$ when only one subspace exists), and hence the tested sequence is required to terminate well short of this value (coupled with the aforementioned consideration of avoiding under-sampling of the prior PDF at higher dimensionalities).

The reason it shall only prove necessary to consider the combination configuration consisting of $i$ one-dimensional classifiers (that is, combinations with one feature per classifier), is that we are principally interested in characterizing the variation of combination performance in relation to a uniform 'morphological information shortfall'. That is, we 
are primarily interested in the extent to which a combination scheme can make use of the $r i$ possible classifier ordinate values to reconstruct the $r^{i}$ possible $\underline{c o}$-ordinates of the prior PDF: introducing additional combinations of classifiers containing differing numbers of features would tend only to obscure this perspective without generating any additional insight into the combination processes not already encompassed by the latter approach. The experimental format for the real-world combination test is therefore as illustrated in figure 23 .

We should clarify that the test scenario in no way intended to represent a plausible real-world situation when feature-selection is explicitly taken into consideration: Given that we are in a position to obtain sufficient pattern-vectors as to be able to constrain a plausible model of the $i$-dimensional prior PDF, the most effective feature selection strategy (presuming a reasonably flexible set of classifier morphologies to choose from) would, most naturally, be to allocate the maximal $i$ features to the best performing classifier of the ensemble in order to guarantee retention of the maximal quantity of discrimination information. We have, however, imposed the one-feature-per-classifier limitation in order that we might mimic the generalized situation in which any oneclassifier parameterization of the whole $i$-dimensional space would likely be subject to serious over-parameterization error, and therefore disposed to reduce the classification rate in relation to a combination of classifiers of lower, but better sampled, feature dimensionalities. Of course, this condition being an external restriction means that, in fact, we do have access to a plausible model for the $i$-dimensional prior PDF as required for the purposes of performance evaluation.

The specified experimental scenario should thus be considered from the context of the broader tomographic perspective, within which feature-selection can be envisaged as 
seeking an appropriate balance between the mutually exclusive requirements of maximizing the retention of class-discriminant morphology information through the allocation of spaces of higher feature dimensionalities to the classifiers, and the minimization of the dangers of over-classification through the allocation of lower feature-space dimensionalities to classifiers.

\subsubsection{Response to Estimation Error}

The remaining aspect of the investigation, the assessment of the resilience to estimation error of the three fusion methods, is addressed in the above experimental context by the straightforward simulation of classifier error through adding uniform stochastic noise to each of the classifier density histograms (simulating, in effect, estimation-error arising from an insufficient degree of parameter-freedom among the classifiers, rather than estimation-error attributable to, say, incorrect, or over, parameterization).

The tomographic performance results for the 'real-world' geological survey data are thus as depicted in figure 27 of section 5.6, alongside an analysis of its comparative significance. Placing the experiment in the widest context, however, requires that we turn to a more constrained model scenario:

\subsection{Relative Performance of Tomographic Classifier Fusion on Model Data}

The significance of the findings of the preceding investigation are, then, best established in relation to an absolute baseline against which the performance on real-world data may be graded. Any such proposed performance indicator must thus seek to determine 
the effect of classifier combination on the classification performance in a way that is independent of both pattern-data, as well as, classifier morphology.

It so transpires that one of the very few classes of mathematically tractable characterizations of the algorithmic procedure of section 3.4.3 occurs in relation to prior PDFs composed of orthogonally-gridded histograms of randomized density (hence fulfilling the requisite test conditions of independence to pattern-data morphology in the case where every such distribution is considered). Furthermore, it is natural to suppose that prior probability density distributions so derived will, when averaged over the ensemble, naturally constitute a generalized performance minimum for the tomographic methodology as a consequence of its specifically seeking to reconstruct the overall pattern-space PDF through correlating morphology across the separate classifiers: a randomized morphology effectively undermines this agenda by decorrelating the differing subregions of the composite PDF, permitting the isolation of the required 'absolute' performance statistic. $^{5}$

In combination with this argument, there is also the consideration that the randomization of the PDF morphology takes places with respect to a coordinatization aligned with the feature axes: tomographic methods, however, in applying a prior filtration to the back-projected radon data, implicitly seek to override metrics dictated by the feature axes in favor of those constrained solely by the underlying classifier morphology. There are therefore two distinct sets of reasons for supposing that the specified ensemble of prior PDF forms constitutes a generalized performance minimum with regard to the tomographic combination method, which (when combined with their unique mathe-

\footnotetext{
${ }^{5} \mathrm{~A}$ performance maximum for the tomographic method is correspondingly established when the composite prior PDFs correspond to unimodal distributions that are capable of undergoing decomposition into intersecting hypercubes, in which case the tomographic combination performance achieves Bayes optimality (on the assumption of ideal constituent classifiers).
} 
matical tractability), naturally cement them as the choice for mathematical analysis of the relative tomographic combination performance: the derivation of this quantity will therefore occupy the majority of the remainder of the section.

An additional benefit arising from the elucidation of the model data performance statistics for all three of the tested combination methods is that, in doing so, we uncover a great many of the mathematical processes that underly the performance/dimensionality scaling phenomenon for classifier combiners in general. Remarkably, however, we shall demonstrate that the tomographic fusion method, notwithstanding the specified PDF restrictions, still exceeds the performance of the sum and product rules by a considerable margin.

\subsection{Tomographic Model Solution}

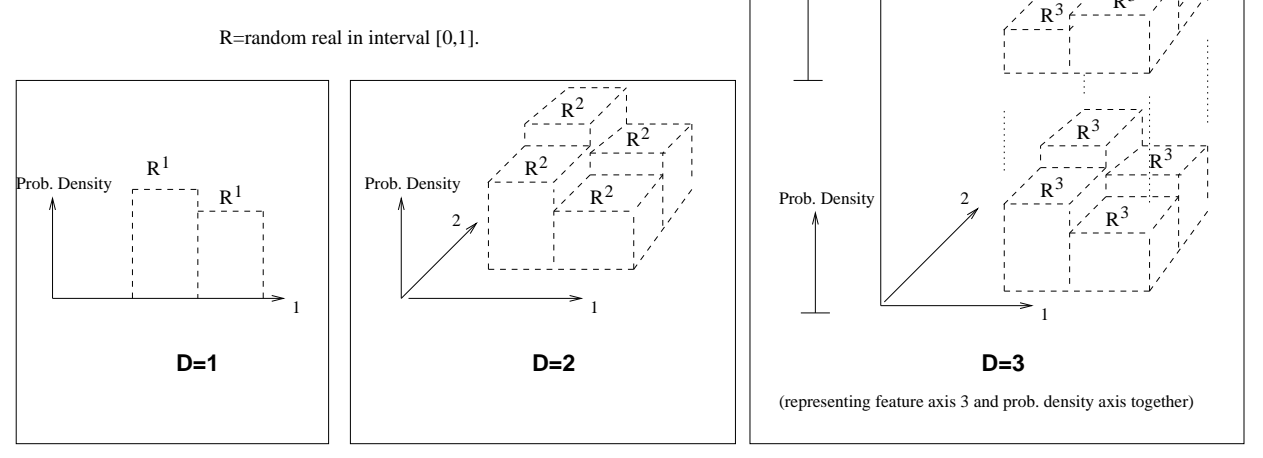

Figure 24: Randomized Composite PDF Morphology of Dimensionality $i=1,2,3$

The composite prior PDF format for the model solution is then as illustrated in figure 24 for the first three dimensionalities in the range, with the obvious extrapolation to higher dimensionalities. The per-ordinal resolution, $r$, of the gridded composite PDF having thus been lowered to a value of 2 , it becomes possible to uniquely grade the ordinal 
projections in the manner represented in figure 25 , the ordinal disparity thus, now, the single distinguishing parameter between classifier morphologies. That this reformatting is permissible within the context of the model data performance test is a consequence of the tomographic combination methodology's situation-specific independence to ordinate translations when the indicated prior PDF constraints are imposed, along with its more generalized independence to axial permutations occurring irrespective of any PDF model constraint. It shall therefore be the case, throughout the paper, that we continue the convention already adopted in figure 25; namely, that calligraphic figures (such as $\mathcal{A}$ ) denote such magnitude-ordering of ordinal density values.

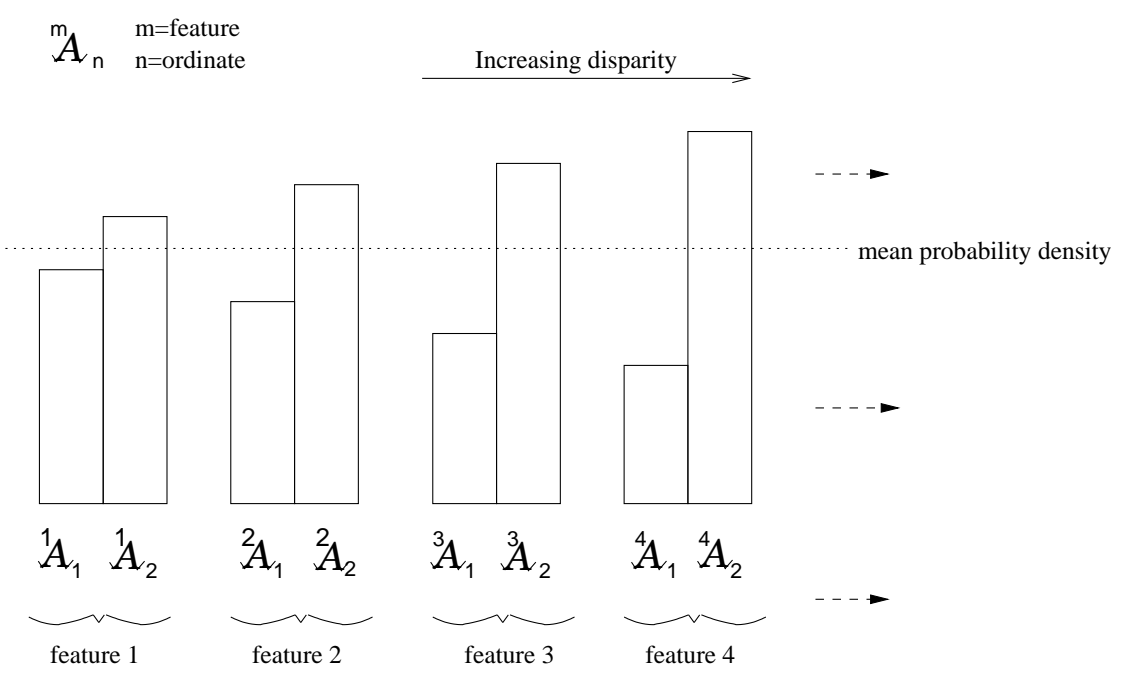

Figure 25: Projected Ordinal PDF Values for the Various 1-D Classifiers

The process outlined in section 3.4.3 may now be seen as a sequence of subtractions bringing the respective classifier ordinate values, ${ }^{x} \mathcal{A}_{2}$, into equality with the values of their neighboring ordinates, ${ }^{x-1} \mathcal{A}_{2}$, the number and magnitude of the subtractions enacted with respect to each set of $i$-dimensional ordinates thus dictating the tomo-

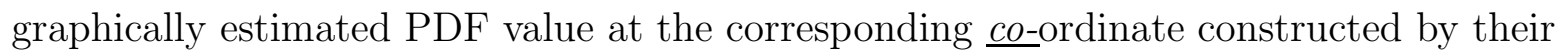
amalgamation. Since each subtraction removes a constant quantity, $\propto$ ( probability 
density $\times$ number of ordinates), from each $\mathrm{PDF}$, the corresponding per co-ordinate increment in the proposed composite PDF at each iteration increases in the geometric sequence $2^{1}, 2^{2}, 2^{3} \ldots$ Hence the actual value of the composite PDF proposed by tomographic fusion at a particular $i$-dimensional coordinate is thus:

$$
\left.P_{\text {tom. }}(\vec{a})={ }^{1} \mathcal{A}_{i}\left(\frac{1}{2}\right)^{i-1}+\sum_{m=1}^{\bar{a}}\left({ }^{2} \mathcal{A}_{i-m+1}-{ }^{1} \mathcal{A}_{i-m+1}-{ }^{2} \mathcal{A}_{i-m}+{ }^{1} \mathcal{A}_{i-m}\right)\left(\frac{1}{2}\right)^{i-m}\right)
$$

where $\vec{a}$ is the coordinate $\left(a_{1}, a_{2}, a_{3}, a_{4} . . a_{i}\right)\left(a_{x}\right.$ may take the values 1 or 2 denoting the minimum and maximum ordinate values, respectively). The term $\bar{a}$ refers to the minimum $x$ value for which $a_{x}=1$. (We also specify that ${ }^{x} \mathcal{A}_{0}=0$ for consistency).

Thus we see that the predicted PDF value is governed by a cumulation of the disparities between ordinal projections, rather than simply those particular ordinates intersecting the point under consideration, as is the case for the sum rule and product rules (and indeed any other linear combination technique). In this way, even under the simplified scenario dealt with here, the tomographic technique involves all of the information contained within the ordinal projections (as the classifier PDFs) to generate the predicted prior probability density value.

The particular quantity that will be of interest to us in establishing the classification performance of the tomographic combination method is the PDF of the predicted composite probability density value at a particular $i$-dimensional coordinate in relation to a given composite probability value. That is, since we are seeking to establish a morphologyindependent classifier combination performance estimate, we shall derive a predicted composite feature-space PDF value distribution function from the ensemble of all pos- 
sible prior composite PDF morphologies within the terms set out above.

The first step in this process is to establish the ensemble average PDFs of the individual classifiers' projected ordinate values, $\left({ }^{x} A_{1,2}\right)$, in relation to a particular fixed prior PDF value, $X$, occurring at the coordinate $\left(a_{1}=1, a_{2}=1, a_{3}=1 \ldots a_{i}=1\right)$ (that is, fixed relative to the ensemble averaging over all prior composite PDFs consistent with this condition). Note that we are now in the original coordinate system, so the superscript numeral has no bearing on the relative value of $A$. The value of the prior density function at each $i$-dimensional coordinate (excluding $\left(a_{1}=1, a_{2}=1, a_{3}=1 \ldots a_{i}=1\right)$ ) is thus permitted to take, independently, a uniformly distributed random value in the interval $[0,1]$. (We need not consider the issue of normalization at this stage).

Once this quantity has been established, the resulting formulation will then permit a calculation of the disparity values, the predicted composite PDF value being constructed, as indicated, by a series of iterations whose total number is governed by the index number of first positive disparity value (of the $i$ total): that is, the first pair of feature ordinate values, ${ }^{x} A_{1,2}$, for which the unconstrained ordinate value ${ }^{x} A_{2}$ is greater than the constrained one; ${ }^{x} A_{1}$.

The degree to which the probability distribution of ordinal disparities is constrained by the actual value of the point under consideration depends, primarily, upon the dimensionality $i$ of the problem, a point we can elucidate by commencing with the calculation of the distribution of those ordinate projection values, ${ }^{x} A_{2}$, that do not intersect the point under consideration (and are therefore not in anyway constrained by it, given the randomness inherent in the PDF specification). This quantity is derived via convolution of the PDFs of the independent histogram density parameters comprising the composite feature-space PDF, ${ }^{x} A_{2}$ being essentially a sum over independent random variates: 


$$
P\left({ }^{x} A_{2}\right) d^{x} A_{2}=\overbrace{\Pi \star \Pi \star \ldots \sqcap}^{2^{i-1} \text { convolutions }} d A^{\prime} \equiv(\sqcap \star)^{2^{i-1}}
$$

(with the later term adopted as a convention throughout: $\sqcap$ is the probability density of the uniformly distributed random variate with limits $[0,1])$

That is, the distribution of ${ }^{x} A_{2}$ approaches the Gaussian form in the limit $i=\infty$ via the central limit theorem. Equation 70 may be written without explicit convolution as:

$$
P_{i}\left({ }^{x} A_{2}\right)=\frac{1}{2(i-1) !} \sum_{k=0}^{i}(-1)^{k}\left(\begin{array}{l}
i \\
k
\end{array}\right)\left({ }^{x} A_{2}-k\right)^{i-1} \operatorname{sgn}\left({ }^{x} A_{2}-k\right),
$$

via the characteristic function method.

Conversely, those ordinate projection values that do intersect the point under consideration (being therefore partially constrained by it), ${ }^{m} A_{1}$, are distributed thus:

$$
P\left({ }^{m} A_{1}-\vec{X}\right) d^{m} A_{1}=\overbrace{\Pi \star \Pi \star \ldots \sqcap}^{2^{i-1}-1 \text { convolutions }} d^{m} A_{1} \equiv(\sqcap \star)^{2^{i-1}-1}
$$

where the constraining factor that the point $\left(a_{1}=1, a_{2}=1, a_{3}=1 \ldots a_{i}=1\right)$ must equate to the value $X$ acts to displace the distribution (minus one of the convolutions) by that same value (a point that may be readily confirmed by setting one of the $\sqcap$ in equation 70 to a delta function centered on $X$ ).

The probability that any given feature, $j$, has a disparity $D_{j}={ }^{j} A_{2}-{ }^{j} A_{1}$ between ordinate projections is hence:

$$
P\left(D_{j} \mid X\right) d D_{j}=\int_{0}^{D}(\sqcap \star)^{2^{i-1}-1}\left({ }^{j} A_{1}-X\right)(\sqcap \star)^{2^{i-1}}\left({ }^{j} A_{1}+D_{j}\right) d\left({ }^{j} A_{1}\right) d D_{j}
$$


(with a negative value indicating ${ }^{j} A_{2}>^{j} A_{1}$ )

Recognizing the above as essentially a convolution with one of the functions having undergone the ordinate inversion $(A \rightarrow-A)$, giving a total of $2^{i-1}$ convolutions of the uniform distribution, we may re-write equation 73 as:

$$
P\left(D_{j} \mid X\right) d D_{j}=(\sqcap \star)^{2^{i-1}}\left(D-X-2^{i-1}\right) d D
$$

(the $-2^{i-1}$ term recentering the distribution to account for the [now implicit] ordinate inversion)

Critically, whilst the individual sets of $A$ values for each of the ordinal projections are not independent of each other, their differentials $D$ are (perturbations of any particular $D$ value affect the ${ }^{x} A_{2}$ and ${ }^{x} A_{1}$ values of the other ordinates symmetrically). Hence, we can derive the probability distribution of the predicted tomographic combination rule $X$ values, $\left(P_{\text {tom. }}\right)$, by considering the various $D_{j}$ values independently, and making the appropriate $D$ for $A$ substitutions in equation 70 . This comes about in the following way:

In the original coordinate system, the value $\bar{a}$ (the minimum $x$ value for which $a_{x}=1$ ) becomes instead $i$ minus the largest $x$ for which the corresponding $D_{x}$ value is less than zero (that is, for which ${ }^{1} A_{x}>^{2} A_{x}$ ), and the summation is hence over those $D$ magnitudes that are greater than $\left|D_{x}\right|$.

The revised format of equation 70 is therefore: 
$P_{\text {tom. }}\left(a_{1}, a_{2}, \ldots a_{i}\right)=A_{m i n}\left(\frac{1}{2}\right)^{i-1}+\left|D_{i}\right|\left(\frac{1}{2}\right)^{i-1}-\left|D_{i-\bar{a}}\right|\left(\frac{1}{2}\right)^{i-\bar{a}}+\sum_{m=1}^{\bar{a}-1}\left|D_{i-m}\right|\left(\frac{1}{2}\right)^{i-m}$

(for $\bar{a}>2$, otherwise we would be required to remove the second and third terms as appropriate)

Thus, for a given $\bar{a}$ the probability of a particular predicted prior PDF value, $P_{\text {tom. }}$ occurring at the point $(1,1, \ldots)$ with respect to an actual underlying value, $X$, is:

$$
P\left(P_{\text {tom. }} \mid \bar{a}, X\right)=
$$

$P\left(A_{\min } 2^{i-1}\right) \star P\left(\left|D_{i}\right| 2^{i-1}\right) \star P\left(-\left|D_{i-\bar{a}}\right| 2^{i-\bar{a}}\right) \star P\left(\left|D_{i-1}\right| 2^{i-1}\right) \star P\left(\left|D_{i-2}\right| 2^{i-2}\right) \star \ldots \star P\left(\left|D_{i-\bar{a}+1}\right| 2^{i-\bar{a}+1}\right)(76)$

The probability that a particular $\mathrm{D}$ value is the most positive of the negative $D$ values (ie that $D=D_{\bar{a}}$ ) is given by:

$$
\begin{aligned}
P\left(D=D_{\bar{a}} \mid \bar{a}\right) & ={ }^{i} C_{1}\left[P\left(D>D_{\bar{a}} \& D<0\right)\right]\left[1-P\left(D>D_{\bar{a}} \& D<0\right)\right]^{i-1} \\
& ={ }^{i} C_{1}\left[\int_{0}^{D_{\bar{a}}} P(D) d D\right]\left[1-\int_{0}^{D_{\bar{a}}} P(D) d D\right]^{i-1}
\end{aligned}
$$

(the probability with which the number of terms, $\bar{a}$, is distributed being:)

$$
\begin{aligned}
P\left(\bar{a} \mid D_{\bar{a}}\right) & =\int_{0}^{-\infty}{ }^{i} C_{\bar{a}}\left[P\left(D<-D_{\bar{a}} \& D>0\right)\right]^{\bar{a}}\left[1-P\left(D<-D_{\bar{a}} \& D>0\right)\right]^{i-\bar{a}} P(D \gamma(a \Phi) \\
& =\int_{0}^{-\infty}{ }^{i} C_{\bar{a}}\left[\int_{0}^{-D_{\bar{a}}} P(D) d D\right]^{\bar{a}}\left[1-\int_{0}^{-D_{\bar{a}}} P(D) d D\right]^{i-\bar{a}} P(D) d D
\end{aligned}
$$


Given, then, that there are $\bar{a}$ terms, the probability distribution of those $D$ terms that do form the summation (that is the $D_{k}$ such that $1<k<\bar{a}$ ) is:

$$
P\left(D_{k} \mid D_{\bar{a}}, \bar{a}\right)={ }^{\bar{a}} C_{k}\left[P\left(D<D_{k}\right)\right]^{k-1} P\left(D=D_{k}\right)\left[P\left(D>D_{k} \& D<D_{\bar{a}}\right)\right]^{\bar{a}-1}[P(D>0)]^{i-\bar{a}}
$$

Thus, substituting the above in to equation 76 , and eliminating $\bar{a}$ by summing over every possibility, we eventually obtain the sought quantity, $P_{\text {tom. }}$; the probability distribution of the predicted composite probability density value at $(1,1,1 \ldots)$ under tomographic combination with respect to the true value $X$ :

$$
P\left(P_{\text {tom. }} \mid X\right)=\sum_{\bar{a}=1}^{i} \prod_{k=1}^{k=\bar{a}} P\left(D_{k} \mid D_{\bar{a}}, \bar{a}\right) \int_{-\infty}^{-\infty} P\left(\bar{a} \mid D_{\bar{a}}\right) P\left(D_{\bar{a}}\right) d D_{\bar{a}} P\left(P_{\text {tom. }} \mid \bar{a}, X\right)
$$

(the outstanding term, $A_{\text {min }}$, in the above being eliminated by summing over the two possibilities $A_{\min }={ }^{x} A_{1}, A_{\min }={ }^{x} A_{2}$ ). For our purposes, it will be sufficient to carry out this integration numerically.

Given that the tomographic method makes optimal use of the morphological information contained in the classifiers constituting the combination, the variance of this distribution then gives us some indication of the absolute loss of composite PDF descriptivity that occurs following feature-selection with respect to increasing dimensionality (since we have averaged over a full set of randomized morphologies). However, it is the loss of classification information with which we are most concerned.

The average misclassification rate with respect to full gamut of $i$ dimensional PDF morphologies under the tomographic scheme is, then, given as the integral: 


$$
\begin{array}{r}
\int_{X_{1}=X_{2}}^{X_{1}=\infty} \int_{X_{2}=0}^{X_{2}=\infty} \int_{P_{\text {tom. }}^{1}=P_{\text {tom. }}^{2}}^{P_{\text {tom. }}^{1}=\infty} \int_{P_{\text {tom. }}^{2}=0}^{P_{\text {tom. }}^{2}=\infty} X_{2} \cdot P\left(P_{\text {tom. }}^{1} \mid X_{1}\right) \cdot P\left(P_{\text {tom. }}^{2} \mid X_{2}\right) d P_{\text {tom. }}^{1} d P_{\text {tom. }}^{2} d X_{1} d X_{2} \\
+\int_{X_{2}=X_{1}}^{X_{2}=\infty} \int_{X_{1}=0}^{X_{1}=\infty} \int_{P_{\text {tom. }}^{2}=P_{\text {tom. }}^{1}}^{P_{\text {tom. }}^{2}=\infty} \int_{P_{\text {tom. }}^{1}=0}^{P_{\text {tom. }}^{1}=\infty} X_{1} \cdot P\left(P_{\text {tom. }}^{1} \mid X_{1}\right) \cdot P\left(P_{\text {tom. }}^{2} \mid X_{2}\right) d P_{\text {tom. }}^{1} d P_{\text {tom. }}^{2} d X_{1} d X_{2}
\end{array}
$$

where the sub/superscripts 1 and 2 indicate class labels.

That is, we implicitly sum over the two sets of possibilities for which class misattribution errors occur: $\left\{P_{\text {tom. }}^{1}>P_{\text {tom. }}^{2}\right.$ when $\left.X_{2}>X_{1}\right\}$ and $\left\{P_{\text {tom. }}^{2}>P_{\text {tom. }}^{1}\right.$ when $\left.X_{1}>X_{2}\right\}$. A numerically-computed graph of the outcome of this equation for a range of dimensionalities is given in figure 26 .

\subsection{Sum Rule Model Solution}

Turning now to the equivalent formulation for the sum rule combination scheme, the predicted value of the composite feature-space probability density at point $(1,1,1 \ldots)$ for a randomized morphology, $\left(P_{\text {sum }} \mid X\right)$, is given, for an underlying value $X$, by the formula:

$$
P_{\text {sum }} \mid X=\sum_{m=0}^{m=i}{ }^{m} A_{1} /\left(i 2^{i-1}\right)
$$

(we here introduce a normalization $\left(i 2^{i-1}\right)$ for consistency with the stochastic approach above).

The calculation of the way in which this quantity is distributed is complicated by the fact that many of the terms implicit in the individual summation, ${ }^{x} A_{1}$, are also implicit 
in a number of the other summations (specifically, at the various intersections of the hyper-planes represented by the ${ }^{x} A_{1}$ ). However, by explicitly acknowledging that each of the constituent hyper-planes essentially constitutes a sum over all of the points of the composite posterior PDF having coordinates with consecutive ordinates held at unity, we can isolate the various independent coordinate values in multiples:

$$
P\left(P_{\text {sum }} \mid \vec{X}\right)=\sum_{k=0}^{k=i}\left[k \sum_{m=1}^{m={ }^{i} C_{k}} X_{m}\right]
$$

(where the $X_{m}$ are independently selected from the distribution $\sqcap$ ).

The summation over every $X_{m}$ for a particular $k$ thus represents the set of $k$ coordinates having equal numbers of ordinals of value 1.

When the ensemble average is sought over every possible randomized morphological permutation of the composite prior pattern-space PDF in the previous manner, the predicted prior PDF value at $(1,1,1 \ldots)$ is thus distributed as:

$$
P(H \mid \vec{X})=\left(1(\sqcap \star)^{i} C_{1}\right) \star\left(2(\sqcap \star)^{i} C_{2}\right) \star\left(3(\sqcap \star)^{i} C_{3}\right) \star \ldots\left(i(\sqcap \star)^{i} C_{i}\right.
$$

The calculation of the misclassification rate is then achieved as before (equation 83), via an integration over every probability for which the predicted value of class 1 at $(1,1,1 \ldots), P_{\text {sum }}^{1}$, is of the opposing magnitude to the equivalent point of class $2, P_{\text {sum }}^{2}$, in relation to the actual value disparity: again see figure 26 for a numerical calculation of this quantity against dimensionality. 


\subsection{Product Rule Model Solution}

The calculation of misclassification rate with respect to randomized morphology for the product rule is considerably more involved in the previous cases as a consequence of the proliferation of terms with mixed products of higher variate powers as dimensionality

increases. As such, the misclassification rate verses dimensionality calculation may, very possibly, not be generally formalizable except on a case-by-case basis. A partial mathematical treatment may, however, be encompassed by approximation: that is, by explicitly assuming the independence of the summed terms in each ordinal projection from their counterparts in the remaining ordinal projections.

The probability density distribution of each ordinal is then as derived previously:

$$
P\left({ }^{x} A_{2}\right) d^{x} A_{2}=(\sqcap \star)^{2^{i-1}}
$$

However, it is the multiplicative value density of these terms with which we are primarily interested:

$$
P\left(P_{\text {prod. }} \mid X\right)=P\left({ }^{1} A_{1} *^{2} A_{1} * \ldots * *^{z} A_{1} \mid X\right)
$$

(overlooking normalization considerations).

Hence, we need to apply a logarithmic substitution in order to render the distribution tractable as a convolution of random variates:

$$
P\left(\log \left(P_{\text {prod. }}\right)\right)=P\left(\log \left({ }^{1} A_{1}\right)+\log \left({ }^{2} A_{1}\right)+\ldots \log \left({ }^{z} A_{1}\right)\right)
$$




$$
\left.\Rightarrow P\left(\log \left(P_{\text {prod. }}\right)\right)=P\left(\log \left({ }^{1} A_{1}\right)\right) \star P\left(\log \left({ }^{2} A_{1}\right)\right) \star \ldots P\left(\log \left({ }^{z} A_{1}\right)\right)\right)
$$

The distribution resulting from this substitution is thus approximately log-normal (increasingly so as dimensionality increases via the multiplicative central limit theorem): Montecarlo simulation for the lower end of the dimensional range tends to confirm the accuracy of the adopted approximation.

The performance results in terms of the correct class attribution rate for the product rule are as given in figure 26 via the formulation of equation 83, which, along with the previous results, thus serves as our baseline performance, 'noise response' model over the dimensional range: direct comparison with the results for the 'real-world' geological survey data given in figure 27 are thus invited. We re-emphasize in passing that the vertical ordinate of graph 27 represents the ensemble average error-rate: the error bars thus refer to the unbiased estimate of the standard deviation of the mean of the error rate (a figure which would otherwise be partially dictated by the number of samples, $={ }^{26} C_{i}$, contributing to each test point of the dimensional range. This consideration, however, does not supersede the fact of an inevitably incremental correlation among the individual samples as the dimensionality increases (in consequence of a greater degree of overlapping among the feature subsets), manifesting itself as a decreasing sample variance with increasing $i$ (without, in principle, affecting the mean to any great degree). Hence we opt to terminate the sequence at a figure significantly smaller than the total dimensionality to mitigate the impact of this effect.

We also note that in the wider interpretation of figures 26 and 27 , the horizontal graph axis could be equivalently labeled 'classifier number' rather then 'composite feature 
space dimensionality', the results being intended to be at least indicative of the more general fusion scenario for which classifiers are not necessarily limited to representing single features (via the argument of section 5.1).

\subsection{Findings of Dimensionality Tests}

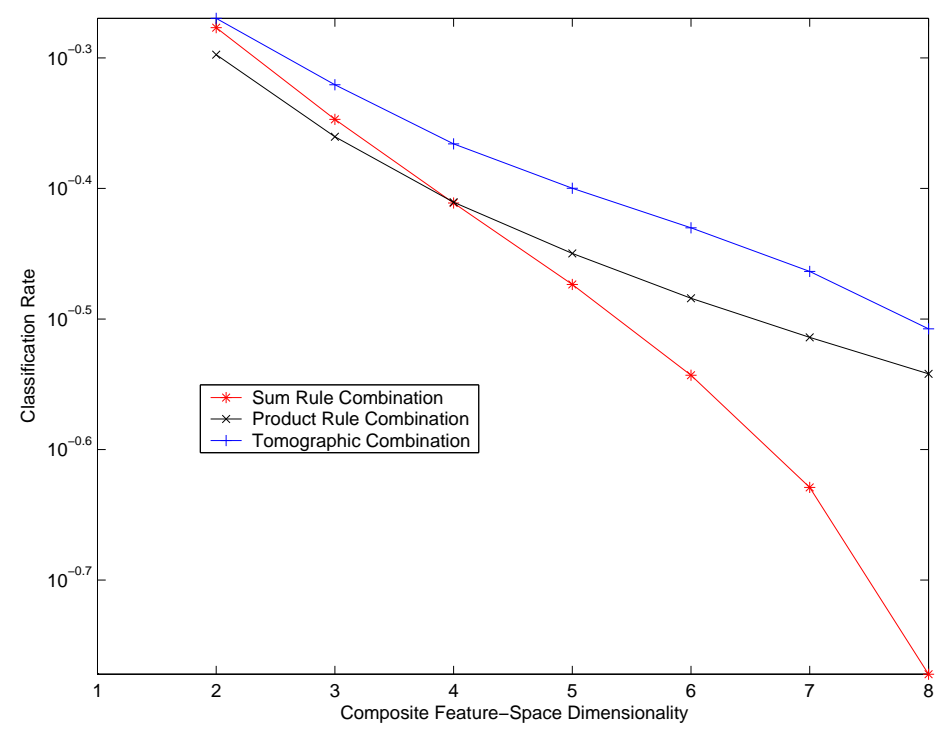

Figure 26: Classification Rate vs Dimensionality for the Model Data

In presenting an analysis of the results quantified in figures 26 and 27, the first point to notice (briefly alluded to earlier) is that, even though the tomographic method is disadvantaged by the specifically randomized nature of the morphology in the 'base-line' performance test (thereby imposing an absolute minimum of correlatable morphology between the various classifiers' PDFs), the performance graph of figure 26 suggests that it is, in terms of classification performance, nonetheless the superior combination methodology at every point of the dimensional range with respect to the sum and product rule alternatives. That is, the tomographic method, by virtue of making use of all of the data available in the classifiers constituting the combination (utilizing the cross- 


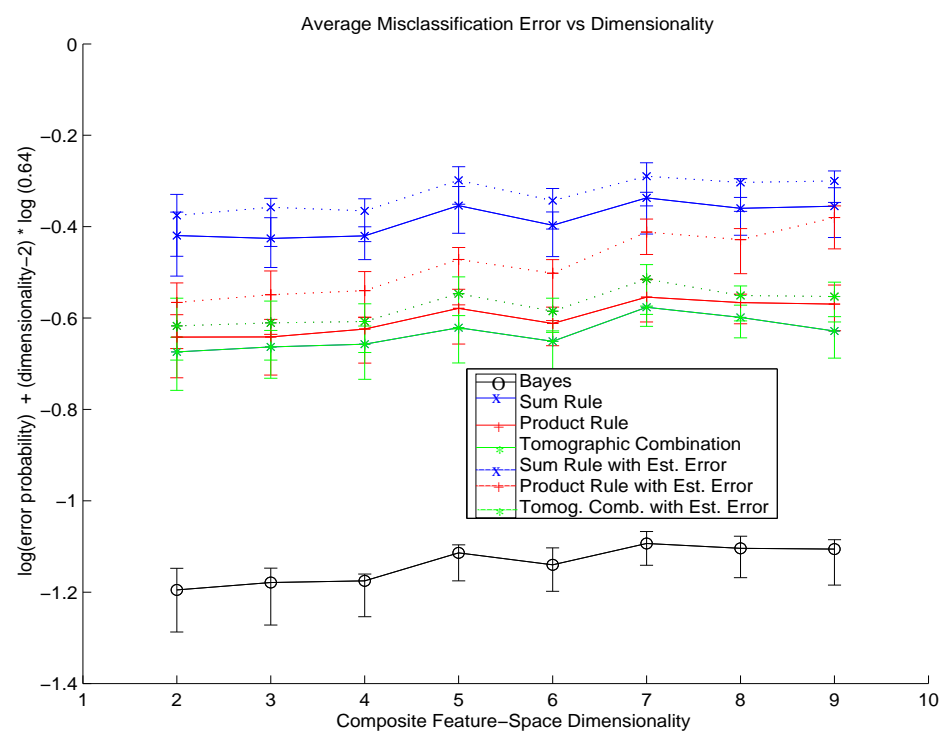

Figure 27: Misclassification Rate vs Dimensionality for the Real-World Data (note linearizion of vertical scale)

referenced information contained within every classifier ordinal, rather than just those constituting the implicitly reconstructed co-ordinate), is able to recover a greater extent of the composite pattern-space's PDF lost during the feature selection process than can existing methods (as represented by the sum and product rules via the argument of [2]).

The differences between the reconstructive abilities of the methods are encapsulated in the distributions $P\left(P_{\text {tom. }} \mid X\right), P\left(P_{\text {sum }} \mid X\right)$ and $P\left(P_{\text {prod. }} \mid X\right)$, describing the deviation from the prior composite probability density value at the implicitly reconstructed patternspace co-ordinate. That the performance results of the three methods are not especially different relative to the simulated Bayes error rate (which descends logarithmically) is thus speculated to be a consequence of the fact that the variances of these distributions are of similar orders of magnitude for constant dimensionalities, being governed chiefly by the number of self-convolutions of the uniform function, with higher numbers thus progressively approximating the Gaussian form (or log-Gaussian form, in the case of the product rule). This 'convolution number' being the same for the sum, product and 
tomographic combination methods means that the difference between the techniques, the 'methodological signature', as it were, is manifested solely by the differences in the shape of the distribution functions.

Thus, in retrospect, we can appreciate that any combination method postulating a composite PDF solution consistent with the ordinal projection values (as the sum, product and tomographic methods all do), will prove to give very similar classification accuracies when the class morphologies are randomized over all ensembles. It is, as stated earlier, only when correlated classifier morphologies can be related to each other that advantages of tomographic combination come significantly to light.

Turning then to the real-world performance tests, for which we postulated that correlated morphology among the classifiers is the rule rather that the exception, the equivalent results for the rock-strata data (figure 27) would seem to indicate that the ability of the tomographic combiner to correlate morphology between the $i$ discrete classifiers is much more in evidence, with a clear performance advantage over the sum and product rules developing with increasing dimensionality.

In terms of the point-by-point relationship between the three combining methodologies, it would appear that the tomographic method more closely mimics the performance of the product rule than the sum rule, despite its origins in the latter technique. We hypothesize that this is a consequence of actual independence in the original PDFs being recovered by the tomographic method (which is feasible, given that, on inspection, the prior PDFs have an approximately similar morphology to the Gaussian distribution of uniform covariance). It should be noted, however, that the tomographic estimationerror graph more closely parallels that of the sum rule than the product rule (as we would conceivably expect, given the results of 'base-line' performance measure tests of 
figure 26. Thus, in a sense, figure 26 and its attendant mathematical derivations can be considered to additionally serve as an indicator of the isolated effects of estimation error on the respective tomographic, sum and product combination rules (the estimation error plots in figure 27 then correspondingly being seen as contextual indicators of the effects of estimation error). That is, the point-by-point randomness of the prior PDFs in the model solution gives rise to a noise function at each of the classifier ordinates (the sum of the independent random variables being binomially distributed) that behaves similarly to the simulated estimation error of the second investigation, albeit without the context of the real-world classifier PDFs.

\subsection{Conclusions to Dimensionality Tests}

In terms of the advocation of a general combination strategy for an unfamiliar classification problem on the basis of the tests we have conducted, it would seem that the tomographic method is the indicated approach, both in terms of its reconstructive ability, as well as in its estimation error resilience (for which the method approaches the performance of the sum rule, without that technique's reconstructive deficiencies): in particular, these advantages would appear to scale favorably with the number of classifiers constituting the combination.

It must, however, be clearly understood that the scatter of data points in figure 27 is such that it is not possible to guarantee in all cases (or even much more than half of the cases for the lower dimensionalities) that the tomographic method is optimal (it being always possible to consider composite pattern-space PDF morphologies that favor either of the alternative strategies). Our argument, we emphasize, is with respect to arbitrary 
underlying PDF morphologies ${ }^{6}$, for which the presence of back-projection artifacts implied by conventional linear combination methods (the gamut of which the sum and product rules are deemed to collectively encompass) are taken to be generally unrepresentative. It is interesting to note, however, on the evidence of figure 27 , that in the real world scenario, despite the presumed presence of these artifacts, the product rule would appear to be significantly better at composite PDF morphology recovery than the sum rule. This is presumably a consequence of the fact that the reconstruction artifacts are suppressed (but, note, not fully removed) via repeated multiplication. This advantage, however, is generally suppressed by the multiplicative cumulation of estimation-error effects for all but ideal classifiers.

We have, thus, provided performance statistics to complement the earlier theoretical assertion that tomographic combination recovers the greatest degree of the composite pattern space PDF morphology lost during feature selection (the precise quantity of recoverable information being indicated by the disparity between the Bayesian and tomographic error rates for figures 26 and 27 with regard to artificial and real situations, respectively).

Moreover, we have demonstrated that the tomographic method, as well as having the best underlying performance rate, has also a similar error resilience to the sum-rule combination methodology, thereby combining the best of both of the aspects of combination through which classification performance is improved, the morphologically reconstructive and the error-negating: these two aspects being previously partially, though separately, represented within the product and sum rules, respectively.

\footnotetext{
${ }^{6}$ as distinct from the randomized morphologies of our model solution.
} 


\section{Morphology-Centered Classifier Combination: Ret-}

\section{rospect, Prospect}

We have, in this article, set out an analogy in which the range of classifier combination strategies represent, in so far as the feature sets are distinct, the incomplete tomographic reconstruction of the combined pattern-space probability density function from Radon transform data presented by the feature selection process. After accommodating the specific issues arising from the higher dimensionality and lower angular sample-rates of the Radon transforms within this regime, the metaphor immediately indicated a methodology for performance optimization through the application of the prefiltering convolution of equation 7 to the classifier PDFs prior to back-projection. We have thus achieved an optimization of classifier combination that proceeds from a priori grounds, rather than the more usual approach of optimizing from within a pre-existing combination strategy selected on contingent or heuristic grounds (cf eg [19, 20, 21, 22, 23]).

Our assertion of the morphological optimality of our method then centers on it being a full completion of the partial tomographic reconstruction process implicit in all conventional methods of classifier combination, the only other considerations that we need address in this regard being, firstly, that of the remaining aspect of combination as implicit refinement of the PDF morphologies and, secondly, the robustness of the reconstructive procedure in relation to estimation error. The former point is necessarily now addressed at the level of feature selection and hence, within our unifying perspective, may be carried out at an optimal level through having distinguished it from the purely tomographic aspects of classical combination. The latter concern, the robustness of the 
procedure to estimation error, is argued to be of the order of that of the Sum Rule, the previously optimal procedure in this regard, and although exact calculation was omitted due to the dependence of the filtering procedure on the nature of the input PDFs, we found evidence to support this claim in the practical and model-theoretical experiments of section 5 .

Another major area of concern, set out in detail in section 3.2, was to significantly reduce the computation time involved in tomographic fusion. By reinterpreting the methodology in graphical correlation terms, we were able to achieve a reduction of many orders of magnitude. This is sufficient that the method no longer poses any significant cost obstacle to the implementation of the procedure with current computer technology.

The exact basis of this efficiency gain was the appreciation that, when viewed in terms of the constituent PDFs, the three chief computational components within the recursive procedure (the peak-seek, the analysis of the correlation between detected peaks and the subtraction/registration of those correlated components) need not be performed on an individual basis, potentially reducing the iteration requirement from $X^{n}[X]^{n}\left[X^{n-1}+X\right]$ computational cycles to $X^{n}$ computational cycles, with the further possibility of an order of magnitude decrease in this figure for point-wise continuous classifiers. This is in addition to gains arising from the requirement that the $\delta z$ parameter effectively vary throughout the procedure.

It was further anticipated that this PDF-centered approach might ultimately lend itself to a future reinterpretation of the optimal methodology for multiple expert fusion without any explicit reference to tomography theory, being rendering instead in the more familiar terminology of probability theory.

Implementing, in section 5, this economized strategy on a set of practical and model 
scenarios over a range of dimensionalities, we argued that in terms of the advocation of a general combination strategy for an unfamiliar classification problem on the basis of the tests we have conducted, it would appear that the tomographic method is the indicated approach, both in terms of its reconstructive ability, as well as in its estimation error resilience (for which the method mimics the performance of the sum rule, without that technique's reconstructive deficiencies). In particular, these advantages would appear to scale favorably with the number of classifiers constituting the combination.

It should, however, be clearly understood in making this argument that the scatter of data points in Figure 27 is such that it is not possible to guarantee in all cases (or even much more than half of the cases for the lower dimensionalities) that the tomographic method is optimal (it being always possible to consider composite pattern-space PDF morphologies that favor either of the alternative strategies). Our argument, we emphasize, is with respect to arbitrary underlying PDF morphologies ${ }^{7}$, for which the presence of back-projection artifacts implied by conventional linear combination methods (the gamut of which the sum and product rules are deemed to collectively encompass) are taken to be generally unrepresentative. It is interesting to note, however, on the evidence of Figure 27, that in the real world scenario, despite the presumed presence of these artifacts, the product rule would appear to be significantly better at composite PDF morphology recovery than the sum rule. This is presumably a consequence of the fact that the reconstruction artifacts are suppressed (but, note, not fully removed) via repeated multiplication. This advantage, however, is generally suppressed by the multiplicative cumulation of estimation-error effects for all but ideal classifiers.

\footnotetext{
${ }^{7}$ as distinct from the randomized morphologies of our model solution.
} 


\subsection{Outlook}

With respect to the prospects for further improving the tomographic combination methodology, one possibility is to note that the modified Högbom method specified in section 3.4.3 inherently regards the rectanguloid hypercube as its deconvolution primitive, and thus constitutes only a partial realization of the potential for applying tomographic filtration to combined classifiers (the central idea of which is removal of all axial bias from back-projected radon data). Clearly, while the rectanguloid hypercube primitive serves to remove much of the the feature axial alignment imposed by classifier combination (in particular, the elongated striations depicted in Figure 14), it still exhibits an obvious axial alignment on the local scale. Thus there is scope for future methodological improvement by introducing more rotationally symmetric primitives (for instance hyper-ovoids, which would be capable of reconstructing complete Gaussians).

Another, complementary, approach is to seek to increase the computational performance of the tomographic method, which at present, though considerably economized [18] (and parametrically tunable to a high degree), falls significantly behind the linear sum and product methods. To remedy this situation, it is necessary to employ a pre-filtration approach. That is, we should have to apply a filtering convolution to the individual classifier PDFs and combine via the sum rule, imposing a positivity condition on the output. Such a method, while conjectured to be of somewhat less accuracy than the current approach, would have the benefit of scaling linearly in terms of operation time with the number of classifiers. To determine exactly what the accuracy deficit might be for such a procedure would be the basis of further empirical and theoretical investigation.

It might also be of interest to consider alongside an investigation of this type the suitabil- 
ity of differing base classifiers as candidates for pre-filtration. In principle our method is completely independent with regard to underlying base classifier type (as argued in 2.1 on a theoretical level, an also experimentally in section 4 in relation to $\mathrm{NN}$, Quadratic and Gaussian classifiers in various combinations). However, the practical pay-off in each case might not be justified; efficient pre-filtration prior to tomographic reconstruction requires that there exists a mathematical representation of the base classifier PDFs amenable to convolution (such as could, for instance, be determined even for non-Bayesian classifiers like decision trees by the explicit elaboration of their recursive division of the feature space into hyperplane sections). If such a direct formulation is not straightforwardly available, it would be necessary to construct it artificially via sampling and interpolation; in itself a form of PDF estimation, and thus prone to an additional source of estimation error. In some cases one may therefore be justified in compromising accuracy in the interests of reducing computation time. Equally, one may prefer handling combination in a manner conceptually congruent with the underlying base classifier paradigm (for instance, employing a neural net combination layer for combining neural nets). However, while morphologically consistent, such approaches (unlike tomographic combination) can never be considered morphologically unbiased.

In conclusion to this article, then, we have undertaken a series of experimental investigations to demonstrate the utility of our theoretical understanding that tomographic combination methodologies have the capability to reconstruct the composite pattern space PDF morphology lost during feature selection in the most morphological unbiased fashion. Classifiers in this scenario can hence be considered to act as 'morphology probes' within the context of the feature selection process, classifier morphology being matched to training data morphology as appropriate throughout the procedure. The 
method thus exists at a meta-level to both classification and (via the argument of section 2.7) classifier combination in its conventional form, and hence should not be invalidated by future developments in the field (in particular new forms of classification; even though a particular classifier might arise which is capable of representing the composite pattern space without any reconstructive loss, that classifier is immediately appended to the range of possible morphology descriptors available to the optimal feature selection mechanism of section 2.7).

Furthermore, we have demonstrated that the procedure has an error resilience comparable to that of the sum-rule combination methodology, thereby combining both of the aspects of combination through which classification performance is improved, the morphologically reconstructive and the error-negating.

\section{Acknowledgment}

This research was carried out at the University of Surrey, UK, supported by, and within the framework of, EPSRC research grant number GR/M61320.

Particular thanks are due to Josef Kittler for his unstinting technical and practical guidance throughout the long gestation and eventual fruition of this project.

Personal thanks are due to my wife Andrea for her equally unwaivering emotional support throughout. 


\section{References}

[1] R A Jacobs, "Methods for combining experts' probability assessments", Neural Computation, 3, pp 79-87, 1991

[2] J. Kittler, M. Hatef, R.P.W. Duin, and J. Matas, "On combining classifiers", IEEE Transactions on Pattern Analysis and Machine Intelligence, vol. 20, no. 3, 1998, $226-239$

[3] L. Lam and C.Y. Suen, "Optimal combinations of pattern classifiers", Pattern Recognition Letters, vol. 16, no. 9, 1995, 945-954.

[4] A F R Rahman and M C Fairhurst, "An evaluation of multi-expert configurations for the recognition of handwritten numerals", Pattern Recognition Letters, 31, pp $1255-1273,1998$

[5] A F R Rahman and M C Fairhurst, "A new hybrid approach in combining multiple experts to recognise handwritten numerals", Pattern Recognition Letters, 18, pp 781-790, 1997

[6] K Woods, W P Kegelmeyer and K Bowyer, "Combination of multiple classifiers using local accuracy estimates", IEEE Trans. Pattern Analysis and Machine Intelligence, 19, pp 405-410, 1997

[7] D. Windridge, J. Kittler, "A Generalised Solution to the Problem of Multiple Expert Fusion.", (Univ. of Surrey Technical Report: VSSP-TR-5/2000)

[8] F. Natterer, Proceedings "State of the Art in Numerical Analysis", York, April1-4, 1996. 
[9] J. Högbom, "Aperture synthesis with a non-regular distribution of interferometer baselines", Astrophys. J. Suppl. Ser., 15, 417-426, 1974

[10] Briggs, D. S.; Cornwell, T. J. "An Alternative Interpretation for the Physical Basis of CLEAN", Astronomical Data Analysis Software and Systems I, A.S.P. Conference Series, Vol. 25, 1992, Eds Diana M. Worrall, Chris Biemesderfer, and Jeannette Barnes, eds., p. 170.

[11] D. Windridge, J. Kittler, "Combined Classifier Optimisation via Feature Selection", Advances in Pattern Recognition, LNCS. Vol. 1876, August 2000.

[12] K. M. Ali and M.J. Pazzani, "On the link between error correlation and error reduction in decision tree ensembles", Technical Report 95-38, ICS-UCI, 1995

[13] T.K. Ho, J.J. Hull, and S.N. Srihari, "Decision combination in multiple classifier systems", IEEE Transactions on Pattern Analysis and Machine Intelligence, vol. 16, no. $1,1994,66-75$.

[14] L. Xu, A. Krzyzak and C.Y. Suen, "Methods of combining multiple classifiers and their application to handwriting recognition", IEEE Trans. SMC, vol. 22, no. 11, $1994,1539-1549$

[15] J. Kittler, "Improving Recognition Rates by Classifier Combination: A Review", 1st IAPR TC1 Workshop on Statistical Techniques in Pattern Recognition, 205-210, June 1997

[16] Cornwell, T. J. \& Evans, K. F. (1985), “A simple maximum entropy deconvolution algorithm", Astron. Astrophys., 143, 77-83. 
[17] D. Windridge, J. Kittler, "A Morphologically Optimal Strategy for Classifier Combination: Multiple Expert Fusion as a Tomographic Process", IEEE PAMI, Vol 25, no. 3, March 2003

[18] D. Windridge, J. Kittler, Economic Tomographic Classifier Fusion: Eliminating Redundant Högbom Deconvolution Cycles in the Sum-Rule Domain (Univ. of Surrey Technical Report: VSSP-TR-1/2003)

[19] Intrator N. and Cohen S., "Automatic model selection in a hybrid perceptron/radial network", Information Fusion 3 (2002); 259-266

[20] Breiman L., "Bagging predictors", Journal of Machine Learning, 1996; vol. 24, no. $2: 123-140$.

[21] Drucker H., Cortes C., Jackel L. D., Lecun Y., and Vapnik V., Boosting and other ensemble methods, Neural Computation, 1994; vol. 6, no. 6:1289-1301

[22] Bruzzone L., Cossu R. and Vernazza G., "Combining parametric and nonparametric algorithms for a partially unsupervised classification of multitemporal remote-sensing images", Information Fusion 3 (2002); 289-297

[23] Dietterich T. G., Bakiri G. "Solving Multiclass Learning Problems via ErrorCorrecting Output Codes." Journal of Artificial Intelligence Research, 1995; Vol 2.: $263-286$.

[24] Breiman L. "Bagging predictors", Journal of Machine Learning, 1996; 24(2):123140

[25] Freund Y, Schapire RE. Experiments with a new boosting algorithm. Proceedings 13th International Conference on Machine Learning 1996; 148-156 
[26] Ho TK. The Random subspace method for constructing decision forests. IEEE Trans Pattern Analysis and Machine Intelligence 1998; 20(8): 832-844

[27] P. Melville, N. Shah, L. Mihalkova, R.J. Mooney, Experiments on Ensembles with Missing and Noisy Data, LNCS, Vol. 3077, June 2004. 